Supporting Information for:

\title{
Dearomative Synthetic Entry Into the Altemicidin Alkaloids
}

\author{
Claire S. Harmange Magnani and Thomas J. Maimone* \\ Department of Chemistry, University of California-Berkeley, 826 Latimer Hall, Berkeley, CA, \\ 94720 \\ *maimone@berkeley.edu
}

General Procedures $\quad 2$

Compound Preparation and Characterization Data 4

Natural Product Spectral Comparisons $\quad 30$

ESP Surface Data $\quad 32$

References $\quad 35$

Appendix A: Ammonia Methanol Solution 36

$\begin{array}{ll}\text { Appendix B: NMR Spectra } & 37\end{array}$ 


\section{General Procedures}

All reactions were performed in flame- or oven-dried glassware under positive pressure of nitrogen or argon, unless otherwise noted. Air- and moisture-sensitive liquids were transferred via syringe. When indicated, solvents or reagents were degassed by sparging with argon for 10 minutes in an ultrasound bath at $25{ }^{\circ} \mathrm{C}$. Volatile solvents were removed under reduced pressure rotary evaporation. Analytical and preparative thin-layer chromatography (TLC) were performed using glass plates coated with silica gel $(0.25-\mathrm{mm}, 60-\AA$ pore size, Merck TLC Silicagel 60 F254) impregnated with a fluorescent indicator $(254 \mathrm{~nm})$. TLC plates were visualized by exposure to ultraviolet light (UV) and then were stained by submersion in an ethanolic anisaldehyde solution, potassium permanganate solution, or ninhydrin solution followed by brief heating on a hot plate. Flash column chromatography was performed with silica gel purchased from Silicycle (SiliaFlash ${ }^{\circledR}, 60 \AA, 230-40$ mesh, 40-63 $\mu \mathrm{M}$ ). Reverse phase C18 silica was purchased from Acros Organics (C18-RP, 10\%C. ca $0.6 \mathrm{mmol} / \mathrm{g}$, part size $40-63 \mu \mathrm{M})$. All samples, unless otherwise noted, were dry loaded onto the column either by resuspension of the crude mixture in $\mathrm{Et}_{2} \mathrm{O}$, acetone, or $\mathrm{MeOH}$ with an appropriate amount of silica gel and careful concentration to dryness in vacuo. $7 \mathrm{~N} \mathrm{NH}_{3}$ in $\mathrm{MeOH}$ solution for TLC and column chromatography was made according to Appendix A: Ammonia Methanol Solution. Triethylamine, chlorotrimethylsilane, phenyl chloroformate, DBU, and acetyl chloride were distilled over calcium hydride prior to use. All other reagents were used as received from commercial sources, unless otherwise noted. Anhydrous acetonitrile $(\mathrm{MeCN})$, dichloromethane (DCM), tetrahydrofuran (THF), and dimethylformamide (DMF) were obtained by passing these previously degassed solvents through activated alumina columns. Anhydrous methanol $(\mathrm{MeOH})$, trifluorotoluene $\left(\mathrm{CF}_{3} \mathrm{Ph}\right)$, 1,4-dioxane, ethanol $(\mathrm{EtOH})$, and pyridine were purchased from Sigma Aldrich. Proton nuclear magnetic resonance $\left({ }^{1} \mathrm{H}\right.$ NMR $)$ 
spectra and carbon nuclear magnetic resonance $\left({ }^{13} \mathrm{C} N M R\right)$ spectra were recorded on Bruker AV300, AV500, AV600, and AV700 spectrometers at $23{ }^{\circ} \mathrm{C}$. Proton chemical shifts are expressed as parts per million (ppm, $\delta$ scale) and are referenced to residual solvent $\left(\mathrm{CHCl}_{3}, \delta 7.26(\mathrm{~s}), \mathrm{C}_{6} \mathrm{DH}_{5}\right.$, $\left.\delta 7.16(\mathrm{~s}), \mathrm{CD}_{2} \mathrm{HOD} \delta 3.31(\mathrm{p}), 4.87(\mathrm{~s}, \mathrm{DOH}), \mathrm{DOH} \delta 4.80(\mathrm{~s})\right)$. Carbon chemical shifts are expressed as parts per million (ppm, $\delta$ scale) and are referenced to the solvent $\left(\mathrm{CDCl}_{3}, \delta 77.16\right.$, $\left.\mathrm{C}_{6} \mathrm{D}_{6}, \delta 128.1, \mathrm{CD}_{3} \mathrm{OD} \delta 49.00\right)$. Data is presented as follows: chemical shift, multiplicity (s $=$ singlet, $\mathrm{d}=$ doublet, $\mathrm{dd}=$ doublet of doublets, $\mathrm{ddd}=$ doublet of doublet of doublet, $\mathrm{dt}=$ triplet of doublet, $\mathrm{t}=$ triplet, $\mathrm{q}=$ quartet, $\mathrm{m}=$ multiplet, $\mathrm{br}=$ broad, coupling constant $(J)$ in $\operatorname{Hertz}(\mathrm{Hz})$, and integration. Infrared (IR) spectra were recorded on a Bruker Alpha FT - IR spectrometer as thin films and are reported in frequency of absorption $\left(\mathrm{cm}^{-1}\right)$. Only selected resonances are reported. High-resolution mass spectra (HRMS) were obtained by the mass spectrometry facility at the University of California Berkeley using a Finnigan LTQFT mass spectrometer (Thermo Electron Corporation). X-ray diffraction data was collected at the Small Molecule X-ray Crystallography Facility (CheXray) at the University of California, Berkeley using a Rigaku XtaLAB P200 equipped with a MicroMax 007HF rotating anoted an Pilatus3 R 200K-A hybrid pixel array detector. Data were collected using CuKa radiation $(1=1.5418 \AA)$. 


\section{Compound Preparation and Characterization Data}
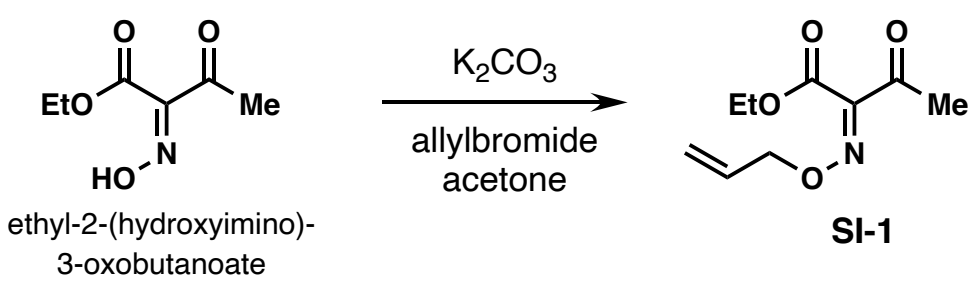

Allyl oxime SI-1: To a solution of ethyl-2-(hydroxyimino)-3-oxobutanoate (44.0 g, $276.6 \mathrm{mmol}$, 1.0 equiv. $)$ in acetone $(300 \mathrm{~mL})$ was added potassium carbonate $(57.3 \mathrm{~g}, 414.9 \mathrm{mmol}, 1.5 \mathrm{equiv}$.) followed by allyl bromide (35.9 mL, $414.9 \mathrm{mmol}, 1.5$ equiv.) at room temperature. Vigorous stirring was maintained for 45 minutes or until TLC showed consumption of starting material. The reaction mixture was then filtered through a pad of Celite ${ }^{\circledR}$, concentrated, and purified by silica flash column chromatography $\left(5,10,15 \% \mathrm{Et}_{2} \mathrm{O} / \mathrm{Hexanes}\right)$ to yield allyl oxime SI-1 (45.5 g, 228.5 mmol, $83 \%$ yield) after concentrating three times with toluene and drying overnight on a vacuum line to remove residual water.

TLC: $\mathrm{R}_{\mathrm{f}}=0.57$ (25\% EtOAc in hexanes).

${ }^{1} \mathbf{H}$ NMR $\left(700 \mathrm{MHz}, \mathrm{CDCl}_{3}\right) \delta 5.97(\mathrm{ddt}, J=17.3,10.5,5.7 \mathrm{~Hz}, 1 \mathrm{H}), 5.34(\mathrm{dd}, J=17.3,1.6 \mathrm{~Hz}$, 1H), $5.28(\mathrm{dd}, J=10.5,1.6 \mathrm{~Hz}, 1 \mathrm{H}), 4.77(\mathrm{~d}, J=5.7 \mathrm{~Hz}, 2 \mathrm{H}), 4.35$ (q, $J=7.1 \mathrm{~Hz}, 2 \mathrm{H}), 2.39$ (s, $3 \mathrm{H}), 1.33(\mathrm{t}, J=7.2 \mathrm{~Hz}, 3 \mathrm{H})$.

${ }^{13}$ C NMR $\left(176 \mathrm{MHz}, \mathrm{CDCl}_{3}\right) \delta 193.1,161.3,150.5,132.4,119.2,77.6,77.3,77.2,77.0,62.2$, $25.3,14.2$.

HRMS (m/z): (ESI) calcd. for $\mathrm{C}_{19} \mathrm{H}_{13} \mathrm{NO}_{4} \mathrm{Na}[\mathrm{M}+\mathrm{Na}]^{+}:$222.0737, found 222.0738.

IR (thin film) $v_{\max }\left(\mathbf{c m}^{-1}\right):$ 2984, 1744, 1698, 1369, 1234, 1009. 


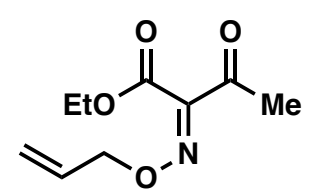

SI-1

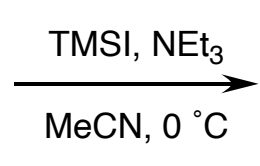

\section{,}

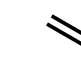

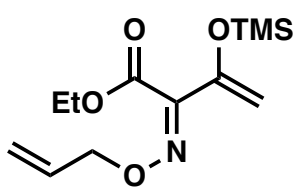

6

Silyl enol ether 6: To a 500-mL round bottom flask containing SI-1 (22.1 g, 110.9 mmol, 1.0 equiv.) and sodium iodide (18.3g, $122.0 \mathrm{mmol}, 1.1$ equiv.) was added MeCN (139 mL). The mixture was cooled to $0{ }^{\circ} \mathrm{C}$ and stirred vigorously until dissolution of the sodium iodide. To the cooled solution was added triethylamine $(61.8 \mathrm{~mL}, 443.8 \mathrm{mmol}, 4.0$ equiv.) followed by chlorotrimethylsilane $(21.1 \mathrm{~mL}, 166.4 \mathrm{mmol}, 1.5$ equiv.). The resulting suspension was removed from the cooling bath and warmed to room temperature. Upon consumption of starting material as indicated by ${ }^{1} \mathrm{H}$ NMR of an aliquot, the reaction mixture was concentrated in vacuo to a brown solid, which was resuspended in 3:1 Et $2 \mathrm{O}:$ Hexanes, filtered through Celite ${ }^{\circledR}$, and concentrated in vacuo. The resulting brown oil was resuspended in copious hexanes, filtered through Celite ${ }^{\circledR}$, and concentrated in vacuo to afford silyl enol ether 6 (30.1 g, 110.9 mmol, quant. yield) as a yellow oil.

TLC: not stable to silica.

${ }^{1}$ H NMR $\left(300 \mathrm{MHz}, \mathrm{C}_{6} \mathrm{D}_{6}\right) \delta 5.78(\mathrm{ddt}, J=17.2,10.7,5.4 \mathrm{~Hz}, 1 \mathrm{H}), 5.16(\mathrm{dq}, J=17.2,1.6 \mathrm{~Hz}$, 1H), $4.98(\mathrm{dq}, J=10.7,1.4 \mathrm{~Hz}, 1 \mathrm{H}), 4.89(\mathrm{~d}, J=1.9 \mathrm{~Hz}, 1 \mathrm{H}), 4.66(\mathrm{~d}, J=1.9 \mathrm{~Hz}, 1 \mathrm{H}), 4.49(\mathrm{dt}, J$ $=5.4,1.6 \mathrm{~Hz}, 2 \mathrm{H}), 4.07(\mathrm{q}, J=7.1 \mathrm{~Hz}, 2 \mathrm{H}), 0.95(\mathrm{t}, J=7.1 \mathrm{~Hz}, 3 \mathrm{H}), 0.19(\mathrm{~s}, 9 \mathrm{H})$.

${ }^{13}$ C NMR (151 MHz, $\left.\mathrm{C}_{6} \mathrm{D}_{6}\right) \delta 163.0,150.8,149.3,134.0,117.6,100.2,76.1,61.5,14.1,0.2$.

IR (thin film) $\boldsymbol{v}_{\max }\left(\mathbf{c m}^{-1}\right): 2961(\mathrm{w}), 1739$ (s), 1613 (m), 1371 (m), 1350 (m), 1323 (s), 1182 (s), $1015(\mathrm{~s}), 843$ (s).

HRMS (m/z): (ESI) calcd. for $\mathrm{C}_{12} \mathrm{H}_{22} \mathrm{NO}_{4} \mathrm{Si}[\mathrm{M}+\mathrm{H}]^{+}:$: 272.1313, found 272.1314. 

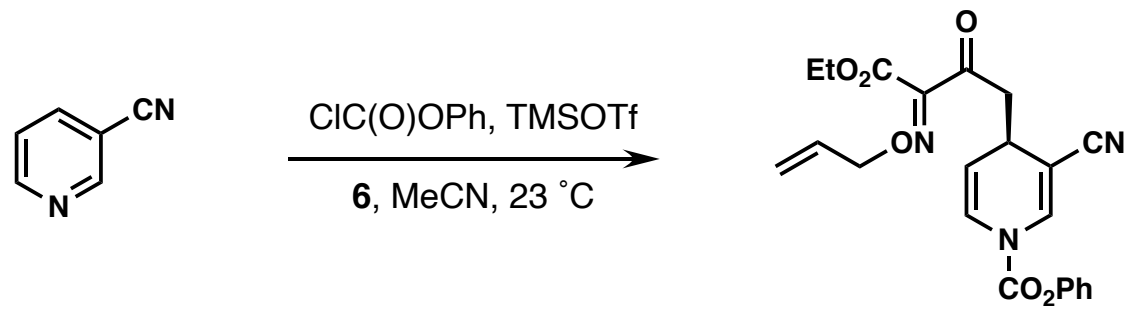

SI-2

Dehydropyridine SI-2: To a 1-L round bottom flask containing 3-cyanopyridine (1.9 g, 18.2 mmol, 1.0 equiv) was added $\mathrm{MeCN}(80 \mathrm{~mL})$ followed by phenyl chloroformate $(3.0 \mathrm{~mL}, 23.4$ mmol, 1.3 equiv.) and TMSOTf ( $0.350 \mathrm{~mL}, 1.8 \mathrm{mmol}, 0.10$ equiv.). After 15 minutes, silyl enol ether 6 (9.8 g, 36.1 mmol, 1.5 equiv.) was added dropwise via cannula as a solution in MeCN (80 $\mathrm{mL})$, and the flask was washed with additional $\mathrm{MeCN}(20 \mathrm{~mL})$, which was added to the reaction mixture. Upon consumption of 3-cyanopyridine, as indicated by TLC, the reaction mixture was quenched by cannulation into vigorously stirring $a q . \mathrm{NaOH}(1 \mathrm{M}, 300 \mathrm{~mL})$ at $0{ }^{\circ} \mathrm{C}$. The biphasic mixture was warmed to room temperature, poured into a separatory funnel, and extracted with EtOAc $(3 \times 200 \mathrm{~mL})$. The combined organic layers were washed with $a q . \mathrm{NaHCO}_{3}(1 \times 100 \mathrm{~mL})$, brine $(1 \times 100 \mathrm{~mL})$, dried over $\mathrm{Na}_{2} \mathrm{SO}_{4}$, and concentrated in vacuo. The orange oil was purified by column chromatography $(3,4,5,6 \%$ Et2O in $3: 1$ hexanes/DCM) to give the desired C-4 dehydropyridine adduct SI-2 (5.91 g, $14.0 \mathrm{mmol}, 77 \%$ yield) as a yellow oil.

TLC: $\mathrm{R}_{\mathrm{f}}=0.43(25 \%$ EtOAc in hexanes).

${ }^{1}$ H NMR $(600 \mathrm{MHz}, \mathrm{MeOD}) \delta 7.76(\mathrm{~s}, 1 \mathrm{H}), 7.43(\mathrm{dd}, J=8.6,7.3 \mathrm{~Hz}, 2 \mathrm{H}), 7.30(\mathrm{t}, J=7.4 \mathrm{~Hz}$, 1H), $7.23(\mathrm{~d}, J=7.4 \mathrm{~Hz}, 2 \mathrm{H}), 7.02-6.89(\mathrm{~m}, 1 \mathrm{H}), 6.03(\mathrm{ddt}, J=17.3,10.5,5.8 \mathrm{~Hz}, 1 \mathrm{H}), 5.38(\mathrm{dd}$, $J=17.3,1.6 \mathrm{~Hz}, 1 \mathrm{H}), 5.31(\mathrm{dd}, J=10.5,1.4 \mathrm{~Hz}, 1 \mathrm{H}), 5.19(\mathrm{dd}, J=8.1,4.5 \mathrm{~Hz}, 1 \mathrm{H}), 4.86-4.82$ (m, 2H), $4.33(\mathrm{q}, J=7.1 \mathrm{~Hz}, 2 \mathrm{H}), 3.71(\mathrm{dt}, J=7.9,4.5 \mathrm{~Hz}, 1 \mathrm{H}), 3.20(\mathrm{dd}, J=16.5,4.4 \mathrm{~Hz}, 1 \mathrm{H})$, $3.11(\mathrm{dd}, J=16.5,7.9 \mathrm{~Hz}, 1 \mathrm{H}), 1.31(\mathrm{t}, J=7.1 \mathrm{~Hz}, 3 \mathrm{H})$. 
${ }^{13}$ C NMR (151 MHz, MeOD) $\delta$ 193.6, 162.3, 151.9, 151.5, 150.3 (br), 137.6, 133.8, 130.6, 127.6, $123.3,122.5,119.6,118.9,110.6(\mathrm{br}), 94.3$ (br), 78.8, 63.2, 45.0, 31.5, 14.4.

IR (thin film) $\boldsymbol{v}_{\max }\left(\mathbf{c m}^{-1}\right): 2926,2213,1745,1688,1322,1202,1013$.

HRMS (m/z): (ESI) calcd. for $\mathrm{C}_{22} \mathrm{H}_{21} \mathrm{~N}_{3} \mathrm{O}_{6} \mathrm{Na}[\mathrm{M}+\mathrm{Na}]^{+}:$446.1323, found 446.1318.

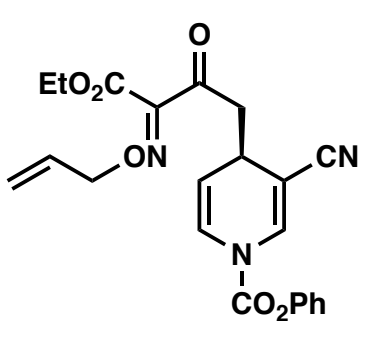

SI-2

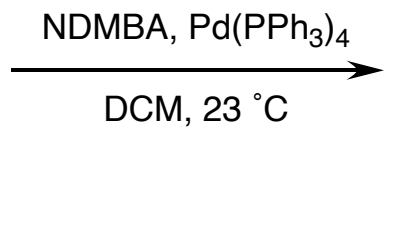

DCM, $23^{\circ} \mathrm{C}$

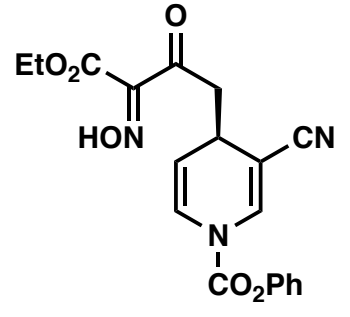

7

De-allyl dehydropyridine 7: 1,3-Dimethylbarbituric acid (NDMBA, $3.04 \mathrm{~g}, 19.5 \mathrm{mmol}, 0.50$ equiv.) and SI-2 (16.2g, $38.2 \mathrm{mmol}, 1.0$ equiv.) were loaded into a 500-mL round bottom flask. DCM (382 mL) was added, and the mixture was degassed. To this mixture was added palladiumtetrakis(triphenylphosphine) (441 $\mathrm{mg}, 0.382 \mathrm{mmol}, 0.01$ equiv.). Upon consumption of dehydropyridine $\mathrm{XX}$ as indicated by TLC, the reaction was quenched by addition of $\mathrm{Et}_{2} \mathrm{O}(20 \mathrm{~mL})$, followed by $p$-nitrobenzaldehyde $\left(3.00 \mathrm{~g}, 19.9 \mathrm{mmol}, 0.52\right.$ equiv.) and $\mathrm{H}_{2} \mathrm{O}(10 \mathrm{~mL})$ to consume excess NDMBA and ease purification. After stirring for 30 minutes, sat. aq. $\mathrm{NH}_{4} \mathrm{Cl}(300 \mathrm{~mL})$ was added and the resulting biphasic mixture was transferred to a separatory funnel, extracted with EtOAc $(3 \times 300 \mathrm{~mL})$, washed with brine $(1 \times 100 \mathrm{~mL})$, dried over $\mathrm{Na}_{2} \mathrm{SO}_{4}$, and concentrated in vacuo. The resulting brown oil was purified by column chromatography $\left(6,8,10 \% \mathrm{Et}_{2} \mathrm{O}\right.$ in $3: 1$ hexanes/DCM, then 20\% $\mathrm{Et}_{2} \mathrm{O}$ in 3:1 hexanes/DCM) to give 7 (13.5 g, $35.1 \mathrm{mmol}, 92 \%$ yield) as a pale yellow foam, which was dried overnight under hi-vac.

TLC: $R_{\mathrm{f}}=0.46(1: 1$ EtOAc in hexanes). 
${ }^{1}$ H NMR (300 MHz, MeOD) $\delta 7.71(\mathrm{~s}, 1 \mathrm{H}), 7.43-7.34(\mathrm{~m}, 2 \mathrm{H}), 7.29-7.22(\mathrm{~m}, 1 \mathrm{H}), 7.22-7.14$ $(\mathrm{m}, 2 \mathrm{H}), 6.90(\mathrm{~s}, 1 \mathrm{H}), 5.14(\mathrm{dd}, J=8.7,4.0 \mathrm{~Hz}, 1 \mathrm{H}), 4.28(\mathrm{q}, J=7.1 \mathrm{~Hz}, 2 \mathrm{H}), 3.66(\mathrm{dt}, J=7.4,3.7$ $\mathrm{Hz}, 1 \mathrm{H}), 3.27$ (p, $J=1.6 \mathrm{~Hz}, 1 \mathrm{H}), 3.19(\mathrm{dd}, J=16.8,4.4 \mathrm{~Hz}, 1 \mathrm{H}), 3.05(\mathrm{dd}, J=16.8,8.4 \mathrm{~Hz}, 1 \mathrm{H})$, $1.28(\mathrm{t}, J=7.1 \mathrm{~Hz}, 3 \mathrm{H})$.

${ }^{13}$ C NMR (151 MHz, MeOD) $\delta 194.6,163.4,152.2,151.9,150.3,137.5,130.6,127.5,123.2$, $122.5,118.9,110.7,94.4,62.9,44.9,31.3,14.4$.

IR (thin film) $v_{\max }\left(\mathbf{c m}^{-1}\right)$ : 3290 (br), 2981 (w), 2925 (w), $2856(\mathrm{w}), 2216$ (m), 2216 (m), 1742 (s), $1687(\mathrm{~s}), 1321(\mathrm{~s}), 1199(\mathrm{~s}), 1021(\mathrm{~m})$.

HRMS (m/z): (ESI) calcd. for $\mathrm{C}_{19} \mathrm{H}_{17} \mathrm{~N}_{3} \mathrm{O}_{6} \mathrm{Na}[\mathrm{M}+\mathrm{Na}]^{+}:$406.1010, found 406.1007.

(E)-oxime isomer (SI-3): (isolated $\sim 5 \%$ yield)

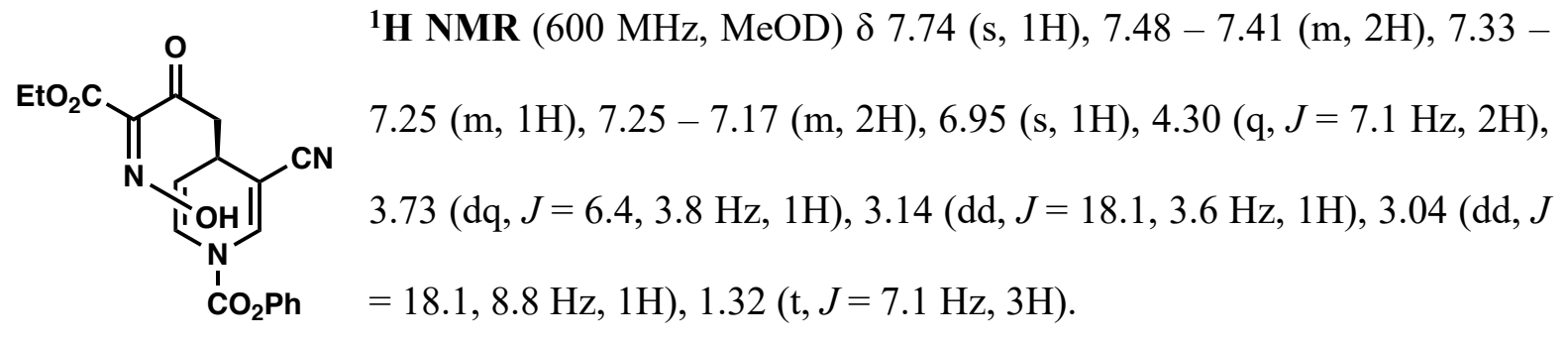

SI-3

TLC: $\mathrm{R}_{\mathrm{f}}=0.3$ (1:1 EtOAc in hexanes). 


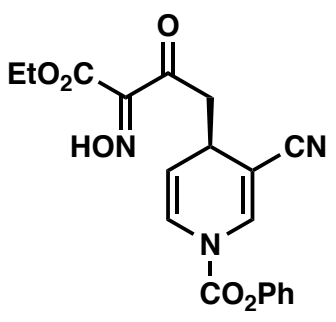

7

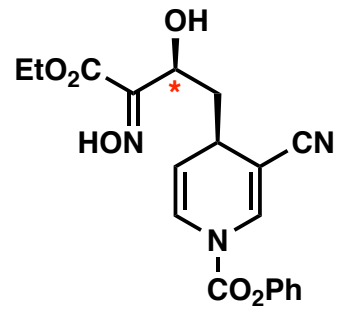

d.r. $1.7: 1$

8

Alcohol 8: To a 2-L round bottom flask containing 7 (7.88 g, $20.6 \mathrm{mmol}, 1.0$ equiv.) was added THF $(1.0 \mathrm{~L})$ and $\mathrm{MeOH}(20.6 \mathrm{~mL})$. The solution was cooled to $-40{ }^{\circ} \mathrm{C}$ in a dry ice-acetone bath equipped with a thermometer. Next, the rubber septum and nitrogen inlet were briefly removed to add sodium borohydride $(3.11 \mathrm{~g}, 82.2 \mathrm{mmol}, 4.0$ equiv) in a single portion. The reaction mixture was kept between $-40{ }^{\circ} \mathrm{C}$ and $-30{ }^{\circ} \mathrm{C}$ until consumption of 7 , as indicated by TLC. The reaction mixture was quenched by careful addition of $a q . \mathrm{HCl}(1 \mathrm{M}, 103 \mathrm{~mL})$ at $-40{ }^{\circ} \mathrm{C}$. The biphasic mixture was warmed to room temperature, transferred to a separatory funnel, extracted with EtOAc (3 x $300 \mathrm{~mL})$, washed with sat. aq. $\mathrm{NaHCO}_{3}(1 \times 300 \mathrm{~mL})$ and brine $(1 \times 300 \mathrm{~mL})$, dried with $\mathrm{Na}_{2} \mathrm{SO}_{4}$, and concentrated in vacuo. The resulting orange oil was purified by column chromatography (20\% EtOAc in hexanes $\longrightarrow$ 40\% EtOAc in hexanes) to yield 8 (5.15 g, 13.4 mmol, $65 \%$ yield, $1.7: 1 d r$ ) as a yellow foam.

TLC: $\mathrm{R}_{\mathrm{f}}=0.25$ (1:1 EtOAc in hexanes).

bold=major epimer normal=minor epimer $\underline{\text { underline }}=$ superimposed peaks of major and minor epimer

${ }^{1}$ H NMR (600 MHz, MeOD) $\delta \underline{7.72}$ (br s), $\underline{7.42}(\mathrm{dd}, J=8.6,7.4 \mathrm{~Hz}), \underline{7.32-7.25}(\mathrm{~m}), \underline{7.22}(\mathrm{dq}, J$ $=7.9,1.3 \mathrm{~Hz}), \underline{6.95}$ (br s), $\mathbf{5 . 3 3}$ (br s), 5.28 (br s), 4.64 (dd, $J=7.7,6.7 \mathrm{~Hz}), 4.60$ (dd, $J=9.9,3.9$ $\mathrm{Hz}), \underline{4.39-4.23}(\mathrm{~m}), \mathbf{3 . 4 0}(\mathrm{dddd}, J=9.3,4.1,3.2,1.0 \mathrm{~Hz}), 3.34-3.31(\mathrm{~m}), \mathbf{2 . 2 2}-\mathbf{2 . 1 6}(\mathrm{m}), 2.16$ 
$-2.11(\mathrm{~m}), 1.99(\mathrm{dt}, J=13.8,7.8 \mathrm{~Hz}), 1.90(\mathrm{ddd}, J=13.7,9.5,4.0 \mathrm{~Hz}, 2 \mathrm{H}), 1.34(\mathrm{td}, J=7.1,3.9$

$\mathrm{Hz}, 9 \mathrm{H})$.

${ }^{13}$ C NMR (151 MHz, MeOD) $\delta$ 164.8, 164.6, 154.2, 153.9, 151.9, 150.4 (br), 137.1, 136.9,

$\underline{130.6}, \underline{127.5}, \underline{122.8}, \underline{122.5}$ (br), 119.4, 119.2, 111.5 (br), 110.8 (br), $\underline{95.8}$ (br), 68.7, 67.3, 62.5,

62.4, 42.9, $42.8,32.2,31.4,14.5,14.5$.

IR (thin film) $v_{\max }\left(\mathbf{c m}^{-1}\right)$ : 3370 (br), 2925 (w), 2856 (w), 2213 (m), 1738 (s), 1685 (s), 1619 (m), $1344(\mathrm{~m}), 1319(\mathrm{~s}), 1285(\mathrm{~m}), 1184(\mathrm{~s}), 1166(\mathrm{~s}), 1154(\mathrm{~m})$.

HRMS (m/z): (ESI) calcd. for $\mathrm{C}_{19} \mathrm{H}_{19} \mathrm{~N}_{3} \mathrm{O}_{6} \mathrm{Na}[\mathrm{M}+\mathrm{Na}]^{+}:$408.1166, found 408.1165.

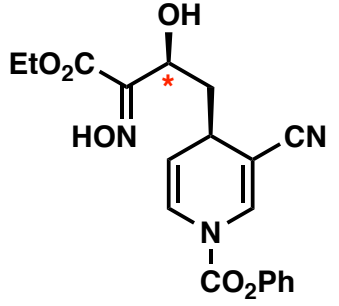

d.r. $1.7: 1$

8

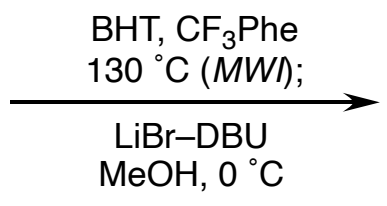

$\mathrm{MeOH}, 0^{\circ} \mathrm{C}$

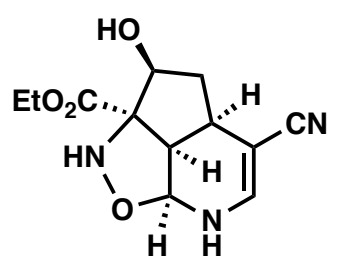

9

Isoxazolidine 9: Six 20-mL Biotage microwave vial were equipped with stir bars and backfilled with nitrogen, to these was added a solution of butylated hydroxytoluene (BHT, 6 x $100 \mathrm{mg}, 2.73$ mmol, 0.35 equiv.) in trifluorotoluene $(6 \times 19 \mathrm{~mL})$, which had been previously degassed by sparging with argon. This was followed by a solution of alcohols 8 and epi-8 $(6$ x $500 \mathrm{mg}, 1.29$ mmol, 1.0 equiv. $)$ in DCM $(6 \times 1 \mathrm{~mL})$. The vials were crimped shut and heated each to $130{ }^{\circ} \mathrm{C}$ for 8 hours in a Biotage microwave reactor over a period of 24 hours. Next a $500-\mathrm{mL}$ flame dried round bottom flask was equipped with a stir bar and oven-dried $3 \AA$ pelleted molecular sieves and put under positive nitrogen pressure. Using a syringe and thick-gauge needle the reaction mixture was transferred to the round bottom flask using methanol $(70 \mathrm{~mL})$ and sonication to completely 
transfer all solids. To this was added lithium bromide (3.38 g, $38.9 \mathrm{mmol}, 5.0$ equiv.) in methanol $(8 \mathrm{~mL})$, and this mixture was allowed to stir for 15 minutes at room temperature. The mixture was then cooled to $0{ }^{\circ} \mathrm{C}$ in an ice bath and DBU was added $(1.16 \mathrm{~mL}, 7.78 \mathrm{mmol}, 1.0$ equiv.) dropwise. Upon reaction completion as indicated by TLC, roughly $2 \mathrm{~h}$, pivalic acid (1.59 g, $15.6 \mathrm{mmol}, 2$ equiv.) in methanol $(5 \mathrm{~mL})$ was transferred to the reaction flask via syringe. The mixture was allowed to warm to room temperature and concentrated in vacuo (rotovap bath no higher than 33 $\left.{ }^{\circ} \mathrm{C}\right)$. The crude mixture was purified by column chromatography $(1 \rightarrow 4 \% \mathrm{MeOH}$ in $\mathrm{DCM}+0.07 \%$ $\mathrm{NH}_{4} \mathrm{OH}$ ) and triturated with ether to remove remaining pivalic acid to yield $\mathbf{9}$ as a fine pale yellow powder (890 mg, $3.50 \mathrm{mmol}$, 45\% yield). The pure material could be crystallized by slow evaporation from hot methanol.

TLC: $\mathrm{R}_{\mathrm{f}}=0.35(10 \% \mathrm{MeOH}$ in 2:1 DCM/hexanes, run twice).

${ }^{1}$ H NMR (500 MHz, MeOD) $\delta 7.00(\mathrm{~s}, 1 \mathrm{H}), 5.00(\mathrm{~d}, J=4.8 \mathrm{~Hz}, 1 \mathrm{H}), 4.46(\mathrm{dd}, J=11.9,6.3 \mathrm{~Hz}$, 1H), $3.82(\mathrm{~s}, 3 \mathrm{H}), 2.79(\mathrm{dd}, J=9.0,4.8 \mathrm{~Hz}, 1 \mathrm{H}), 2.72(\mathrm{td}, J=10.6,9.0,6.3 \mathrm{~Hz}, 1 \mathrm{H}), 2.29(\mathrm{dt}, J=$ 11.9, $6.3 \mathrm{~Hz}, 1 \mathrm{H}), 1.57$ (q, $J=11.9 \mathrm{~Hz}, 1 \mathrm{H})$.

${ }^{13}$ C NMR (151 MHz, MeOD) $\delta 173.4,142.1,122.5,85.6,80.6,78.9,74.7,53.5,47.5,40.6,31.7$. IR (thin film) $v_{\max }\left(\mathbf{c m}^{-1}\right): 3278$ (br), 3214 (m), 3033 (m), 2853 (w), 2193 (s), 1730 (s), 1637 (s), 1384 (s), 1275 (s), 1224 (s), 1053 (s).

HRMS (m/z): (ESI) calcd. for $\mathrm{C}_{11} \mathrm{H}_{14} \mathrm{~N}_{3} \mathrm{O}_{4}[\mathrm{M}+\mathrm{H}]^{+}:$252.0979, found 252.0981 .

The carbamate-protected isoxazolidine (SI-4) can also be isolated:

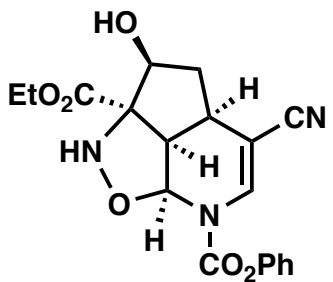

$\mathrm{SI}-4$
TLC: $\mathrm{R}_{\mathrm{f}}=0.57(10 \% \mathrm{MeOH}$ in $2: 1 \mathrm{DCM} /$ Hexanes $)$.

${ }^{1}$ H NMR (700 MHz, MeOD) $\delta 7.80($ br s, $1 \mathrm{H}), 7.43(\mathrm{dd}, J=8.5,7.4 \mathrm{~Hz}$, 2H), $7.31(\mathrm{t}, J=7.5 \mathrm{~Hz}, 1 \mathrm{H}), 7.23(\mathrm{~d}, J=7.6 \mathrm{~Hz}, 2 \mathrm{H}), 5.90$ (br s, 1H), 4.55 $(\mathrm{dd}, J=11.9,6.4 \mathrm{~Hz}, 1 \mathrm{H}), 4.30(\mathrm{qd}, J=7.1,1.3 \mathrm{~Hz}, 2 \mathrm{H}), 3.31(\mathrm{~s}, 1 \mathrm{H}), 3.17$ 
$(\mathrm{dd}, J=9.3,5.0 \mathrm{~Hz}, 1 \mathrm{H}), 2.87(\mathrm{ddd}, J=12.6,9.3,6.4 \mathrm{~Hz}, 1 \mathrm{H}), 2.41(\mathrm{dt}, J=11.9,6.4 \mathrm{~Hz}, 1 \mathrm{H})$, $1.65(\mathrm{q}, J=12.6,11.9 \mathrm{~Hz}, 1 \mathrm{H}), 1.33(\mathrm{t}, J=7.1 \mathrm{~Hz}, 3 \mathrm{H})$.

${ }^{13}$ C NMR (151 MHz, MeOD) $\delta$ 172.3, 152.2 (br), 152.0, 135.3, 130.7, 127.6, 122.5, 119.4, 94.8, 85.0, 78.9, 75.3, 63.6, 49.4-46.8 (under solvent peak, see provided HSQC), 40.2, 31.7, 14.3.

IR (thin film) $v_{\max }\left(\mathbf{c m}^{-1}\right): 3462$ (br), 3238 (br), 2982 (br), 2215 (m), 1735 (s), 1650 (m), 1317 (s), 1197 (s), 1169 (s).

HRMS (m/z): (ESI) calcd. for $\mathrm{C}_{19} \mathrm{H}_{20} \mathrm{~N}_{3} \mathrm{O}_{6} \mathrm{Na}[\mathrm{M}+\mathrm{H}]^{+}:$386.1347, found 386.1344.

Ketone isoxazolidine (SI-5): See general procedure for 9. After microwave reaction the crude mixture was concentrated and purified by preparatory TLC.

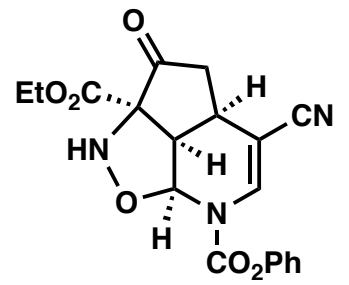

SI-5

TLC: $\mathrm{R}_{\mathrm{f}}=0.3(1: 1 \mathrm{EtOAc} /$ Hexanes $)$.

${ }^{1}$ H NMR (700 MHz, MeOD) $\delta 7.91(\mathrm{~s}, 1 \mathrm{H}), 7.53$ - 7.37 (m, 2H), $7.36-$ $7.28(\mathrm{~m}, 1 \mathrm{H}), 7.28-7.04(\mathrm{~m}, 2 \mathrm{H}), 5.94(\mathrm{~s}, 1 \mathrm{H}), 4.37-4.28(\mathrm{~m}, 2 \mathrm{H}), 3.65$ (dd, $J=9.5,5.7 \mathrm{~Hz}, 1 \mathrm{H}), 2.93(\mathrm{dd}, J=17.6,8.5 \mathrm{~Hz}, 1 \mathrm{H}), 2.43(\mathrm{dd}, J=17.6$,

$11.8 \mathrm{~Hz}, 1 \mathrm{H}), 1.30(\mathrm{t}, J=7.1 \mathrm{~Hz}, 3 \mathrm{H})$.

HRMS (m/z): (ESI) calcd. for $\mathrm{C}_{19} \mathrm{H}_{18} \mathrm{~N}_{3} \mathrm{O}_{6} \mathrm{Na}[\mathrm{M}+\mathrm{H}]^{+}: 384.1190$, found 384.1191.
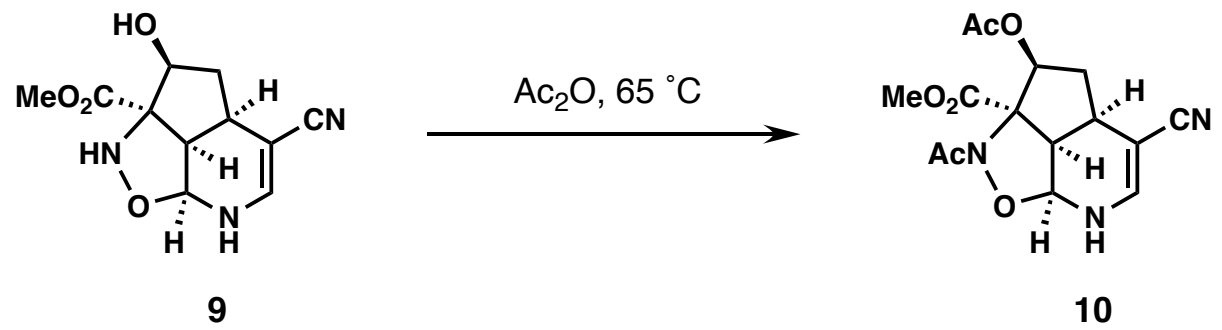

Bisacyl-isoxalidine 10: A 100-mL round bottom flask was loaded with 9 (600 mg, 2.36 mmol, 1.0 equiv.) and acetic anhydride $(12 \mathrm{~mL})$. The resulting mixture was stirred at $65{ }^{\circ} \mathrm{C}$. After 12 
hours, full conversion to product was achieved, as indicated by TLC and NMR of aliquots. The presence of an overlapping less polar spot indicates the mono $O$-acyl reaction intermediate, and steady heating should be maintained to achieve full conversion. The reaction mixture was then cooled to room temperature and concentrated in vacuo. The crude material was purified by column chromatography $(1 \rightarrow 4 \% \mathrm{MeOH}$ in $3: 1 \mathrm{DCM} /$ hexanes $)$ to provide $\mathbf{1 0}$ as a fine pale yellow powder (700 mg, $2.09 \mathrm{mmol}, 89 \%$ yield). On large scales, 10 can be purified by recrystallization from hot methanol.

TLC: $\mathrm{R}^{\mathrm{f}}=0.5(10 \% \mathrm{MeOH}$ in $3: 1 \mathrm{DCM} /$ hexanes $)$.

${ }^{1}$ H NMR (700 MHz, MeOD) $\delta 7.07(\mathrm{~s}, 1 \mathrm{H}), 5.75(\mathrm{dd}, J=12.0,6.6 \mathrm{~Hz}, 1 \mathrm{H}), 5.47(\mathrm{~d}, J=4.7 \mathrm{~Hz}$, 1H), 3.75 (s, 3H), 3.08 (dd, $J=9.9,4.7 \mathrm{~Hz}, 1 \mathrm{H}), 2.85$ (ddd, $J=12.8,9.8,6.7 \mathrm{~Hz}, 1 \mathrm{H}), 2.49$ (dt, $J$ $=12.0,6.6 \mathrm{~Hz}, 1 \mathrm{H}), 2.13(\mathrm{~s}, 3 \mathrm{H}), 1.98(\mathrm{~s}, 3 \mathrm{H}), 1.63(\mathrm{q}, J=12.8,12.0 \mathrm{~Hz}, 1 \mathrm{H})$.

${ }^{13}$ C NMR (151 MHz, MeOD) $\delta 171.8,169.7,169.3,141.8,121.5,84.1,81.6,76.8,73.1,53.5$, $49.0,48.5,37.1,30.5,20.7,20.7$.

IR (thin film) $v_{\max }\left(\mathbf{c m}^{-1}\right): 3376($ br), 3041(w), $2956(w), 2925$ (w), $2853(w), 2201(\mathrm{~m}), 1745$ (s), 1647 9s), $1435(\mathrm{~m}), 1265(\mathrm{~s}), 1237(\mathrm{~s})$.

HRMS (m/z): (ESI) calcd. for $\mathrm{C}_{15} \mathrm{H}_{17} \mathrm{~N}_{3} \mathrm{O}_{6} \mathrm{Na}[\mathrm{M}+\mathrm{Na}]^{+}:$358.1010, found 358.1008.

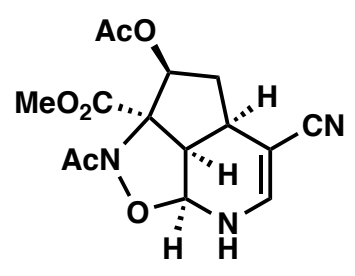

10

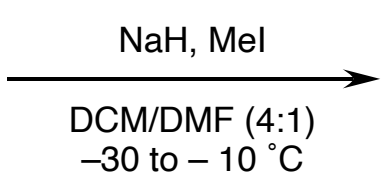

-30 to $-10^{\circ} \mathrm{C}$

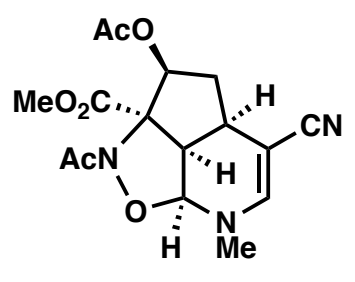

11

$N$-methyl isoxazolidine 11: A suspension of $\mathrm{NaH}(90 \%, 615 \mathrm{mg}, 25.6 \mathrm{mmol}, 20$ equiv.) in DCM $(30 \mathrm{~mL})$ was cooled to $-30{ }^{\circ} \mathrm{C}$ and to this was added a suspension of $\mathbf{1 0}$ (430 $\mathrm{mg}, 1.28 \mathrm{mmol}, 1.0$ 
equiv.) and oven-dried powdered $3 \AA$ molecular sieves ( $\sim 50 \mathrm{mg})$ in DCM (20 mL) and DMF (12 $\mathrm{mL}$ ) under positive nitrogen pressure. This was rapidly followed by addition of iodomethane (3.2 $\mathrm{mL}, 51.3 \mathrm{mmol}, 40$ equiv.), which had itself been stirred for several minutes over $\mathrm{P}_{2} \mathrm{O}_{5}$. The reaction is extremely moisture-sensitive. The reaction mixture was allowed to warm to $-10{ }^{\circ} \mathrm{C}$ and stirred at this temperature until reaction completion as indicated by TLC, approximately 2 hours. The mixture was carefully poured into ice cold $1 \mathrm{M} \mathrm{HCl}(30 \mathrm{~mL})$, after a few minutes, the mixture was neutralized to $\mathrm{pH} 7$ by slow addition of solid $\mathrm{NaHCO}_{3}(2.5 \mathrm{~g})$. The biphasic mixture was allowed to warm to room temperature and extracted with DCM $(3 \times 10 \mathrm{~mL})$, washed with brine, dried over $\mathrm{Na}_{2} \mathrm{SO}_{4}$, and concentrated in vacuo. The resulting yellow oil was purified by column chromatography $(1 \rightarrow 4 \% \mathrm{MeOH}$ in $2: 1 \mathrm{DCM} / \mathrm{Hexanes})$ to yield the desired product as a pale yellow solid (440 mg, $1.26 \mathrm{mmol}, 98 \%$ yield). 11 can be crystallized by slow evaporation from methanol.

TLC: $\mathrm{R}_{\mathrm{f}}=0.7(10 \%$ Methanol in 2:1 DCM/Hexanes).

${ }^{1}$ H NMR (600 MHz, MeOD) $\delta 7.05$ (s, 1H), 5.75 (dd, $\left.J=11.7,6.4 \mathrm{~Hz}, 1 \mathrm{H}\right), 5.39$ (d, $J=4.8 \mathrm{~Hz}$, 1H), $3.75(\mathrm{~s}, 3 \mathrm{H}), 3.19(\mathrm{~s}, 3 \mathrm{H}), 3.16(\mathrm{dd}, J=9.5,4.4 \mathrm{~Hz}, 1 \mathrm{H}), 2.80(\mathrm{ddd}, J=12.8,10.0,6.8 \mathrm{~Hz}$, 1H), $2.48(\mathrm{dt}, J=11.6,6.5 \mathrm{~Hz}, 1 \mathrm{H}), 2.15(\mathrm{~s}, 3 \mathrm{H}), 1.97(\mathrm{~s}, 3 \mathrm{H}), 1.58(\mathrm{dt}, J=12.9,11.7 \mathrm{~Hz}, 1 \mathrm{H})$. ${ }^{13}$ C NMR (151 MHz, MeOD) $\delta 171.7,169.8,169.2,145.6,121.4,89.6,81.6,76.7,73.3,53.5$, 49.1 (behind solvent peak, see HSQC data), 41.9, 37.3, 29.7, 20.7, 20.7.

HRMS (m/z): (ESI) calcd. for $\mathrm{C}_{16} \mathrm{H}_{19} \mathrm{~N}_{3} \mathrm{O}_{6} \mathrm{Na}[\mathrm{M}+\mathrm{Na}]^{+}: 372.1166$, found 372.1170.

IR (thin film) $\mathbf{v}_{\max }\left(\mathbf{c m}^{-1}\right): 2956(\mathrm{w}), 2925$ (w), 2855 (w), 2200 (m), 1749 (s), 1671 (m), 1645 (s), $1436(\mathrm{~m}), 1237(\mathrm{~s})$ 


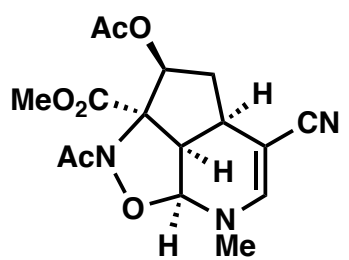

11

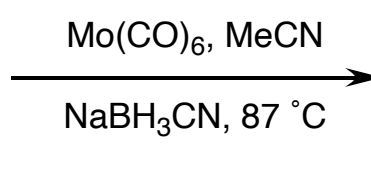

$\mathrm{R}=\mathrm{Ac}$ and $\mathrm{H}$

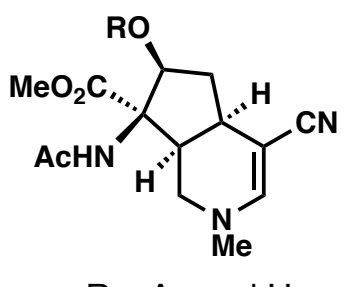

12

Acylamine 12: [CAUTION: $\mathrm{Mo}(\mathrm{CO})_{6}, \mathrm{NaBH}_{3} \mathrm{CN}$, AND THEIR BYPRODUCTS SHOULD BE HANDLED WITH CARE IN A WELL-VENTILATED FUME HOOD]. A 25-mL three-necked round bottom flask was sealed with two rubber septa, equipped with a reflux condenser, and loaded with hexacarbonylmolybdenum (160 mg, $0.59 \mathrm{mmol}, 1.01$ equiv.). To this was added $\mathrm{MeCN}(3 \mathrm{~mL})$ and the resulting solution was refluxed in a $95{ }^{\circ} \mathrm{C}$ oil bath for $4-6$ hours during which time the mixture turned from pale to dark brown. Separately, a 50-mL round bottom flask was loaded with 11 (203 mg, $0.58 \mathrm{mmol}, 1.0$ equiv.) and sodium cyanoborohydride (527 mg, 8.40 mmol, 15.0 equiv.), which were dissolved in $\mathrm{MeCN}$ (3 mL) and transferred dropwise to the refluxing reaction mixture. The resulting dark brown reaction mixture was allowed to reflux until consumption of starting material 11, as indicated by TLC. The mixture was cooled to room temperature and carefully poured into a $0{ }^{\circ} \mathrm{C} a q$. phosphate buffer solution $(1 \mathrm{M}, \mathrm{pH} 7)$ with vigorous stirring. The biphasic mixture was allowed to warm to room temperature with continued stirring and transferred to a separatory funnel. The aqueous layer was extracted with ${ }^{i} \mathrm{PrOH} /$ chloroform $(1: 3,3 \times 300 \mathrm{~mL})$. The combined organic layers were washed with brine $(1 \mathrm{x}$ $300 \mathrm{~mL}$ ), dried over Na2SO4, and concentrated in vacuo. The resulting pale brown oil was purified by column chromatography $(3 \% \rightarrow 6 \% \mathrm{MeOH}$ in $2: 1 \mathrm{DCM} /$ hexanes $)$ to yield an inconsequential mixture of bis-acyl and mono-acyl product 12 (160 mg, $0.48 \mathrm{mmol}, 82 \%$ yield) as a pale brown powder.

$\underline{\mathrm{R}=\mathrm{Ac}}$ 
TLC: $\mathrm{R}_{\mathrm{f}}=0.6(10 \%$ Methanol in 2:1 DCM/Hexanes).

${ }^{1}$ H NMR (600 MHz, MeOD) $\delta 6.95(\mathrm{~d}, J=1.0 \mathrm{~Hz}, 1 \mathrm{H}), 5.41(\mathrm{t}, J=7.7 \mathrm{~Hz}, 1 \mathrm{H}), 3.67(\mathrm{~s}, 3 \mathrm{H}), 3.23$ $(\mathrm{dd}, J=13.0,6.9 \mathrm{~Hz}, 1 \mathrm{H}), 3.07(\mathrm{ddd}, J=13.0,5.4,1.0 \mathrm{~Hz}, 1 \mathrm{H}), 2.94(\mathrm{td}, J=7.2,5.5 \mathrm{~Hz}, 1 \mathrm{H})$, $2.80(\mathrm{q}, J=7.8 \mathrm{~Hz}, 1 \mathrm{H}), 2.63(\mathrm{dt}, J=13.9,8.3 \mathrm{~Hz}, 1 \mathrm{H}), 2.07(\mathrm{~s}, 3 \mathrm{H}), 1.98(\mathrm{~s}, 3 \mathrm{H}), 1.76(\mathrm{dt}, J=$ $13.8,7.6 \mathrm{~Hz}, 1 \mathrm{H})$.

${ }^{13}$ C NMR (151 MHz, MeOD) $\delta$ 173.8, 173.3, 171.2, 149.2, 123.9, 77.6, 75.8, 68.4, 53.1, 45.7, $42.6,41.9,38.2,31.8,22.0,20.8$.

HRMS (m/z): (ESI) calcd. for $\mathrm{C}_{16} \mathrm{H}_{22} \mathrm{~N}_{3} \mathrm{O}_{5}[\mathrm{M}+\mathrm{H}]^{+}: 336.1565$, found 336.1560 .

IR (thin film) $v_{\max }\left(\mathbf{c m}^{-1}\right)$ : 3332 (br), 2925 (w), 2184 (m), 1737 (s), 1663 (m), 1629 (s), 1520 (w), $1425(w), 1372(w), 1237(s)$.

$\underline{\mathrm{R}=\mathrm{H}}$

TLC: $\mathrm{R}_{\mathrm{f}}=0.5$ (10\% Methanol in 2:1 DCM/Hexanes).

${ }^{1}$ H NMR (600 MHz, MeOD) $\delta 6.97(\mathrm{~s}, 1 \mathrm{H}), 4.17(\mathrm{t}, J=7.9 \mathrm{~Hz}, 1 \mathrm{H}), 3.69(\mathrm{~s}, 3 \mathrm{H}), 2.96$ - 2.95 (m, 1H), $2.91(\mathrm{~s}, 3 \mathrm{H}), 2.82-2.76(\mathrm{~m}, 1 \mathrm{H}), 2.69(\mathrm{dt}, J=11.2,6.6 \mathrm{~Hz}, 1 \mathrm{H}), 2.53(\mathrm{dt}, J=13.1,7.3 \mathrm{~Hz}$, 1H), 2.03 (s, 3H), 1.45 (ddd, $J=13.1,11.1,8.4 \mathrm{~Hz}, 1 \mathrm{H})$.

${ }^{13}$ C NMR (151 MHz, MeOD) $\delta$ 175.0, 173.7, 149.5, 124.3, 76.2, 75.7, 68.1, 53.0, 45.6, 42.8, 42.4, $41.1,33.4,22.2$.

HRMS (m/z): (ESI) calcd. for $\mathrm{C}_{14} \mathrm{H}_{19} \mathrm{~N}_{3} \mathrm{O}_{4} \mathrm{Na}[\mathrm{M}+\mathrm{Na}]^{+}: 316.1268$, found 316.1269 .

IR (thin film) $v_{\max }\left(\mathbf{c m}^{-1}\right)$ : 3349 (br), 2200 (m), 1734 (m), 1640 (s), 1498 (w), 1434 (w), 1412 (w), $1351(\mathrm{w}), 1295(\mathrm{~m})$. 


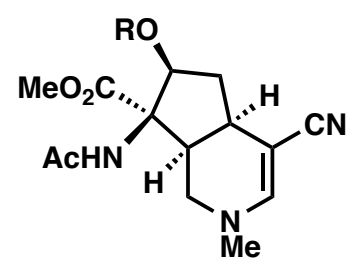

$\mathrm{R}=\mathrm{Ac}$ and $\mathrm{H}$

12

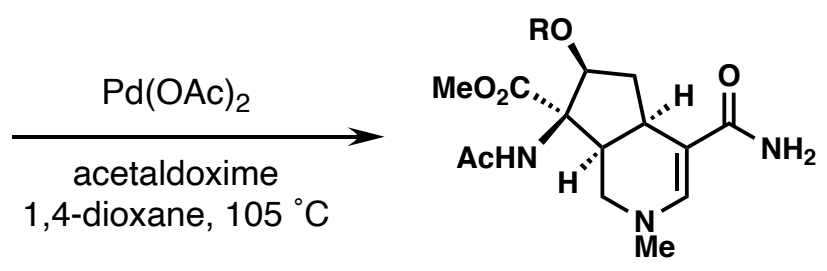

$\mathrm{R}=\mathrm{Ac}$ and $\mathrm{H}$

13

Amide 13: A 50-mL two-necked round bottom flask bottom flask equipped with a reflux condenser was loaded with nitrile 12 (1.9:1 molar ratio, $471 \mathrm{mg}, 1.78 \mathrm{mmol}, 1.0$ equiv.), palladium(II) acetate (40 mg, $0.178 \mathrm{mmol}, 10 \mathrm{~mol} \%$ ), acetaldoxime (300 mg, $17.8 \mathrm{mmol}, 10$. equiv.), and 1,4-dioxane $(24 \mathrm{~mL})$. The mixture was degassed with argon and refluxed in a $105{ }^{\circ} \mathrm{C}$ oil bath. Upon reaction completion, roughly 12 hours, as indicated by the consumption of starting material by TLC visualization, the reaction mixture was cooled to room temperature, filtered through Celite ${ }^{\circledR}$, and concentrated in vacuo. The crude product was purified by column chromatography $\left(4 \% \rightarrow 8 \% \mathrm{MeOH}\right.$ with $\mathrm{NH}_{4} \mathrm{OH}$ in DCM) to yield $\mathbf{1 3} O$-acyl amide (364 mg, 1.03 mmol). and $O$-deacyl amide $(132 \mathrm{mg}, 0.424 \mathrm{mmol})$ with an overall yield of $82 \%$ yield. NOTE: care must be taken to completely remove the acetamide byproduct which is formed from rearrangement of acetaldoxime and is noticeable in ${ }^{1} \mathrm{H}$ NMRs at $1.8 \mathrm{ppm}$.

$\underline{\mathrm{R}=\mathrm{Ac}}$

TLC: $\mathrm{R}_{\mathrm{f}}=0.3(10 \%$ Methanol in 3:1 DCM/Hexanes).

${ }^{1}$ H NMR (600 MHz, MeOD) $\delta 7.31(\mathrm{~s}, 1 \mathrm{H}), 5.46(\mathrm{t}, J=7.2 \mathrm{~Hz}, 1 \mathrm{H}), 3.70(\mathrm{~s}, 3 \mathrm{H}), 3.09$ - $2.98(\mathrm{~m}$, 2H), 2.97 (s, 3H), $2.93(\mathrm{dd}, J=10.2,7.2 \mathrm{~Hz}, 1 \mathrm{H}), 2.89-2.79(\mathrm{~m}, 2 \mathrm{H}), 2.06(\mathrm{~s}, 3 \mathrm{H}), 2.01(\mathrm{~s}, 3 \mathrm{H})$, $1.40(\mathrm{ddd}, J=13.7,10.3,6.8 \mathrm{~Hz}, 1 \mathrm{H})$.

${ }^{13}$ C NMR (151 MHz, MeOD) $\delta$ 174.0, 173.9, 173.7, 171.1, 145.9, 100.8, 75.9, 68.8, 53.2, 45.6, $43.0,42.4,40.7,31.8,21.9,20.9$. 
IR (thin film) $v_{\max }\left(\mathbf{c m}^{-1}\right): 3352$ (br), 2951 (w), 1730 (s), 1645 (s), 1650 (s), 1538 (m), 1291 (m), $1245(\mathrm{~s})$.

HRMS (m/z): (ESI) calcd. for $\mathrm{C}_{16} \mathrm{H}_{23} \mathrm{~N}_{3} \mathrm{O}_{6} \mathrm{Na}[\mathrm{M}+\mathrm{Na}]^{+}:$376.1490, found 376.1487.

$\underline{\mathrm{R}=\mathrm{H}}$

TLC: $\mathrm{R}_{\mathrm{f}}=0.2(10 \%$ Methanol in 3:1 DCM/Hexanes).

${ }^{1}$ H NMR (600 MHz, MeOD) $\delta 7.33(\mathrm{~s}, 1 \mathrm{H}), 4.19(\mathrm{dd}, J=8.7,7.2 \mathrm{~Hz}, 1 \mathrm{H}), 3.70(\mathrm{~s}, 3 \mathrm{H}), 2.96$ (s, $3 \mathrm{H}), 2.96-2.85(\mathrm{~m}, 2 \mathrm{H}), 2.87(\mathrm{q}, J=12.5,11.8 \mathrm{~Hz}, 2 \mathrm{H}), 2.72(\mathrm{dd}, J=12.0,5.2 \mathrm{~Hz}, 1 \mathrm{H}), 2.63(\mathrm{dt}$, $J=13.0,6.8 \mathrm{~Hz}, 1 \mathrm{H}), 2.05(\mathrm{~s}, 3 \mathrm{H}), 1.24(\mathrm{td}, J=12.6,8.7 \mathrm{~Hz}, 1 \mathrm{H})$.

${ }^{13}$ C NMR (151 MHz, MeOD) $\delta 175.4,173.9,173.6,146.1,100.0,75.8,67.7,53.0,45.8,43.1$, $43.1,41.4,32.3,22.2$.

IR (thin film) $v_{\max }\left(\mathbf{c m}^{-1}\right): 3348$ (br), 3284 (br), 2955 (w), 2923 (w), 1834 (m), 1650 (s), 1530 (s), $1434(\mathrm{~m}), 1381(\mathrm{~m}), 1289(\mathrm{~s})$.

HRMS (m/z): (ESI) calcd. for $\mathrm{C}_{14} \mathrm{H}_{21} \mathrm{~N}_{3} \mathrm{O}_{5} \mathrm{Na} \quad[\mathrm{M}+\mathrm{Na}]^{+}$: 334.1373, found 334.1377.

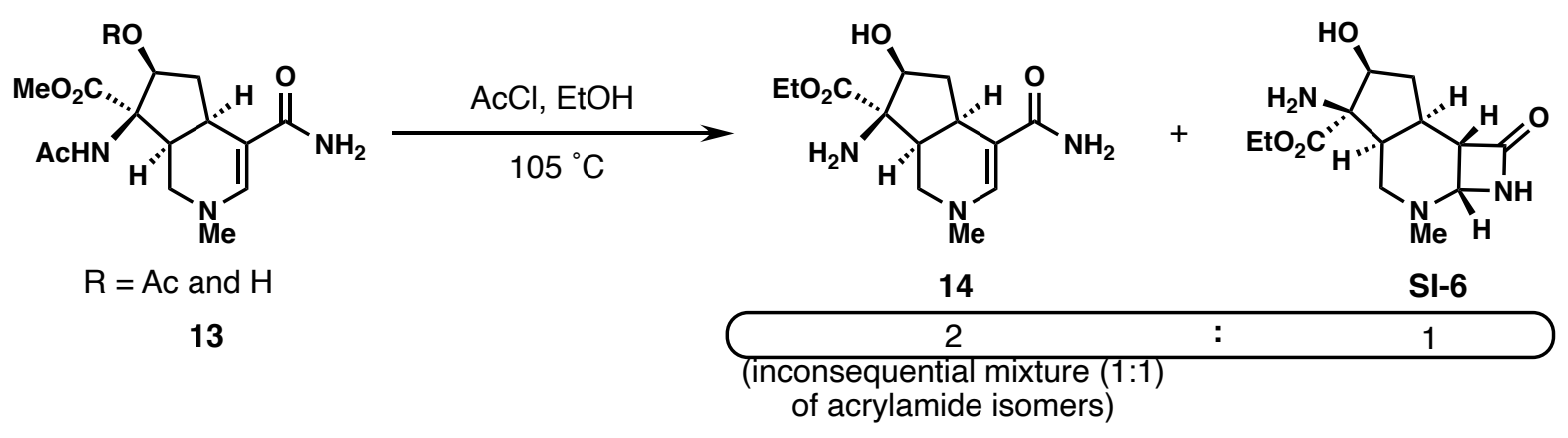

Amino alcohol 14: Amide 13 (26 mg, $0.084 \mathrm{mmol}, 1.0$ equiv.) was concentrated with $3 \times 1 \mathrm{~mL}$ toluene, dried thoroughly on hi-vac, and transferred as a solution in EtOH ( $8.4 \mathrm{~mL})$ to a large flame dried microwave vial equipped with a stir bar under positive nitrogen pressure. To this solution was added freshly distilled acetyl chloride $(0.90 \mathrm{~mL}, 1.5 \mathrm{M})$ dropwise at room temperature. The 
nitrogen line was removed and the microwave vial was carefully sealed with a new, unperforated cap. The reaction was submerged in a $105{ }^{\circ} \mathrm{C}$ oil bath and heated for $1.75 \mathrm{~h}$. Upon reaction completion as determined by TLC and removal of an aliquot and NMR analysis, the pale brown solution was cooled and concentrated in vacuo to yield a brown oil. The crude product was purified by column chromatography $\left(5,10,15,207 \mathrm{~N} \mathrm{NH}_{3} / \mathrm{MeOH}\right.$ in $\left.\mathrm{DCM}\right)$ to yield $14(15 \mathrm{mg}, 0.053$ mmol, $63 \%$ yield) as an inconsequential mixture of two isomers converging over time in $\mathrm{MeOH}$ or by being subjected to the subsequent reaction conditions. The isomers are chromatographically separable and can be collected either separately or together.

TLC: $\mathrm{R}_{\mathrm{f}}=0.46$ ( isomer A), 0.18 (isomer B) $\left(15 \% 7 \mathrm{~N} \mathrm{NH}_{3} / \mathrm{MeOH}\right.$ in DCM)

\section{$\underline{14-I s o m e r ~ A ~}$}

${ }^{1}$ H NMR (500 MHz, MeOD) $\delta 7.28(\mathrm{~s}, 1 \mathrm{H}), 4.29(\mathrm{dd}, J=8.7,7.2 \mathrm{~Hz}, 1 \mathrm{H}), 4.22(\mathrm{q}, J=7.1 \mathrm{~Hz}$, 2H), $3.04(\mathrm{dd}, J=12.7,5.7 \mathrm{~Hz}, 1 \mathrm{H}), 3.00-2.92(\mathrm{~m}, 1 \mathrm{H}), 2.96(\mathrm{~s}, 3 \mathrm{H}), 2.86(\mathrm{dt}, J=10.7,7.4 \mathrm{~Hz}$, 1H), $2.65(\mathrm{dt}, J=13.0,7.2 \mathrm{~Hz}, 1 \mathrm{H}), 2.56(\mathrm{td}, J=8.3,5.7 \mathrm{~Hz}, 1 \mathrm{H}), 1.36-1.32(\mathrm{~m}, 1 \mathrm{H}), 1.30(\mathrm{t}, J$ $=7.1 \mathrm{~Hz}, 3 \mathrm{H})$.

${ }^{13}$ C NMR (151 MHz, MeOD) $\delta 177.0,174.0,146.0,102.0,76.6,68.4,62.6,46.0,43.4,43.0,42.2$, $31.2,14.4$

HRMS (m/z): (ESI) calcd. for $\mathrm{C}_{13} \mathrm{H}_{23} \mathrm{~N}_{3} \mathrm{O}_{4}[\mathrm{M}+\mathrm{H}]^{+}: 284.1605$, found 284.1606.

IR (thin film) $v_{\max }\left(\mathbf{c m}^{-1}\right): 3352$ (br), 3237 (br), 2940 (w), 1722 (m), 1643 (s), 1551 (m), 1386 (w), $1340(\mathrm{w}), 1290(\mathrm{w})$.

14-Isomer B

(See NMR appendix B for evidence of slow conversion) 
${ }^{1}$ H NMR (600 MHz, MeOD) $\delta 7.29(\mathrm{~s}, 1 \mathrm{H}), 4.57(\mathrm{dd}, J=10.4,3.6 \mathrm{~Hz}, 1 \mathrm{H}), 4.38-4.24$ (m, 2H), $3.02-2.92(\mathrm{~m}, 3 \mathrm{H}), 2.74(\mathrm{dt}, J=9.3,3.7 \mathrm{~Hz}, 1 \mathrm{H}), 2.70(\mathrm{~s}, 3 \mathrm{H}), 2.52(\mathrm{ddd}, J=13.6,10.5,6.2 \mathrm{~Hz}$, 1H), $1.80(\mathrm{dd}, J=13.6,3.5 \mathrm{~Hz}, 1 \mathrm{H}), 1.35(\mathrm{t}, J=7.1 \mathrm{~Hz}, 3 \mathrm{H})$.

${ }^{13}$ C NMR (151 MHz, MeOD) 173.4, 171.8, 141.1, 104.3, 83.6, 70.2, 63.5, 47.6, 46.1, 38.4, 36.8, 34.4, 14.4 .

B-lactam SI-6:

TLC: $\mathrm{R}_{\mathrm{f}}=0.9\left(15 \% 7 \mathrm{~N} \mathrm{NH}_{3} / \mathrm{MeOH}\right.$ in $\left.\mathrm{DCM}\right)$

${ }^{1}$ H NMR (500 MHz, MeOD) $\delta 4.31(\mathrm{dd}, J=9.5,3.3 \mathrm{~Hz}, 1 \mathrm{H}), 4.26(\mathrm{qd}, J=7.1,1.2 \mathrm{~Hz}, 2 \mathrm{H}), 3.57$ $(\mathrm{d}, J=1.8 \mathrm{~Hz}, 1 \mathrm{H}), 2.97(\mathrm{dd}, J=12.4,1.8 \mathrm{~Hz}, 1 \mathrm{H}), 2.55(\mathrm{dd}, J=12.1,3.1 \mathrm{~Hz}, 1 \mathrm{H}), 2.42(\mathrm{ddd}, J=$ 14.0, 9.9, 6.9 Hz, 1H), $2.35(\mathrm{~s}, 3 \mathrm{H}), 2.24(\mathrm{t}, J=5.4 \mathrm{~Hz}, 1 \mathrm{H}), 2.21(\mathrm{dd}, J=4.9,2.5 \mathrm{~Hz}, 1 \mathrm{H}), 1.29$ $(\mathrm{t}, J=7.1 \mathrm{~Hz}, 3 \mathrm{H}), 1.29-1.26(\mathrm{~m}, 1 \mathrm{H}), 1.26(\mathrm{dd}, J=13.8,3.5 \mathrm{~Hz}, 1 \mathrm{H})$.

${ }^{13}$ C NMR (151 MHz, MeOD) $\delta$ 175.7, 175.2, 77.4, 68.2, 65.6, 62.9, 49.*, 44.2, 42.0, 40.9, 35.0, 30.4, 14.5. *behind solvent peak, identified by HSQC

Stereochemical determination correlations are shown in NOE spectrum in Appendix B.

HRMS (m/z): (ESI) calcd. for $\mathrm{C}_{13} \mathrm{H}_{22} \mathrm{~N}_{3} \mathrm{O}_{4}[\mathrm{M}+\mathrm{H}]^{+}: 284.1605$, found 284.1607, also found $\mathrm{C}_{12} \mathrm{H}_{21} \mathrm{~N}_{2} \mathrm{O}_{3}[\mathrm{M}-(\mathrm{N}=\mathrm{C}=\mathrm{O})]^{+}:$241.1547, found 241.1545.

IR (thin film) $\mathbf{v}_{\max }\left(\mathbf{c m}^{-1}\right): 3331$ (br), 2936 (m), 2857 (m), 1724 (s), 1653 (m), 1445 (m), 1271 (s), 1215(s), $1084(\mathrm{~s})$.

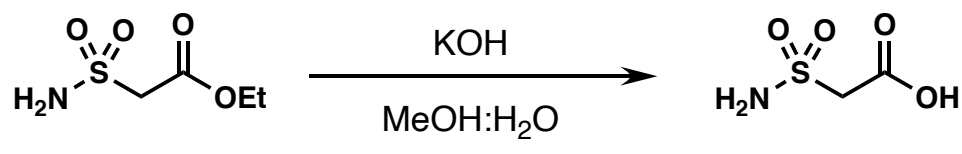

15

2-sulfamoylacetic acid 15: (Ethoxycarbonyl)methanesulfonamide ${ }^{1}$ (60 mg, $0.359 \mathrm{mmol}, 1.0$ equiv.) was dissolved in $\mathrm{MeOH} / \mathrm{H}_{2} \mathrm{O}(1: 1,3.6 \mathrm{~mL})$ and to this was added crushed potassium 
hydroxide (60 mg, $1.08 \mathrm{mmol}, 3.0$ equiv.). After $120 \mathrm{~min}$ the reaction was complete, as indicated by TLC. The reaction was carefully acidified to $\mathrm{pH} 2$ with aq. $1 \mathrm{M} \mathrm{HCl}(1.5 \mathrm{~mL})$ and the mixture was concentrated to dryness. The resulting white powder was resuspended in ${ }^{i} \mathrm{PrOH} / \mathrm{CHCl}_{3}(1: 3)$ $(10 \mathrm{~mL})$ with the aid of sonication, filtered, concentrated in vacuo to yield 2-sulfamoyl acetic acid 15 (50 mg, $0.357 \mathrm{mmol}, 99 \%$ yield) as a white powder.

TLC: $\mathrm{R}_{\mathrm{f}}=0.05(10 \% \mathrm{MeOH} / \mathrm{DCM})$.

${ }^{1}$ H NMR (600 MHz, MeOD) $\delta 4.05$ (s, 2H).

${ }^{13}$ C NMR (151 MHz, MeOD) $\delta 167.6,59.8$.

HRMS (m/z): (ESI) calcd. for $\mathrm{C}_{2} \mathrm{H}_{4} \mathrm{NO}_{4} \mathrm{~S}[\mathrm{M}-\mathrm{H}]^{-:}$: 137.9867, found 137.9825 .

IR (thin film) $v_{\max }\left(\mathbf{c m}^{-1}\right): 3534$ (br), 3363 (br), 3269 (br), 2930 (w), 1725 (s), 1333 (s), 1160 (s), $1138(\mathrm{~s})$

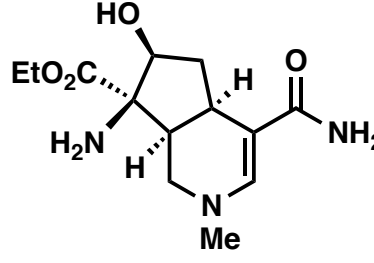

14

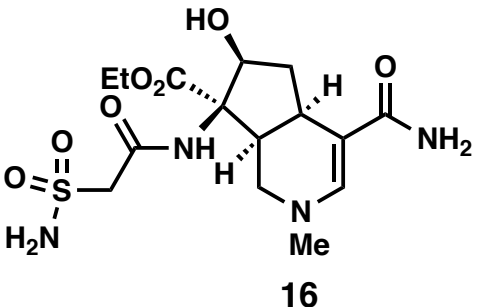

16

Ethyl altemicidin 16: Isomers A and B of amino alcohol 14 (46 mg, $0.162 \mathrm{mmol}, 1.0$ equiv.) was dissolved in DMF (1.5 mL). To this solution was added sidechain 15 (22 mg, $0.17 \mathrm{mmol}, 1.1$ equiv.), DMAP (21 mg, 0.017 mmol, 1.1 equiv.), and N,N'-dicyclohexylcarbodiimide (DCC, 33 $\mathrm{mg}, 0.17 \mathrm{mmol}, 1.1$ equiv.). The reaction was stirred for at least 12 hours or until TLC indicated consumption of starting material and concentrated to dryness in vacuo. The yellow residue was resuspended in distilled water with the aid of sonication and filtered through a pad of Celite ${ }^{\circledR}$ to remove the dicyclohexylurea byproduct. The resulting aqueous solution was concentrated in vacuo 
and purified column chromatography $\left(5,10,157 \mathrm{~N} \mathrm{NH}_{3} / \mathrm{MeOH}\right.$ in $\left.\mathrm{DCM}\right)$. The desired 16 was isolated as a pale yellow oil (48 $\mathrm{mg}, 0.12 \mathrm{mmol}, 73 \%$ yield).

TLC: $\mathrm{R}_{\mathrm{f}}=0.22\left(20 \%\right.$ Methanol, $2 \% \mathrm{NH}_{4} \mathrm{OH}$ in DCM $)$ or $\mathrm{R}_{\mathrm{f}}=0.18\left(15 \% 7 \mathrm{~N} \mathrm{NH}_{3} / \mathrm{MeOH}\right.$ in DCM $)$ ${ }^{1}$ H NMR (600 MHz, MeOD) $\delta 7.34(\mathrm{~s}, 1 \mathrm{H}), 4.22(\mathrm{dd}, J=8.4,7.2 \mathrm{~Hz}, 1 \mathrm{H}), 4.21(\mathrm{~d}, J=14.2 \mathrm{~Hz}$, 1H, exchangeable), 4.18 (q, $J=7.1 \mathrm{~Hz}, 2 \mathrm{H}), 4.10(\mathrm{~d}, J=14.2 \mathrm{~Hz}, 1 \mathrm{H}$, exchangeable), $2.96(\mathrm{~s}, 3 \mathrm{H})$, $2.95-2.81(\mathrm{~m}, 4 \mathrm{H}), 2.66(\mathrm{dt}, J=12.9,6.9 \mathrm{~Hz}, 1 \mathrm{H}), 1.30-1.25(\mathrm{~m}, 1 \mathrm{H}), 1.26(\mathrm{t}, J=7.1 \mathrm{~Hz}, 3 \mathrm{H})$. ${ }^{13}$ C NMR (151 MHz, MeOD) $\delta$ 174.5, 174.0, 165.2, 146.1, 99.7, 75.9, 68.0, 62.8, 60.7, 45.8, 43.2, $43.0,41.4,32.6,14.4$.

HRMS (m/z): (ESI) calcd. for $\mathrm{C}_{15} \mathrm{H}_{25} \mathrm{~N}_{4} \mathrm{O}_{7} \mathrm{~S}[\mathrm{M}+\mathrm{H}]+$ : 405.1438 , found 405.1439 .

Deuterated compound: $\mathrm{C}_{15} \mathrm{H}_{23} \mathrm{D}_{2} \mathrm{~N}_{4} \mathrm{O}_{7} \mathrm{~S}[\mathrm{M}+\mathrm{H}]+:$ 407.1564, found 407.1565.

IR (thin film) $v_{\max }\left(\mathbf{c m}^{-1}\right): 3133$ (br), 3041 (br), 2926 (m), 2853 (m), 1646 (s), 1403 (s), 1338 (m), $1203(\mathrm{~m}), 1182(\mathrm{w}), 1160(\mathrm{~m}), 1134(\mathrm{~m})$.

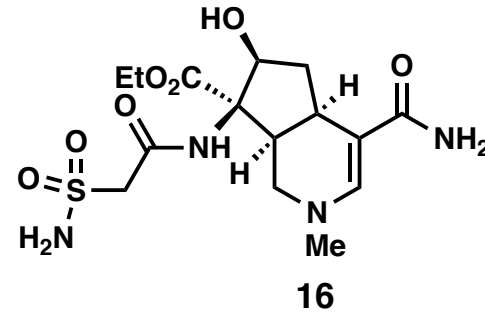

Altemicidin 1: Ethyl altemicidin $16(11 \mathrm{mg}, 0.027 \mathrm{mmol}, 1.0$ equiv.) was transferred to a reaction tube with methanol and concentrated three times with toluene to remove any residual water. The reaction tube was equipped with a stir bar, brought into a glove box, and loaded with LiI (45 mg, $0.34 \mathrm{mmol}, 12$. equiv.). Pyridine $(1 \mathrm{~mL})$ was added to the reaction tube, which was sealed with parafilm and wrapped tightly in foil before being placed in a $130{ }^{\circ} \mathrm{C}$ oil bath for 9 hours. After 9 hours the reaction mixture was cooled to room temperature and concentrated down at room 
temperature by blowing down with nitrogen. The resulting brown solid was resuspended in 1:1 DCM/Ethyl acetate and filtered through Celite ${ }^{\circledR}$. The filtrate was discarded and the remaining solid was eluted into a fresh flask with 1:1 methanol/water and concentrated by blowing down with nitrogen. The crude mixture was loaded onto a C18 reverse phase column and eluted with 5\% $\mathrm{MeOH}$ in $10 \mathrm{mM} \mathrm{NH}_{4} \mathrm{OAc}$ (aq.). Fractions containing altemicidin-oxazoline were heated for 10$12 \mathrm{~h}$ at $60{ }^{\circ} \mathrm{C}$ to produce altemicidin. Combined fractions were subjected to a second $\mathrm{C} 18$ reverse phase column to remove the $\mathrm{NH}_{4} \mathrm{OAc}\left(5 \% \mathrm{MeCN} / \mathrm{H}_{2} \mathrm{O}\right)$ to yield altemicidin $\mathbf{1}$ ( $7 \mathrm{mg}, 0.018 \mathrm{mmol}$, $67 \%$ yield) as a white powder.

TLC: $\mathrm{R}_{\mathrm{f}}=0.2\left(25 \% 7 \mathrm{~N} \mathrm{NH}_{3} /\right.$ Methanol in DCM $)$

${ }^{1}$ H NMR $\left(700 \mathrm{MHz}, \mathrm{D}_{2} \mathrm{O}\right) \delta 7.39(\mathrm{~s}, 1 \mathrm{H}), 4.38(\mathrm{~d}, J=14.1 \mathrm{~Hz}, 1 \mathrm{H}), 4.29(\mathrm{~d}, J=14.1 \mathrm{~Hz}, 1 \mathrm{H})$, $4.28(\mathrm{~m}, 1 \mathrm{H}), 2.98(\mathrm{~s}, 2 \mathrm{H}), 2.92(\mathrm{dd}, J=13.3,7.2 \mathrm{~Hz}, 1 \mathrm{H}), 2.89-2.81(\mathrm{~m}, 3 \mathrm{H}), 2.67(\mathrm{~m}, 1 \mathrm{H}), 1.26$ $(\mathrm{dt}, J=12.8,8.8 \mathrm{~Hz}, 1 \mathrm{H})$.

${ }^{13}$ C NMR $\left(226 \mathrm{MHz}, \mathrm{D}_{2} \mathrm{O}\right) \delta 179.7,174.2,164.4,147.3,97.1,76.0,69.1,60.1,45.5,43.2,41.3$, $40.8,31.7$.

HRMS (m/z): (ESI) calcd. for $\mathrm{C}_{13} \mathrm{H}_{19} \mathrm{~N}_{4} \mathrm{O}_{7} \mathrm{~S}$ [M-H]-: 375.0980, found 375.0981.

IR (thin film) $v_{\max }\left(\mathbf{c m}^{-1}\right): 3214$ (br), 1644 (m), 1557 (s), 1406 (s), 1334 (m), 1135 (s).

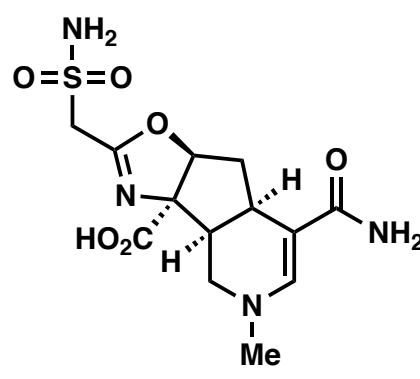

18
TLC: $\mathrm{R}_{\mathrm{f}}=0.3\left(25 \% 7 \mathrm{~N} \mathrm{NH}_{3} /\right.$ Methanol in $\left.\mathrm{DCM}\right)$

${ }^{1} \mathbf{H}$ NMR $\left(700 \mathrm{MHz}, \mathrm{D}_{2} \mathrm{O}\right) \delta 7.38(\mathrm{~s}, 1 \mathrm{H}), 5.44(\mathrm{dd}, J=10.0,2.2 \mathrm{~Hz}$, 1H), $4.39(\mathrm{~d}, J=14.7 \mathrm{~Hz}, 1 \mathrm{H}), 4.37$ (d, $J=14.8 \mathrm{~Hz}, 1 \mathrm{H}), 3.10-$ $2.02(\mathrm{~m}, 2 \mathrm{H}), 2.97(\mathrm{dd}, J=5.9,3.4 \mathrm{~Hz}, 1 \mathrm{H}), 2.83(\mathrm{dt}, J=9.0,3.6$ $\mathrm{Hz}, 1 \mathrm{H}), 2.70$ (s, 3H), 2.58 (ddd, $J=14.3,10.1,5.7 \mathrm{~Hz}, 1 \mathrm{H}), 2.18$ altemicidin oxazoline $(\mathrm{dd}, J=14.3,2.2 \mathrm{~Hz}, 1 \mathrm{H})$. 
${ }^{13}$ C NMR (151 MHz, D $\left.2 \mathrm{O}\right) \delta 173.13,172.32,164.78,141.49,101.00,84.44,67.23,58.07,45.41$, 41.86, 38.68, 35.64, 33.46.

HRMS (m/z): (ESI) calcd. for $\mathrm{C}_{13} \mathrm{H}_{19} \mathrm{~N}_{4} \mathrm{O}_{6} \mathrm{~S}[\mathrm{M}+\mathrm{H}]^{+}: 359.1020$, found 359.1021.

LCMS Trace: 2-90\% MeOH/50mM NH${ }_{4} \mathrm{Oac}$, Luna C18 $100 \AA$ Å column

$\underbrace{5}$

Retention time: compound

4.605: ethylpyridinium iodide

7.415: impurity

7.736: altemicidin oxazoline (18)

11.805: ethyl altemicidin oxazoline (17) 
Table 1 Procedures and Characterization Data:
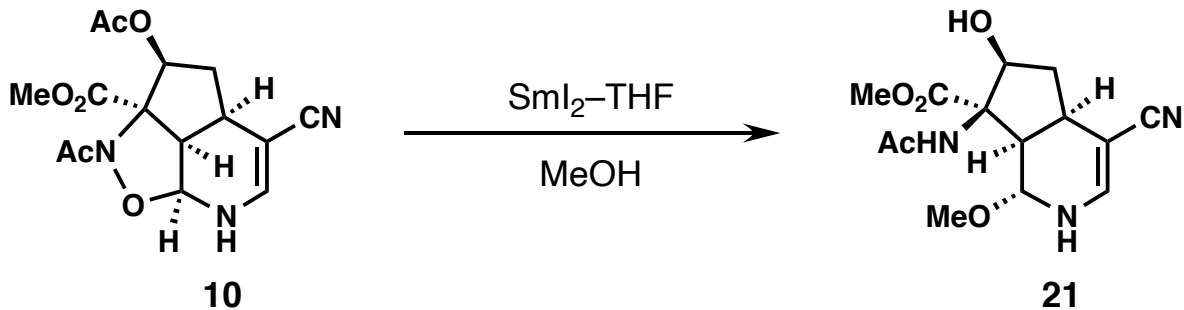

Aminal 18: 21 (4 mg, $0.01 \mathrm{mmol}, 1.0$ equiv.) was suspended in methanol (200 $\mu \mathrm{L})$, equipped with a stir bar, and degassed thoroughly with argon. To this was added a freshly made samarium diiodide solution in $\mathrm{THF}^{2}$ ( 1.6 of a $\sim 0.05 \mathrm{M}$ solution, $0.08 \mathrm{mmol}, 8.0$ equiv.) and the reaction was allowed to stir at room temperature until consumption of the samarium diiodide as indicated by a change from a deep blue mixture to a pale purple solution. The reaction was diluted with methanol and concentrated. Purification by preparative TLC with $6 \% \mathrm{MeOH} / 3: 1 \mathrm{DCM} / \mathrm{Hexanes}$ to give pure compound 21.

TLC: $\mathrm{R}_{\mathrm{f}}=0.4(10 \% \mathrm{MeOH}, 4: 1 \mathrm{DCM} / \mathrm{Hex})$.

${ }^{1}$ H NMR $(700 \mathrm{MHz}, \mathrm{MeOD}) \delta 6.82(\mathrm{~d}, J=1.5 \mathrm{~Hz}, 1 \mathrm{H}), 4.71(\mathrm{~d}, J=1.8 \mathrm{~Hz}, 1 \mathrm{H}), 4.25-4.14(\mathrm{~m}$, 1H), $3.69(\mathrm{~s}, 3 \mathrm{H}), 3.27(\mathrm{~s}, 3 \mathrm{H}), 3.05(\mathrm{dd}, J=9.3,1.9 \mathrm{~Hz}, 1 \mathrm{H}), 2.83(\mathrm{tdd}, J=9.1,4.4,1.4 \mathrm{~Hz}, 1 \mathrm{H})$, $2.48(\mathrm{dt}, J=13.9,8.8 \mathrm{~Hz}, 1 \mathrm{H}), 1.94(\mathrm{~s}, 3 \mathrm{H}), 1.88(\mathrm{ddd}, J=13.9,7.9,4.4 \mathrm{~Hz}, 1 \mathrm{H})$.

${ }^{13}$ C NMR (151 MHz, MeOD) $\delta 174.1,174.0,143.1,123.2,82.3,80.3,76.6,67.8,54.3,52.9,45.0$, $37.9,29.1,22.4$.

IR (thin film) $v_{\max }\left(\mathbf{c m}^{-1}\right)$ : 3328 (br), 2925(m), 2190 (m), 1729 (m), 1639 (s), 1513 (m), 1376 (w), $1268(\mathrm{~m}), 1083(\mathrm{~m}), 1063(\mathrm{~m})$.

HRMS (m/z): (ESI) calcd. for $\mathrm{C}_{14} \mathrm{H}_{19} \mathrm{~N}_{3} \mathrm{O}_{5} \mathrm{Na}[\mathrm{M}+\mathrm{Na}]^{+}:$332.1217, found 332.1216. 


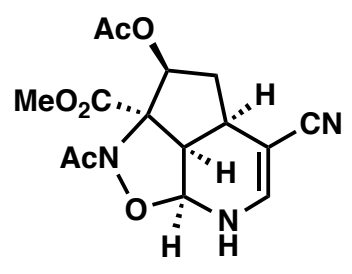

10

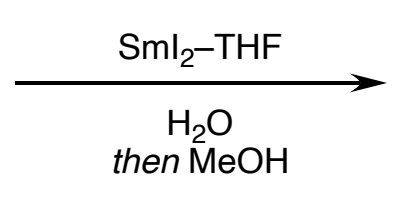

then $\mathrm{MeOH}$

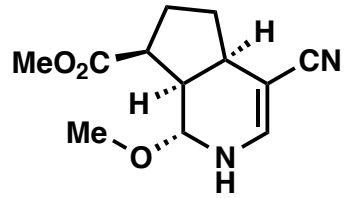

22

22: 10 (4mg, $0.01 \mathrm{mmol}, 1.0$ equiv.) was suspended in water $(200 \mu \mathrm{L})$, equipped with a stir bar, and degassed thoroughly with argon. To this was added a freshly made samarium diiodide solution in THF (1.6 of a $\sim 0.05 \mathrm{M}$ solution, $0.08 \mathrm{mmol}, 8.0$ equiv.) and the reaction was allowed to stir at room temperature until consumption of the samarium diiodide as indicated by a change from a deep purple mixture to a pale purple solution. The reaction was diluted with methanol and concentrated. Purification by preparative TLC with $6 \% \mathrm{MeOH} / 3: 1 \mathrm{DCM} /$ Hexanes to give pure compound 22.

TLC: $\mathrm{R}_{\mathrm{f}}=0.9$ (10\% Methanol in 3:1 DCM/Hexanes).

${ }^{1}$ H NMR (700 MHz, MeOD) $\delta 6.93(\mathrm{~s}, 1 \mathrm{H}), 4.38(\mathrm{~s}, 1 \mathrm{H}), 3.69(\mathrm{~s}, 3 \mathrm{H}), 3.31(\mathrm{~s}, 3 \mathrm{H}) *, 2.87(\mathrm{~s}, 1 \mathrm{H})$, $2.53(\mathrm{dd}, J=15.2,4.9 \mathrm{~Hz}, 1 \mathrm{H}), 2.53-2.46(\mathrm{~m}, 1 \mathrm{H}), 2.04-1.93(\mathrm{~m}, 2 \mathrm{H}), 1.93-1.87(\mathrm{~m}, 2 \mathrm{H})$. *underneath solvent peak, see HSQC

${ }^{13}$ C NMR (151 MHz, MeOD) $\delta$ 177.4, 144.1, 122.4, 82.3, 80.7, 54.7, 52.5, 46.6, 45.5, 35.3, 30.1, 27.4.

HRMS (m/z): (ESI) calcd. for $\mathrm{C}_{12} \mathrm{H}_{16} \mathrm{~N}_{2} \mathrm{O}_{3} \mathrm{Na}[\mathrm{M}+\mathrm{Na}]^{+}: 259.1059$, found 249.1056.
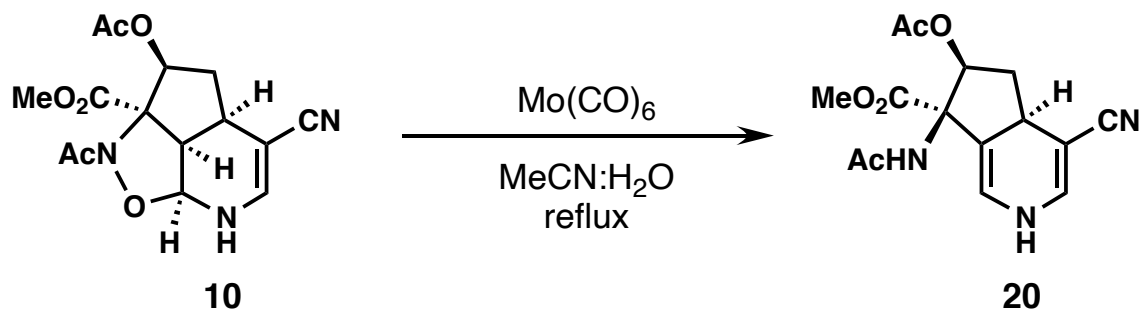
Dihydropyridine 17: 10 (3.4 mg, $0.01 \mathrm{mmol}, 1.0$ equiv.) was combined with molybdenum hexacarbonyl ( $2.6 \mathrm{mg}, 0.01 \mathrm{mmol}, 1.0$ equiv.) in a reaction tube equipped with a stir bar and backfilled with nitrogen to this was added a 9:1 mixture of $\mathrm{MeCN} / \mathrm{H}_{2} \mathrm{O}(0.55 \mathrm{~mL})$ which had been degassed by sparging with argon and concomitant sonication. The reaction mixture was heated to $85{ }^{\circ} \mathrm{C}$ for $16 \mathrm{~h}$. Upon cooling to room temperature the reaction mixture was filtered through Celite ${ }^{\circledR}$, concentrated, and passed through a plug of silica with $10 \% \mathrm{MeOH} / \mathrm{DCM}$ to remove molybdenum byproducts. Further purification by preparative TLC (10\% $\mathrm{MeOH}$ in 3:1 DCM/Hexanes) provided $\mathbf{2 0}$ as the exclusive product of the reaction.

TLC: $\mathrm{R}_{\mathrm{f}}=0.4(10 \%$ Methanol in 3:1 DCM/Hexanes).

${ }^{1}$ H NMR (600 MHz, MeOD) $\delta 6.86(\mathrm{~s}, 1 \mathrm{H}), 6.34(\mathrm{~d}, J=2.2 \mathrm{~Hz}, 1 \mathrm{H}), 5.83(\mathrm{t}, J=6.8 \mathrm{~Hz}, 1 \mathrm{H})$, $3.68(\mathrm{~s}, 3 \mathrm{H}), 3.33(\mathrm{q}, J=2.0 \mathrm{~Hz}, 1 \mathrm{H}), 2.64(\mathrm{dt}, J=12.6,7.1 \mathrm{~Hz}, 1 \mathrm{H}), 1.99(\mathrm{~s}, 3 \mathrm{H}), 1.97(\mathrm{~s}, 3 \mathrm{H})$, $1.83(\mathrm{ddd}, J=12.6,9.5,6.9 \mathrm{~Hz}, 1 \mathrm{H})$.

${ }^{13}$ C NMR (151 MHz, MeOD) $\delta$ 173.2, 171.7, 171.5, 141.1, 123.4, 121.3, 113.6, 80.1, 73.1, 67.9, 53.2, 39.0, 33.2, 22.0, 20.8 .

HRMS (m/z): (ESI) calcd. for $\mathrm{C}_{15} \mathrm{H}_{17} \mathrm{~N}_{3} \mathrm{O}_{5} \mathrm{Na}[\mathrm{M}+\mathrm{Na}]^{+}:$342.4060, found 342.1061.

IR (thin film) $v_{\max }\left(\mathbf{c m}^{-1}\right)$ : 3352 (br), 2955 (w), 2928 (w), 2199 (m), 1736 (s), 1662 (m), 1496 (m), 1237 (s).

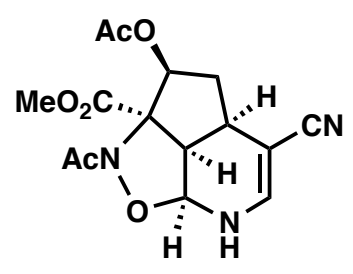

10

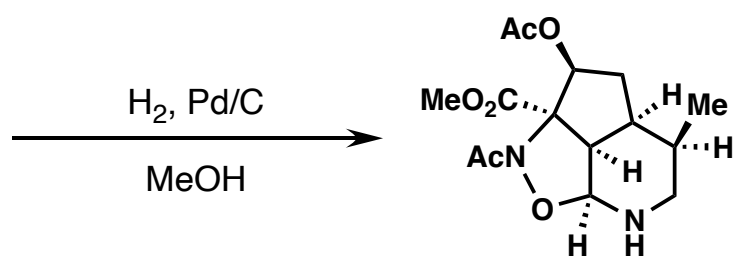

23

Piperidine 20: 10 (3.4 mg, $0.01 \mathrm{mmol}, 1.0$ equiv.) was combined with 10\% $\mathrm{Pd} / \mathrm{C}$ and suspended in methanol $(1 \mathrm{~mL})$ in a reaction tube equipped with a stir bar. The tube was sealed with a septum and equipped with a hydrogen balloon which was engaged via an 18-gauge needle. The reaction 
mixture was sparged with hydrogen for 10 minutes and the mixture was allowed to stir $12 \mathrm{~h}$ under positive hydrogen pressure. Upon reaction completion the mixture was filtered through Celite ${ }^{\circledR}$ with methanol and concentrated in vacuo. The crude mixture was further purified by preparatory TLC (8\% Methanol in 3:1 DCM/Hexanes) to yield 23 as the main product of the reaction.

TLC: $\mathrm{R}_{\mathrm{f}}=0.7$ (10\% Methanol in 3:1 DCM/Hexanes).

${ }^{1}$ H NMR (600 MHz, MeOD) $\delta 5.76(\mathrm{dd}, J=11.6,6.5 \mathrm{~Hz}, 1 \mathrm{H}), 5.24(\mathrm{~d}, J=5.3 \mathrm{~Hz}, 1 \mathrm{H}), 3.72(\mathrm{~s}$, $3 \mathrm{H}), 2.93(\mathrm{dd}, J=8.8,5.4 \mathrm{~Hz}, 1 \mathrm{H}), 2.80(\mathrm{t}, J=11.7 \mathrm{~Hz}, 1 \mathrm{H}), 2.51(\mathrm{dd}, J=11.7,4.2 \mathrm{~Hz}, 1 \mathrm{H})$, $2.26(\mathrm{ddt}, J=14.3,8.8,5.7 \mathrm{~Hz}, 1 \mathrm{H}), 2.09(\mathrm{~s}, 3 \mathrm{H}), 2.01(\mathrm{dt}, J=12.2,6.3 \mathrm{~Hz}, 1 \mathrm{H}), 1.97(\mathrm{~s}, 3 \mathrm{H})$, $1.94(\mathrm{~m}, 1 \mathrm{H}), 1.77(\mathrm{dt}, J=13.9,11.5 \mathrm{~Hz}, 1 \mathrm{H}), 0.92(\mathrm{~d}, J=7.0 \mathrm{~Hz}, 3 \mathrm{H})$.

${ }^{13}$ C NMR (151 MHz, MeOD) $\delta 171.9,170.1,168.4,91.9,75.9,75.3,53.3,51.9,49.4,40.6,36.3$, $30.5,30.4,20.8,20.5,17.2$

HRMS (m/z): (ESI) calcd. for $\mathrm{C}_{15} \mathrm{H}_{22} \mathrm{~N}_{2} \mathrm{O}_{6} \mathrm{Na}[\mathrm{M}+\mathrm{Na}]^{+}: 349.1370$, found 349.1370.

IR (thin film) $v_{\max }\left(\mathbf{c m}^{-1}\right)$ : 3371 (br), 2957 (w), 2877 (w), 1745 (s), 1655 (s), 1435 (w), 1236 (s).
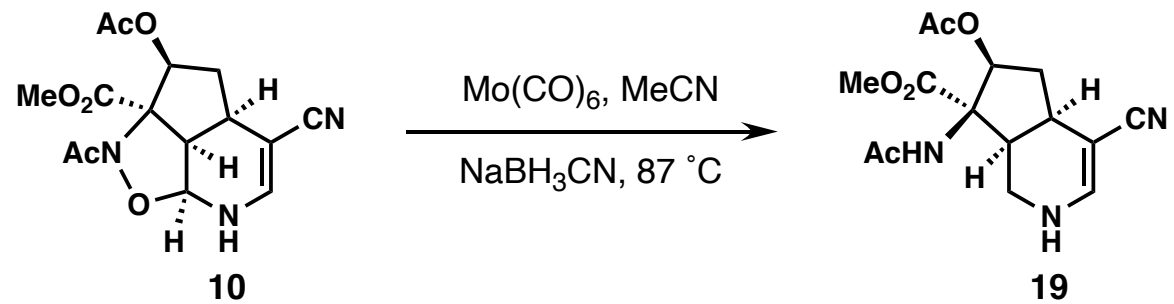

19: Prepared in analogous fashion to $\mathbf{1 2 .}$

TLC: $\mathrm{R}_{\mathrm{f}}: 0.55$ (15\% MeOH in 2:1 DCM/hexanes).

${ }^{1}$ H NMR (700 MHz, MeOD) $\delta 7.08(\mathrm{~s}, 1 \mathrm{H}), 5.44(\mathrm{t}, J=7.8,6.4 \mathrm{~Hz}, 1 \mathrm{H}), 3.69$ (s, 3H), 3.23 (ddd, $J=12.9,5.2,1.4 \mathrm{~Hz}, 1 \mathrm{H}), 3.13(\mathrm{dd}, J=12.9,8.8 \mathrm{~Hz}, 1 \mathrm{H}), 2.83-2.73(\mathrm{~m}, 2 \mathrm{H}), 2.67(\mathrm{dt}, J=14.0$, $7.8 \mathrm{~Hz}, 1 \mathrm{H}), 2.06$ (s, 3H), $2.00(\mathrm{~s}, 3 \mathrm{H}), 1.67$ (ddd, $J=14.0,8.7,6.4 \mathrm{~Hz}, 1 \mathrm{H})$. 
${ }^{13}$ C NMR (176 MHz, MeOD) $\delta$ 173.9, 173.8, 171.1, 146.8, 124.0, 76.8, 75.8, 68.9, 53.2, 41.6, $39.8,38.3,33.9,21.9,20.8$.

HRMS (m/z): (ESI) calcd. for $\mathrm{C}_{15} \mathrm{H}_{19} \mathrm{~N}_{3} \mathrm{O}_{5} \mathrm{Na}[\mathrm{M}+\mathrm{Na}]^{+}:$344.1217, found 344.1215.

IR (thin film) $v_{\max }\left(\mathbf{c m}^{-1}\right): 3366$ (br), 2954 (w), 2928 (w), 2855 (w), 2186 (s), 1734 (s), 1626 (s), $1506(\mathrm{~s}), 1235(\mathrm{~s})$. 


\section{Natural Product Spectral Comparisons}

Altemicidin ${ }^{1} \mathrm{H}$ NMR Spectra Comparison:
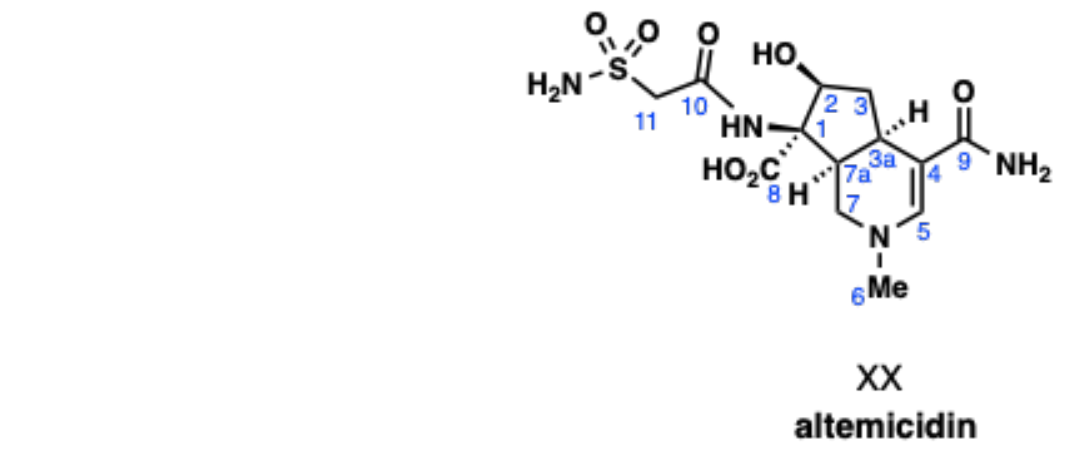

\begin{tabular}{|c|c|c|}
\hline Position & $\begin{array}{c}{ }^{1} \mathrm{H}(\delta) \\
\text { Natural Sample } \\
\left(400 \mathrm{MHz}, \mathrm{D}_{2} \mathrm{O}\right)[\text { ref. } 2]^{3}\end{array}$ & $\begin{array}{c}{ }^{1} \mathrm{H}(\delta) \\
\text { Synthetic Sample } \\
\left(600 \mathrm{MHz}, \mathrm{D}_{2} \mathrm{O}\right)\end{array}$ \\
\hline $2-\mathrm{H}$ & $4.28(\mathrm{dd}, J=7.6,9.0 \mathrm{~Hz}, 1 \mathrm{H})$ & $4.28(\mathrm{~m}, 1 \mathrm{H})$ \\
\hline $3-\mathrm{H}_{\mathrm{a}}$ & $1.26(\mathrm{dt}, J=9.0,12.6 \mathrm{~Hz}, 1 \mathrm{H})$ & $1.26(\mathrm{dt}, J=8.8,12.8 \mathrm{~Hz}, 1 \mathrm{H})$ \\
\hline $3-\mathrm{H}_{\mathrm{b}}$ & $2.67(\mathrm{dt}, J=7.6,12.6 \mathrm{~Hz}, 1 \mathrm{H})$ & $2.67(\mathrm{~m}, 1 \mathrm{H})$ \\
\hline $3 \mathrm{a}-\mathrm{H}$ & $\sim 2.93(\mathrm{~m})$ & $7.39(\mathrm{~s}, 1 \mathrm{H})$ \\
\hline $5-\mathrm{H}$ & $7.39(\mathrm{~s}, 1 \mathrm{H})$ & $2.98(\mathrm{~s}, 3 \mathrm{H})$ \\
\hline $6-\mathrm{H}$ & $2.98(\mathrm{~s}, 3 \mathrm{H})$ & $2.89-2.81(\mathrm{~m})$ \\
\hline $7-\mathrm{H}_{\mathrm{a}}$ & $\sim 2.86(\mathrm{~m})$ & $2.89-2.81(\mathrm{~m})$ \\
\hline $7-\mathrm{H}_{\mathrm{b}}$ & $\sim 2.86(\mathrm{~m})$ & $2.89-2.81(\mathrm{~m})$ \\
\hline $7 \mathrm{a}-\mathrm{H}$ & $\sim 2.86(\mathrm{~m})$ & $4.29(\mathrm{~d}, J=14.1 \mathrm{~Hz}, 1 \mathrm{H})$ \\
\hline $11-\mathrm{H}_{\mathrm{a}}$ & $4.29(\mathrm{~d}, J=14.0 \mathrm{~Hz}, 1 \mathrm{H})$ & $4.38(\mathrm{~d}, J=14.1 \mathrm{~Hz}, 1 \mathrm{H})$ \\
\hline $11-\mathrm{H}_{\mathrm{b}}$ & $4.38(\mathrm{~d}, J=14.0 \mathrm{~Hz}, 1 \mathrm{H})$ & $\mathrm{r})$ \\
\hline
\end{tabular}


Altemicidin ${ }^{13} \mathrm{C}$ NMR Spectra Comparison:

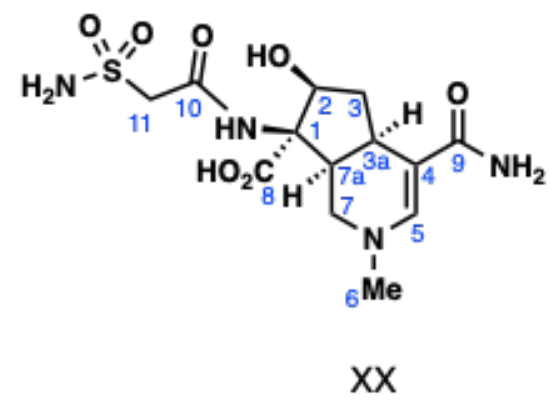

altemicidin

\begin{tabular}{|c|c|c|}
\hline Position & $\begin{array}{c}{ }^{13} \mathrm{C}(\delta) \\
\text { Natural Sample } \\
\left(100 \mathrm{MHz}, \mathrm{D}_{2} \mathrm{O}\right)[\text { ref. } 2]\end{array}$ & $\begin{array}{c}{ }^{13} \mathrm{C}(\delta) \\
\text { Synthetic Sample } \\
\left(151 \mathrm{MHz}, \mathrm{D}_{2} \mathrm{O}\right)\end{array}$ \\
\hline 1 & 69.1 & 69.1 \\
\hline 2 & 76.0 & 76.0 \\
\hline 3 & 41.4 & 41.3 \\
\hline $3 \mathrm{a}$ & 31.7 & 31.7 \\
\hline 4 & 97.1 & 97.1 \\
\hline 5 & 147.3 & 447.3 \\
\hline 6 & 43.2 & 43.2 \\
\hline 7 & 45.5 & 45.5 \\
\hline $7 \mathrm{a}$ & 40.8 & 40.8 \\
\hline 8 & 179.7 & 179.7 \\
\hline 9 & 174.2 & 164.4 \\
\hline 10 & 164.4 & 60.1 \\
\hline 11 & 60.3 & \\
\hline & & 174.2 \\
\hline
\end{tabular}




\section{ESP Surface Data}
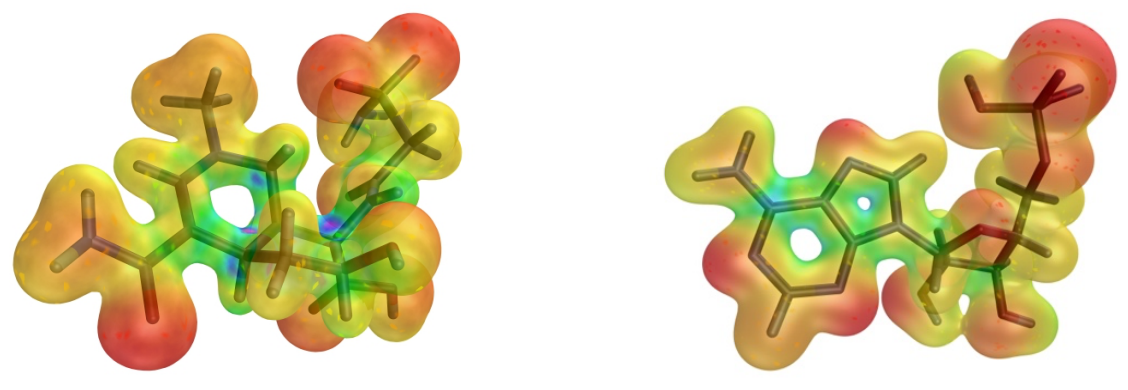

ESP altemicidin (1)

ESP AMP (4)

Electrostatic potential (ESP) surfaces for altemicidin (1) and AMP (4) were generated by Macromodel minimization of the 3D structure in Maestro, ${ }^{4}$ followed by optimization and frequency calculations in Gaussian $16^{5}(\mathrm{~B} 3 \mathrm{LYP} / 6-31+\mathrm{g}(\mathrm{d}))$. The ESP surfaces were visualized with IQmol ${ }^{6}$ (isovalue $=0.05$, rainbow color gradient -0.1054 (red) to 0.4041 (purple)).

Gaussian title card:

\# opt freq=noraman b3lyp/6-31+g(d) scrf=(iefpcm,solvent=water) geom=connectivity empiricaldispersion $=\mathrm{gd} 3 \mathrm{bj}$ integral $=$ ultrafinegrid

Altemicidin (1) Cartesian coordinates:

\$altemicidin-opt-freq

01

$\begin{array}{llll}\mathrm{C} & -2.5184 & 3.157 & -4.712\end{array}$

$\begin{array}{llll}\text { C } & -2.2758 & 1.9694 & -5.6676\end{array}$

$\begin{array}{llll}\text { C } & -3.5003 & 1.933 & -6.5927\end{array}$

C $\quad-4.1233 \quad 3.4014-6.5484$

$\begin{array}{llll}\text { C } & -3.1883 & 4.223 & -5.6084\end{array}$

$\begin{array}{llll}\text { C } & -2.1277 & 5.0349 & -6.3697\end{array}$

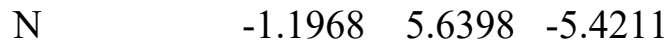

$\begin{array}{llll}\text { C } & -0.7538 & 4.882 & -4.3765\end{array}$

$\begin{array}{lllll}\text { C } & & -1.2938 & 3.6847 & -4.0054\end{array}$

$\begin{array}{llll}\mathrm{O} & -3.1814 & 1.6063 & -7.951\end{array}$

$\begin{array}{llll}\mathrm{N} & -4.2334 & 3.9221 & -7.9048\end{array}$

C $\quad-5.5225 \quad 3.2728 \quad-5.9106$

$\begin{array}{lllll}\mathrm{O} & -5.7734 & 3.4489 & -4.7409\end{array}$

$\begin{array}{llll}\mathrm{O} & -6.4437 & 2.8289 & -6.7998\end{array}$

$\begin{array}{llll}\text { C } & -0.409 & 6.7633 & -5.9075\end{array}$

$\begin{array}{llll}\mathrm{C} & -0.8104 & 2.9109 & -2.8501\end{array}$

$\begin{array}{llll}\mathrm{N} & 0.4082 & 3.2668 & -2.2828\end{array}$ 


\begin{tabular}{llll}
$\mathrm{O}$ & -1.465 & 1.9798 & -2.3686 \\
$\mathrm{C}$ & -4.8817 & 5.0673 & -8.1947 \\
$\mathrm{C}$ & -4.8783 & 5.4805 & -9.6605 \\
$\mathrm{O}$ & -5.4254 & 5.7734 & -7.3383 \\
$\mathrm{~S}$ & -4.1247 & 7.1461 & -9.834 \\
$\mathrm{O}$ & -2.8554 & 7.091 & -9.1034 \\
$\mathrm{O}$ & -4.1859 & 7.5026 & -11.2529 \\
$\mathrm{~N}$ & -5.127 & 8.2059 & -9.0208 \\
$\mathrm{H}$ & -3.2326 & 2.8415 & -3.9451 \\
$\mathrm{H}$ & -1.3875 & 2.1506 & -6.2831 \\
$\mathrm{H}$ & -2.1295 & 1.03 & -5.1278 \\
$\mathrm{H}$ & -4.2511 & 1.2227 & -6.2289 \\
$\mathrm{H}$ & -3.7817 & 4.9148 & -5.0085 \\
$\mathrm{H}$ & -1.5741 & 4.3989 & -7.0799 \\
$\mathrm{H}$ & -2.5836 & 5.8376 & -6.9505 \\
$\mathrm{H}$ & 0.0494 & 5.3363 & -3.8031 \\
$\mathrm{H}$ & -3.0125 & 0.659 & -8.0221 \\
$\mathrm{H}$ & -3.8694 & 3.3263 & -8.6393 \\
$\mathrm{H}$ & -7.2824 & 2.7466 & -6.315 \\
$\mathrm{H}$ & -1.0699 & 7.5254 & -6.3292 \\
$\mathrm{H}$ & 0.1507 & 7.2069 & -5.0803 \\
$\mathrm{H}$ & 0.3022 & 6.4585 & -6.691 \\
$\mathrm{H}$ & 1.1286 & 3.6627 & -2.8684 \\
$\mathrm{H}$ & 0.7343 & 2.6155 & -1.5814 \\
$\mathrm{H}$ & -4.2849 & 4.8292 & -10.3067 \\
$\mathrm{H}$ & -5.8988 & 5.5467 & -10.0452 \\
$\mathrm{H}$ & -5.9415 & 8.4419 & -9.5816 \\
$\mathrm{H}$ & -5.3775 & 7.8345 & -8.1017 \\
Send & & & \\
\hline & & &
\end{tabular}

AMP (4) Cartesian coordinates:

$\begin{array}{lrrr}\text { \$AMP-opt-freq_1 } & & \\ 0 \text { 1 } & & & \\ \text { P } & 3.70172300 & -1.58513900 & -0.19284100 \\ \text { O } & 0.72536200 & 0.89617100 & -0.95742300 \\ \text { O } & -1.07002300 & 3.00024900 & 1.36234100 \\ \text { O } & 0.75114600 & 3.95828100 & -0.34193800 \\ \text { O } & 3.29705900 & -0.13094800 & -0.70659000 \\ \text { O } & 2.37614700 & -2.01802300 & 0.61192800 \\ \text { O } & 4.75191600 & -1.39992400 & 1.02099900 \\ \text { O } & 4.15939300 & -2.45581700 & -1.30418900 \\ \text { N } & -1.29178400 & 0.13679700 & -0.07812400 \\ \text { N } & -1.83881900 & -1.93541800 & 0.61839200 \\ \text { N } & -3.51318500 & 0.92332100 & -0.76098600\end{array}$




$\begin{array}{lrrr}\mathrm{N} & -5.21407000 & -0.75424300 & -0.40910600 \\ \mathrm{~N} & -4.78715100 & -2.84745500 & 0.52040300 \\ \mathrm{C} & -0.07882100 & 2.12064600 & 0.89009600 \\ \mathrm{C} & 1.19652600 & 2.81596300 & 0.38861600 \\ \mathrm{C} & -0.49271000 & 1.31156500 & -0.35776200 \\ \mathrm{C} & 1.81502100 & 1.74597300 & -0.52675700 \\ \mathrm{C} & 2.86112600 & 0.91403700 & 0.19643200 \\ \mathrm{C} & -2.65571000 & 0.01887800 & -0.26247600 \\ \mathrm{C} & -0.86240900 & -1.06773000 & 0.45190800 \\ \mathrm{C} & -2.97466600 & -1.27020300 & 0.18016700 \\ \mathrm{C} & -4.33174400 & -1.64553000 & 0.09197000 \\ \mathrm{C} & -4.76412000 & 0.45061400 & -0.79955100 \\ \mathrm{H} & 0.16072400 & 1.43115800 & 1.70431500 \\ \mathrm{H} & 1.86088200 & 3.11209600 & 1.20560000 \\ \mathrm{H} & -1.08402500 & 1.93522200 & -1.03645800 \\ \mathrm{H} & 2.25545600 & 2.20256200 & -1.41799000 \\ \mathrm{H} & 2.45530000 & 0.47265100 & 1.10996900 \\ \mathrm{H} & 3.72900000 & 1.53046900 & 0.44648600 \\ \mathrm{H} & -1.01605700 & 3.80532000 & 0.81313700 \\ \mathrm{H} & 1.47472700 & 4.59874100 & -0.42356500 \\ \mathrm{H} & 0.18269300 & -1.25452000 & 0.65436400 \\ \mathrm{H} & -5.51806600 & 1.12486000 & -1.19793600 \\ \mathrm{H} & -4.12429100 & -3.58566100 & 0.71539600 \\ \mathrm{H} & -5.72655600 & -3.12395900 & 0.26637100 \\ \mathrm{H} & 2.45636100 & -2.86079500 & 1.09371400 \\ \mathrm{H} & 5.68012700 & -1.43726500 & 0.72828200 \\ \mathrm{Send} & & & \end{array}$




\section{References}

(1) Wolckenhauer, S. A.; Devlin, A. S.; Du Bois, J. $\delta$-Sultone Formation Through Rh-Catalyzed C-H Insertion. Org. Lett. 2007, 9 (21), 4363.

(2) Szostak, M.; Spain, M.; Procter, D. J. Preparation of Samarium(II) Iodide: Quantitative Evaluation of the Effect of Water, Oxygen, and Peroxide Content, Preparative Methods, and the Activation of Samarium Metal. J. Org. Chem. 2012, 77 (7), 3049.

(3) Takahashi, A.; Ikeda, D.; Nakamura, H.; Naganawa, H.; Kurasawa, S.; Okami, Y.; Takeuchi, T. Altemicidin, A New Acaricidal and Antitumor Substance II. Structural Determination. $J$. Antibiot. (Tokyo) 1989, 42, 1562.

(4) Schrödinger Release 2021-1: Maestro, Schrödinger, LLC, New York, NY, 2021.

(5) Frisch, M. J.; Trucks, G. W.; Schlegel, H. B.; Scuseria, G. E.; Robb, M. A.; Cheeseman, J. R.; Scalmani, G.; Barone, V.; Petersson, G. A.; Nakatsuji, H.; Li, X.; Caricato, M.; Marenich, A. V.; Bloino, J.; Janesko, B. G.; Gomperts, R.; Mennucci, B.; Hratchian, H. P.; Ortiz, J. V.; Izmaylov, A. F.; Sonnenberg, J. L.; Williams; Ding, F.; Lipparini, F.; Egidi, F.; Goings, J.; Peng, B.; Petrone, A.; Henderson, T.; Ranasinghe, D.; Zakrzewski, V. G.; Gao, J.; Rega, N.; Zheng, G.; Liang, W.; Hada, M.; Ehara, M.; Toyota, K.; Fukuda, R.; Hasegawa, J.; Ishida, M.; Nakajima, T.; Honda, Y.; Kitao, O.; Nakai, H.; Vreven, T.; Throssell, K.; Montgomery Jr., J. A.; Peralta, J. E.; Ogliaro, F.; Bearpark, M. J.; Heyd, J. J.; Brothers, E. N.; Kudin, K. N.; Staroverov, V. N.; Keith, T. A.; Kobayashi, R.; Normand, J.; Raghavachari, K.; Rendell, A. P.; Burant, J. C.; Iyengar, S. S.; Tomasi, J.; Cossi, M.; Millam, J. M.; Klene, M.; Adamo, C.; Cammi, R.; Ochterski, J. W.; Martin, R. L.; Morokuma, K.; Farkas, O.; Foresman, J. B.; Fox, D. J. Gaussian 16 Rev. C.01; Wallingford, CT, 2016.

(6) Gilbert, A. IQMol; 2020. 


\section{Appendix A: Ammonia Methanol Solution}

A standard 4L glass bottle of $\mathrm{MeOH}$ was clamped securely in a well ventilated hood and cooled to 0 to 10 ${ }^{\circ} \mathrm{C}$ in a large ice-water bath, with the bath covering the entire body of the bottle. A tank of ammonia gas was appropriately secured in the hood and equipped with a standard regulator. An outlet hose connected to a 9 inch glass pipet was inserted into the $\mathrm{MeOH}$ and attached to the regulator as shown in SI-Figure 1. Ammonia gas was bubbled into the $\mathrm{MeOH}$ in a steady stream until saturation

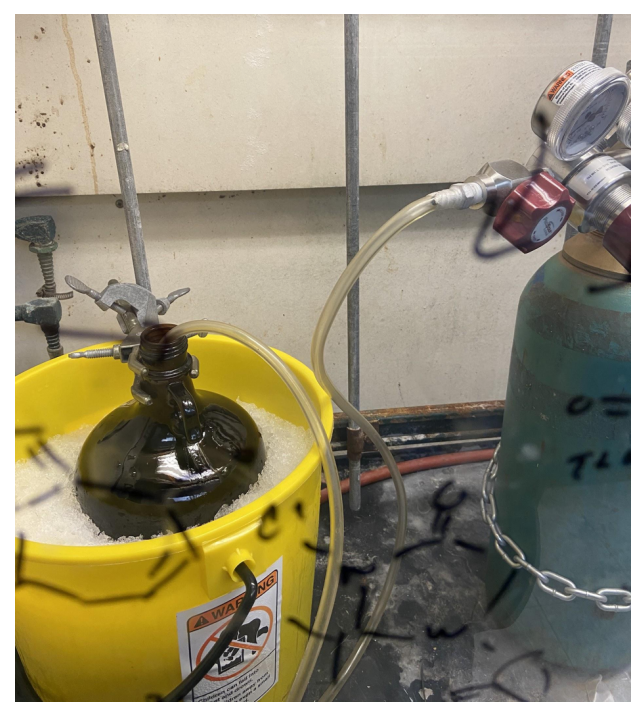

SI-Figure 1: Ammonia gas is bubbled into a cooled $4 \mathrm{~L}$ of $\mathrm{MeOH}$. was reached as indicated by visible bubbling of the solution. Care was taken to maintain the ice bath replenished as the process is somewhat exothermic. The resulting solution was stored at $4{ }^{\circ} \mathrm{C}$ when not in use. 
Appendix B: NMR Spectra

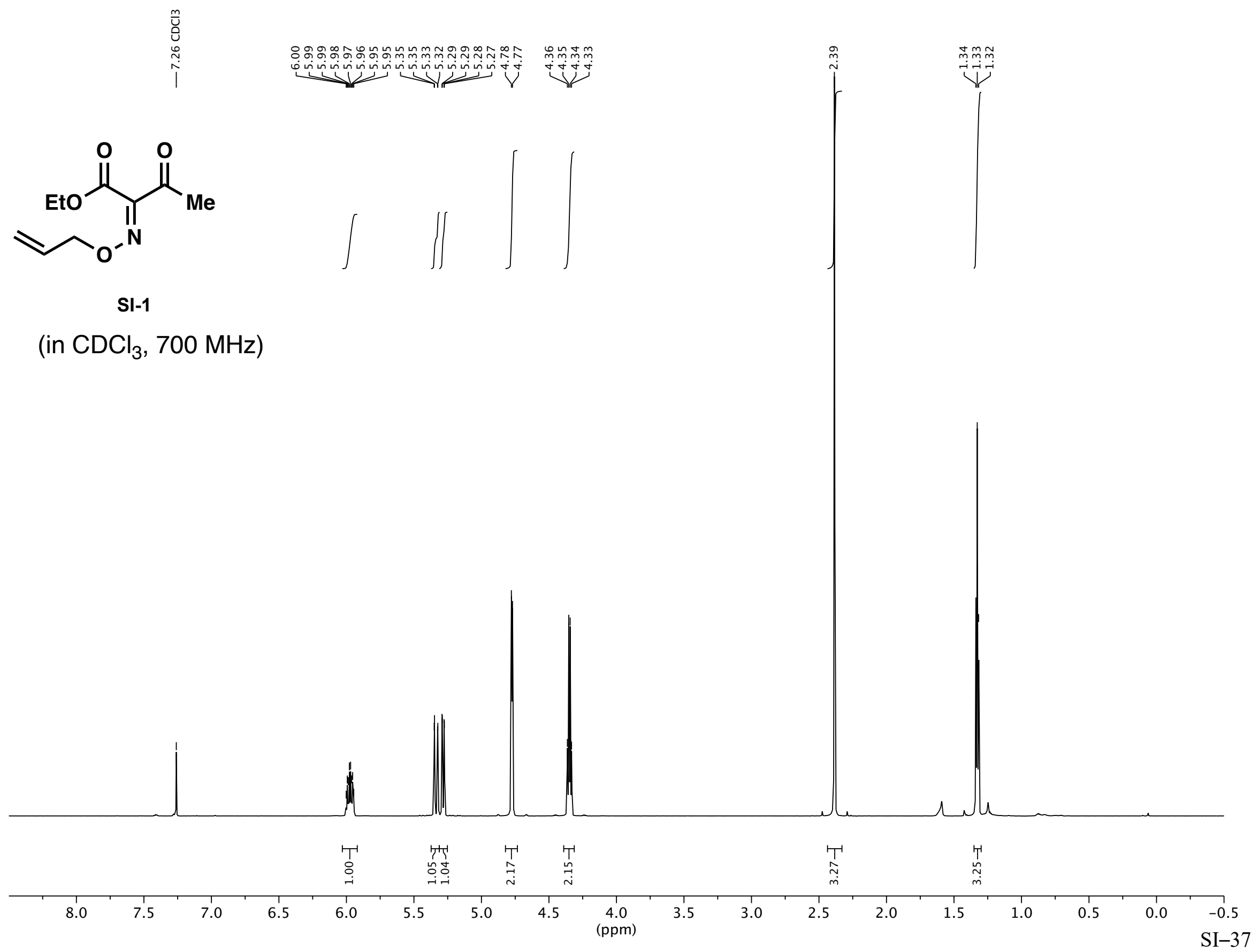




$$
\pm 1
$$




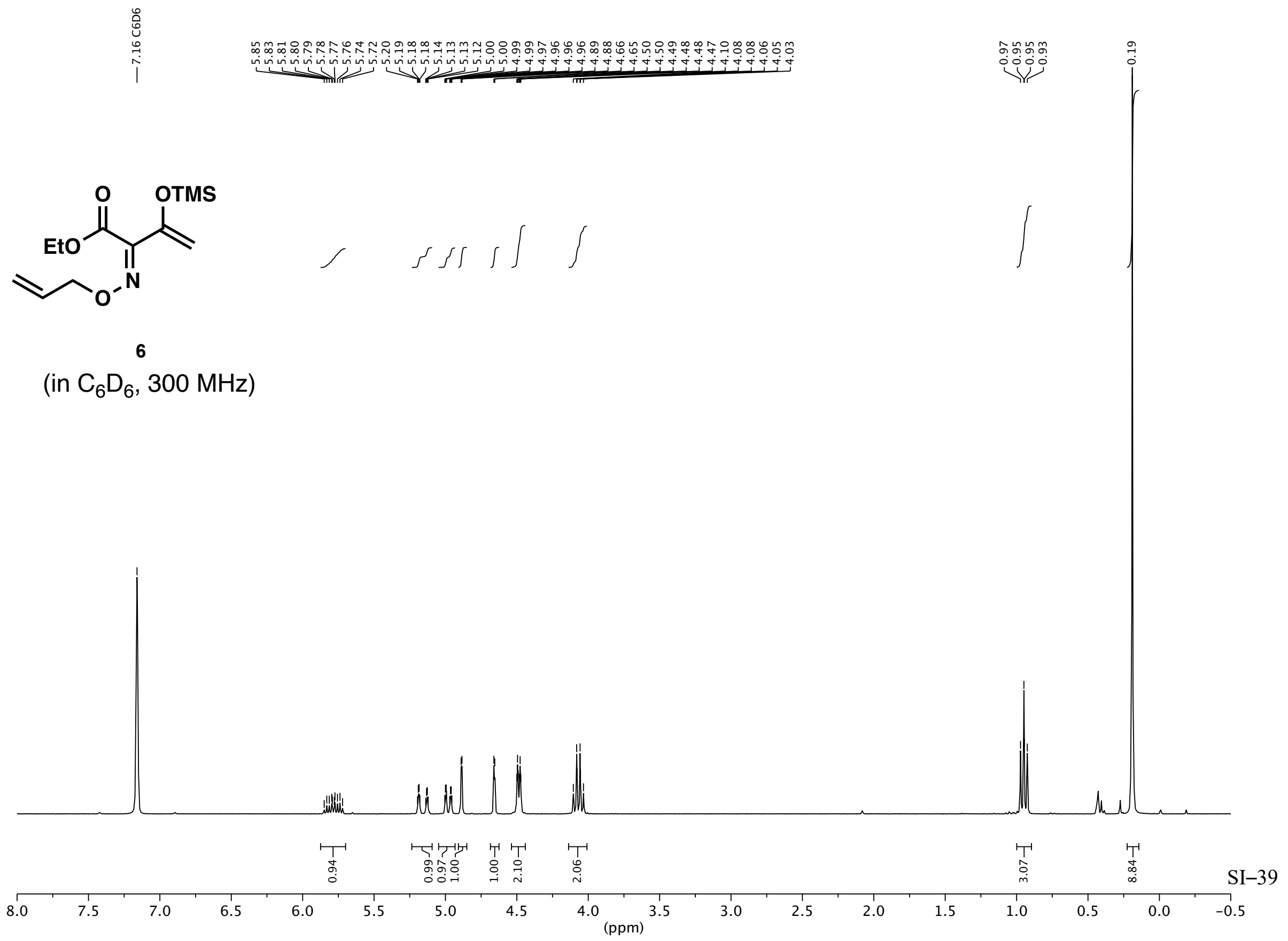



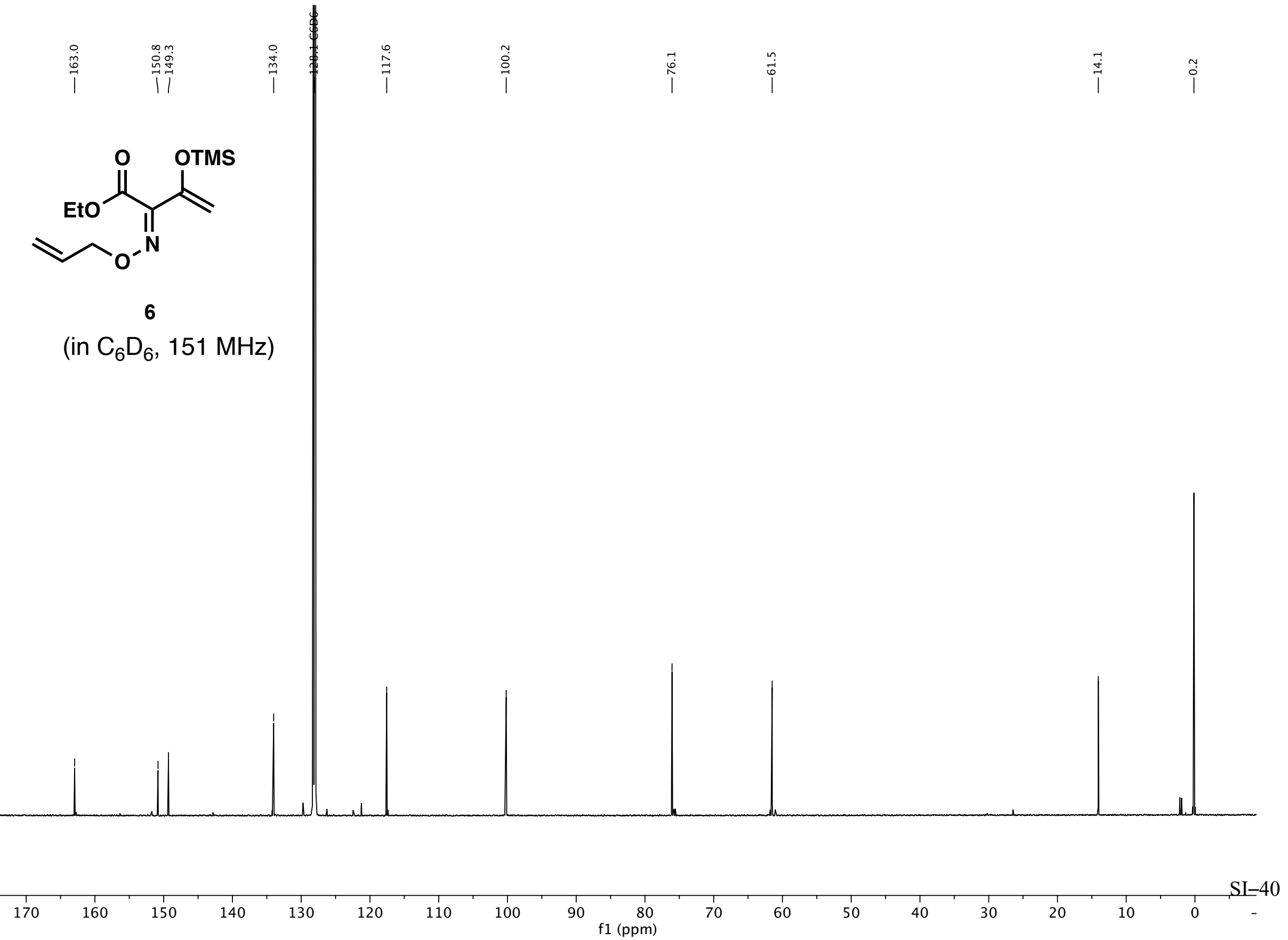
$\stackrel{\circ}{\circ}$

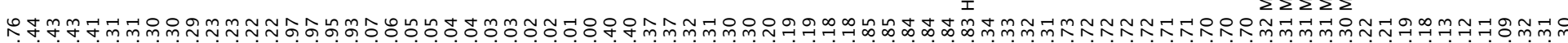

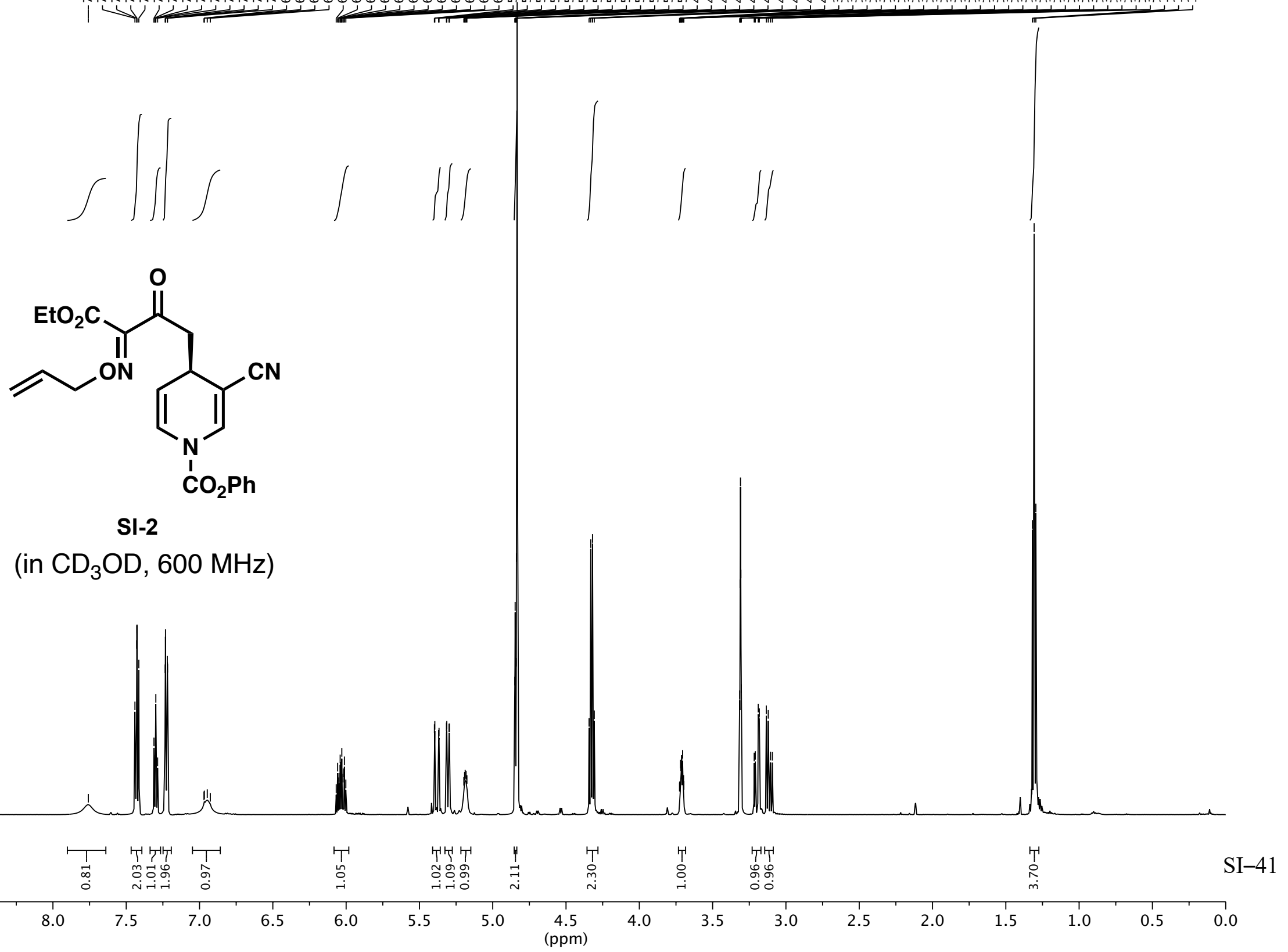




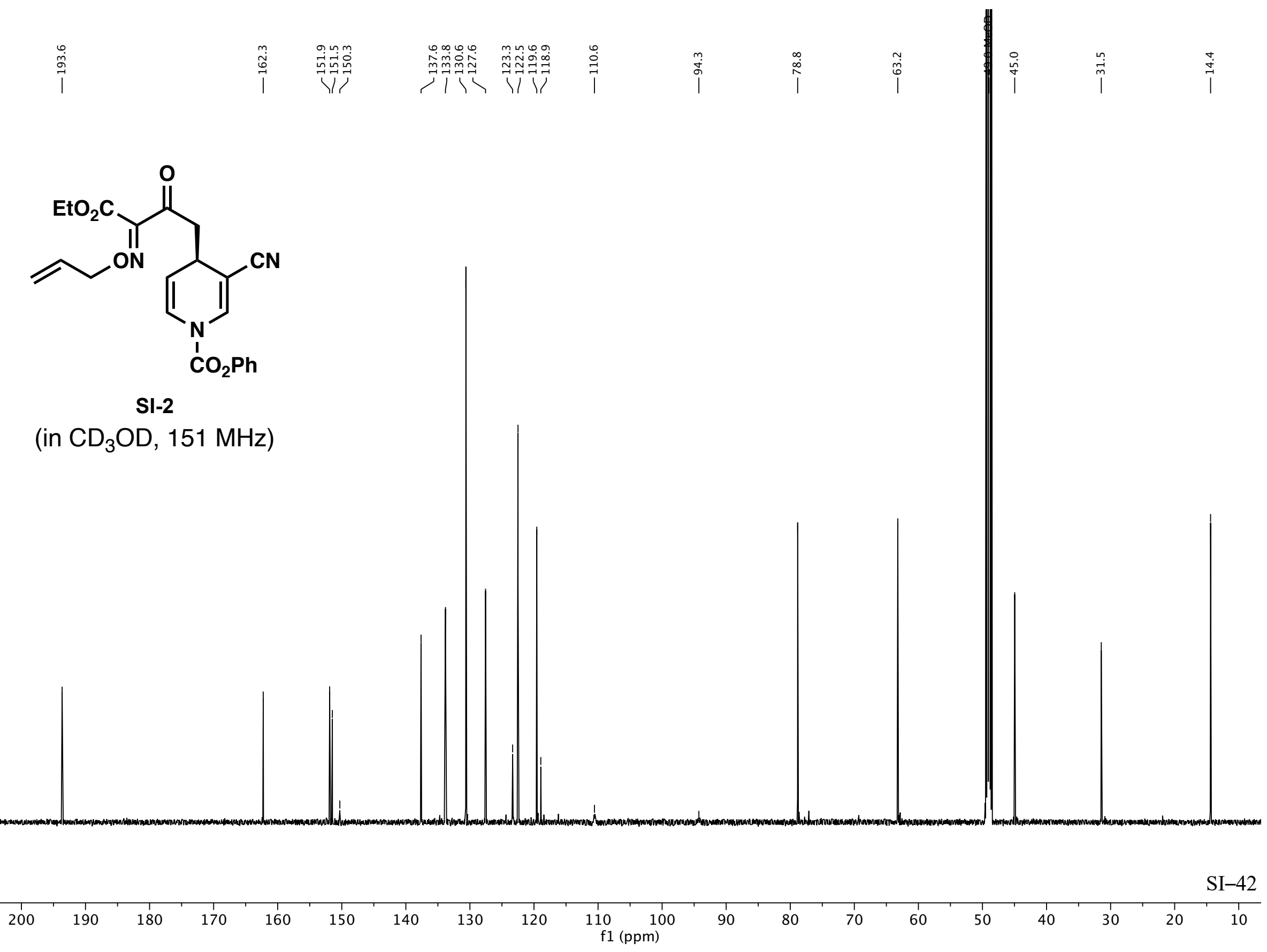




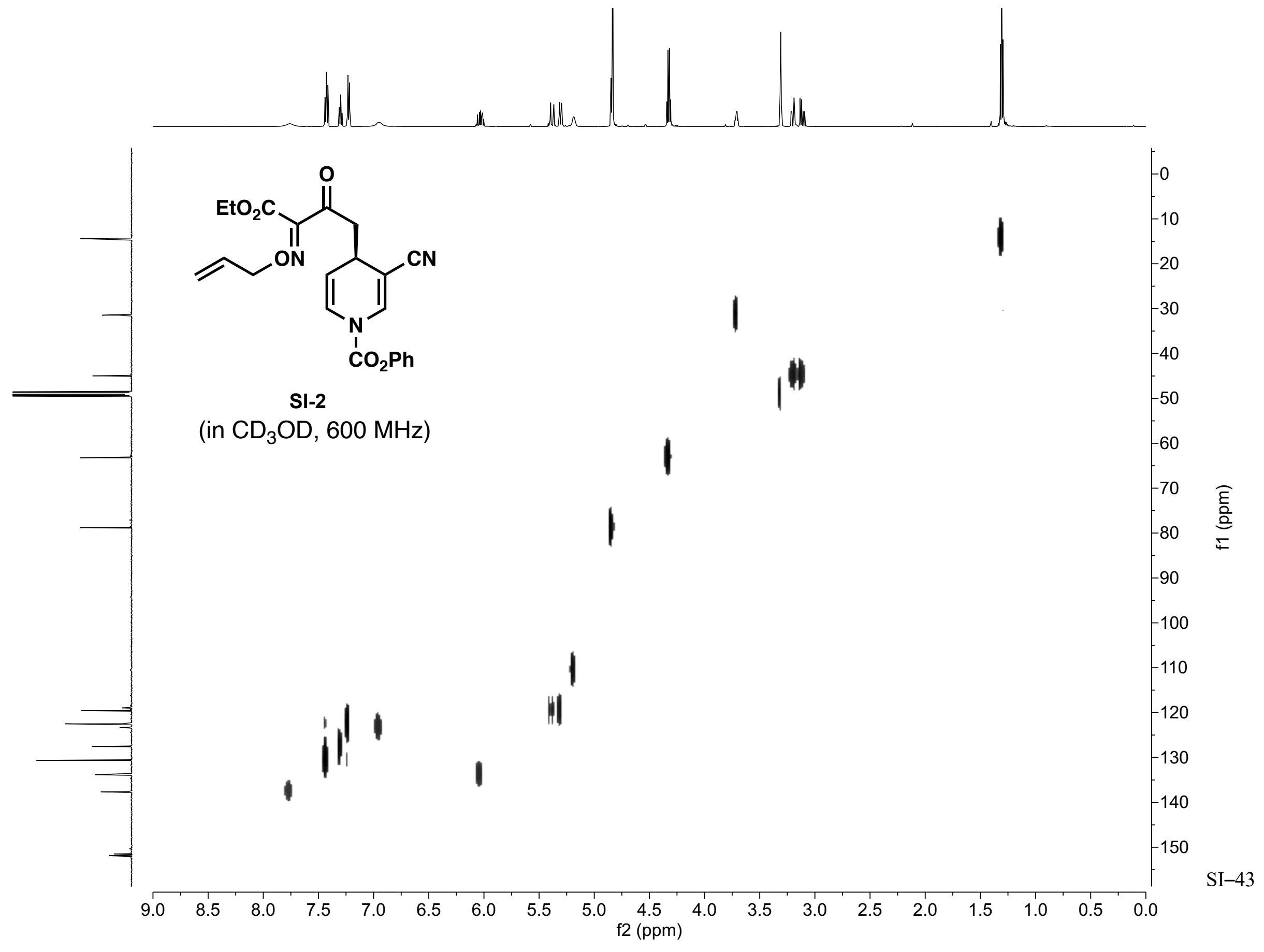




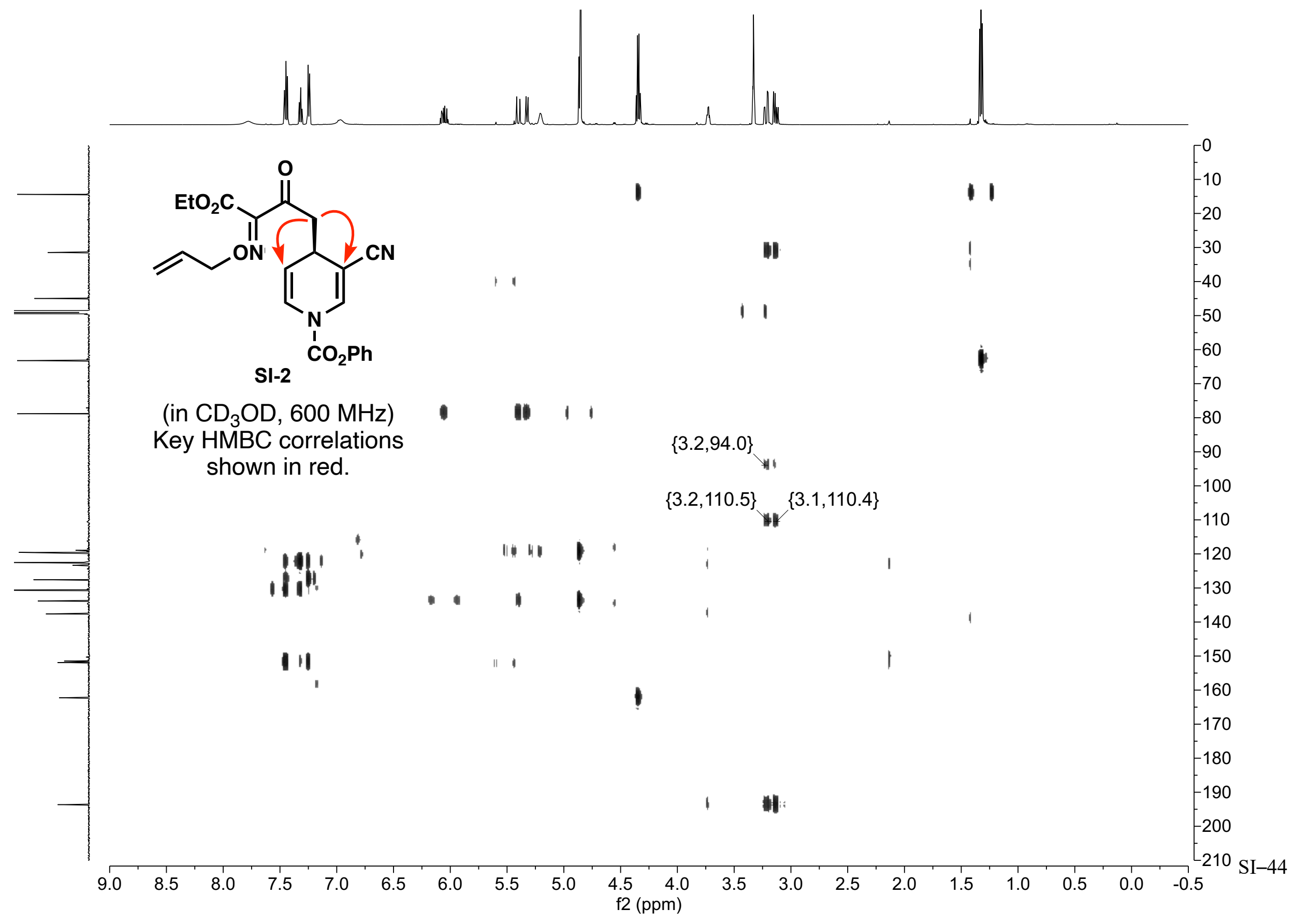




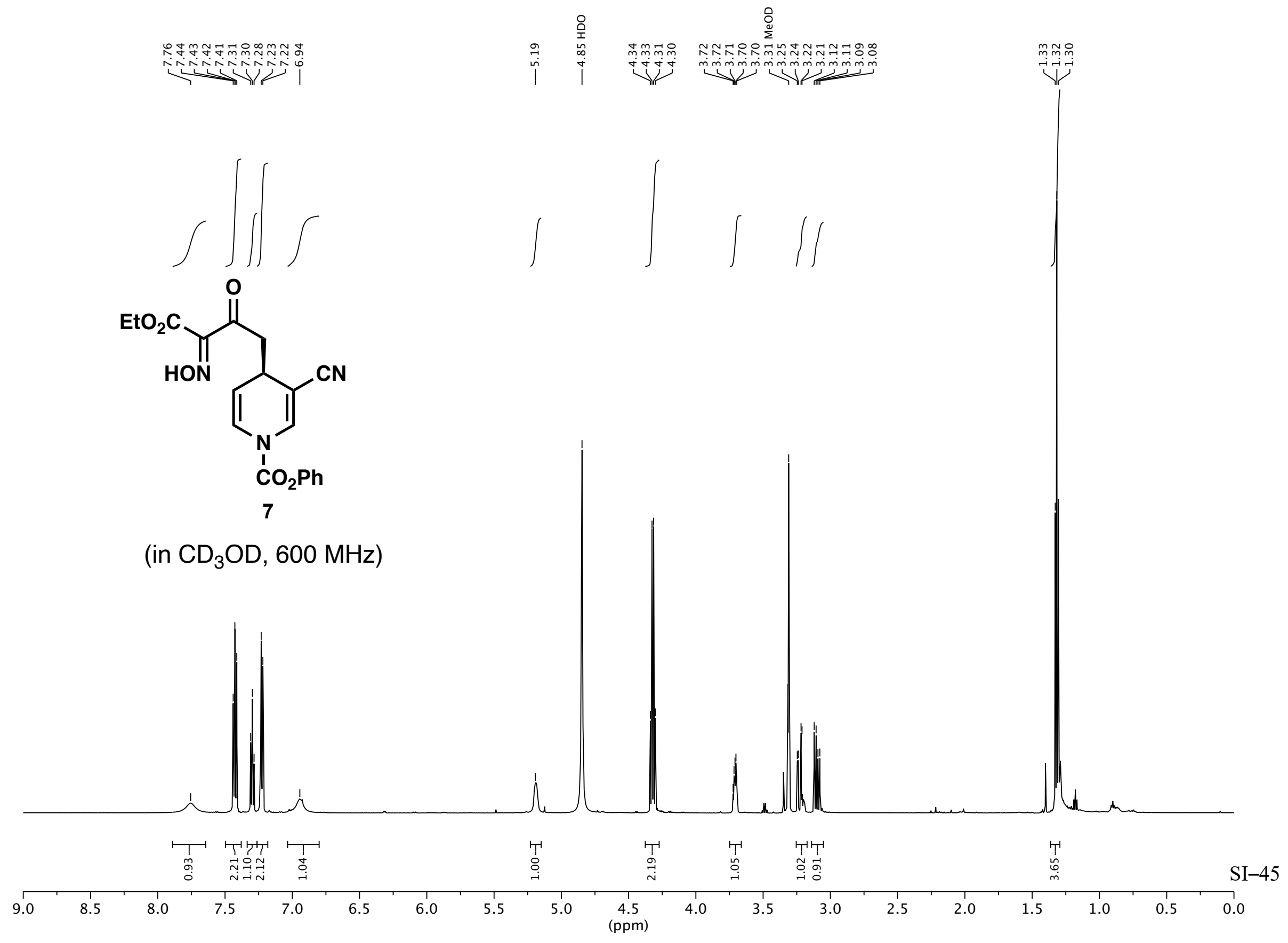



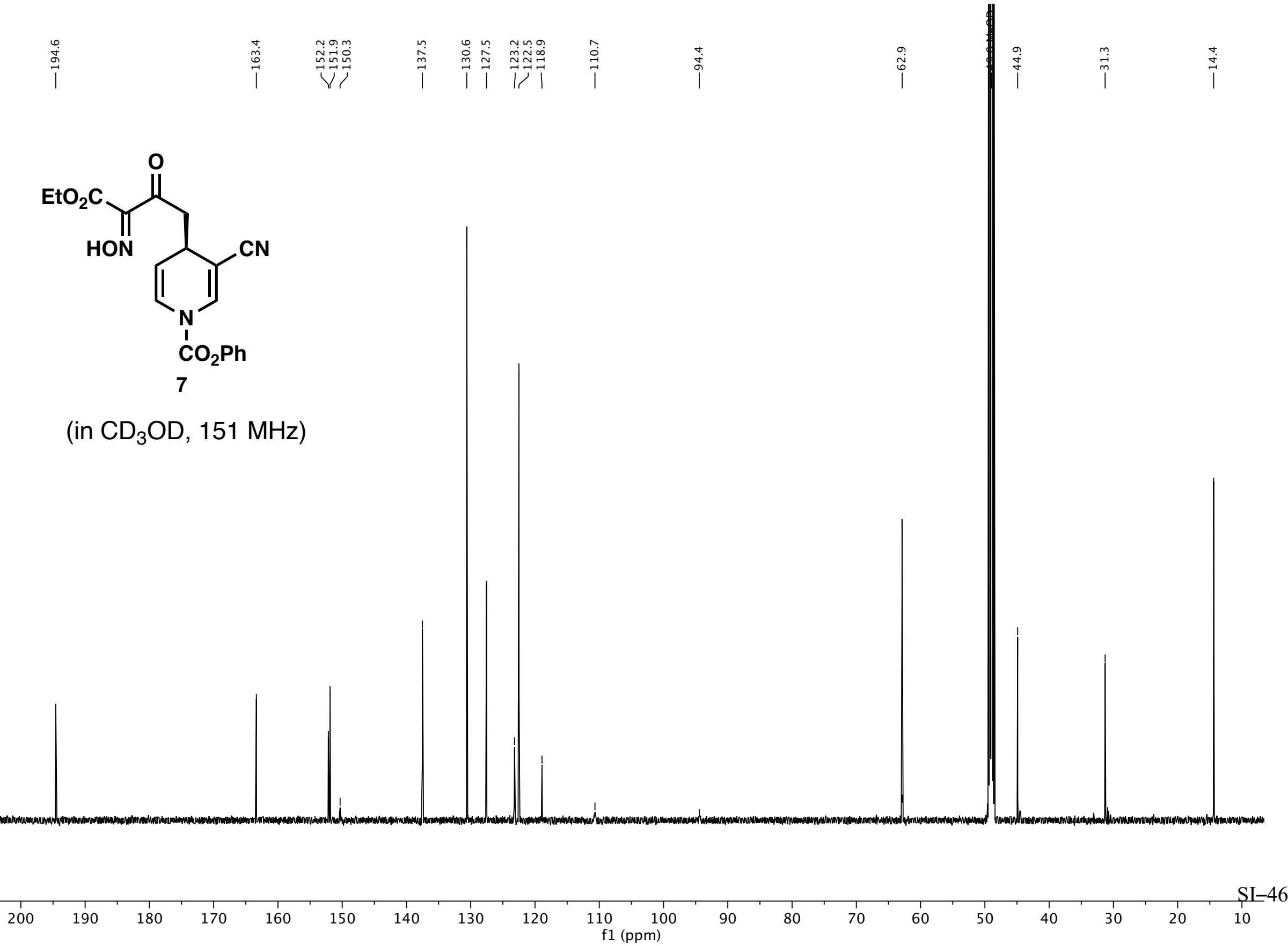


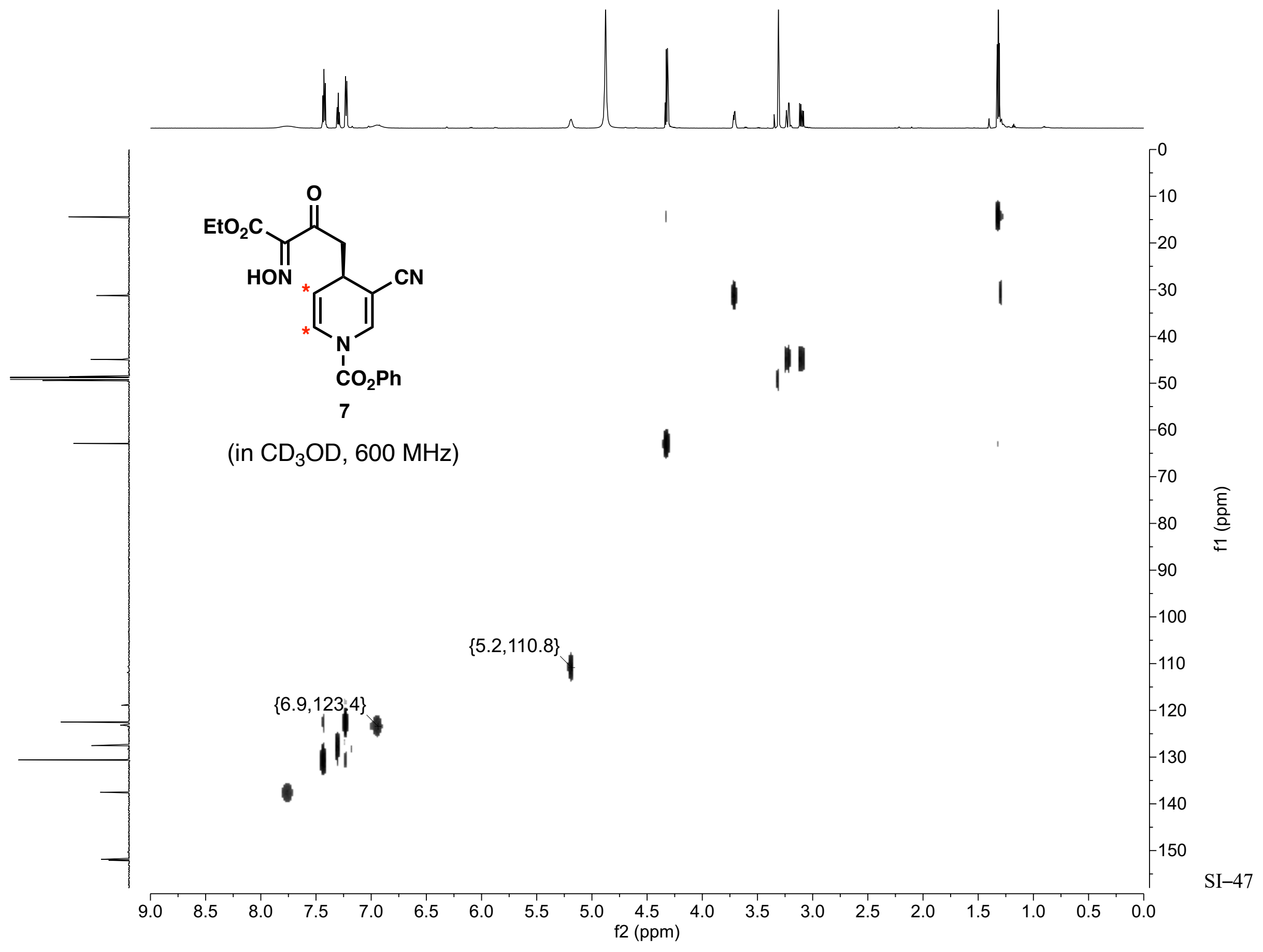




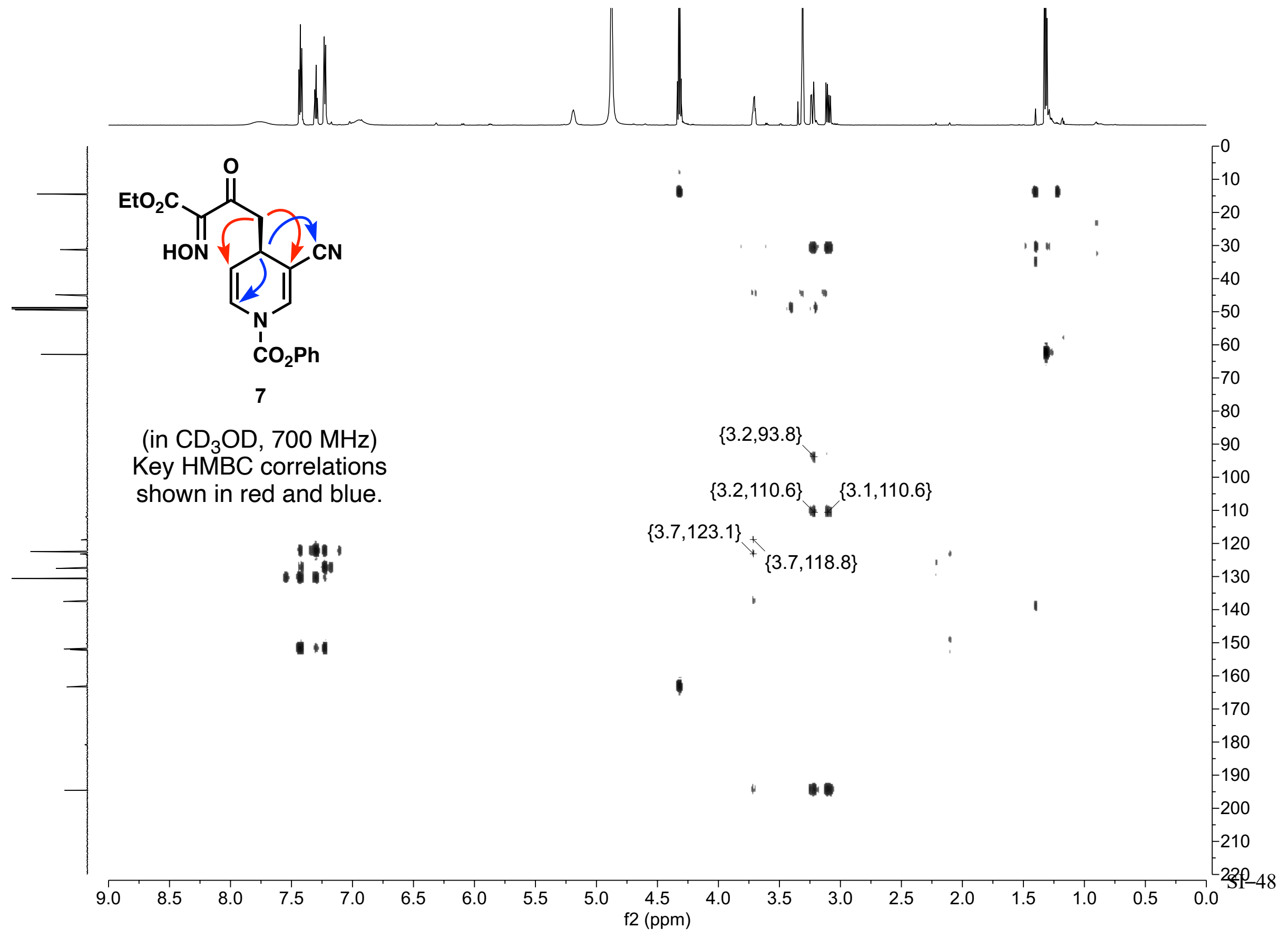




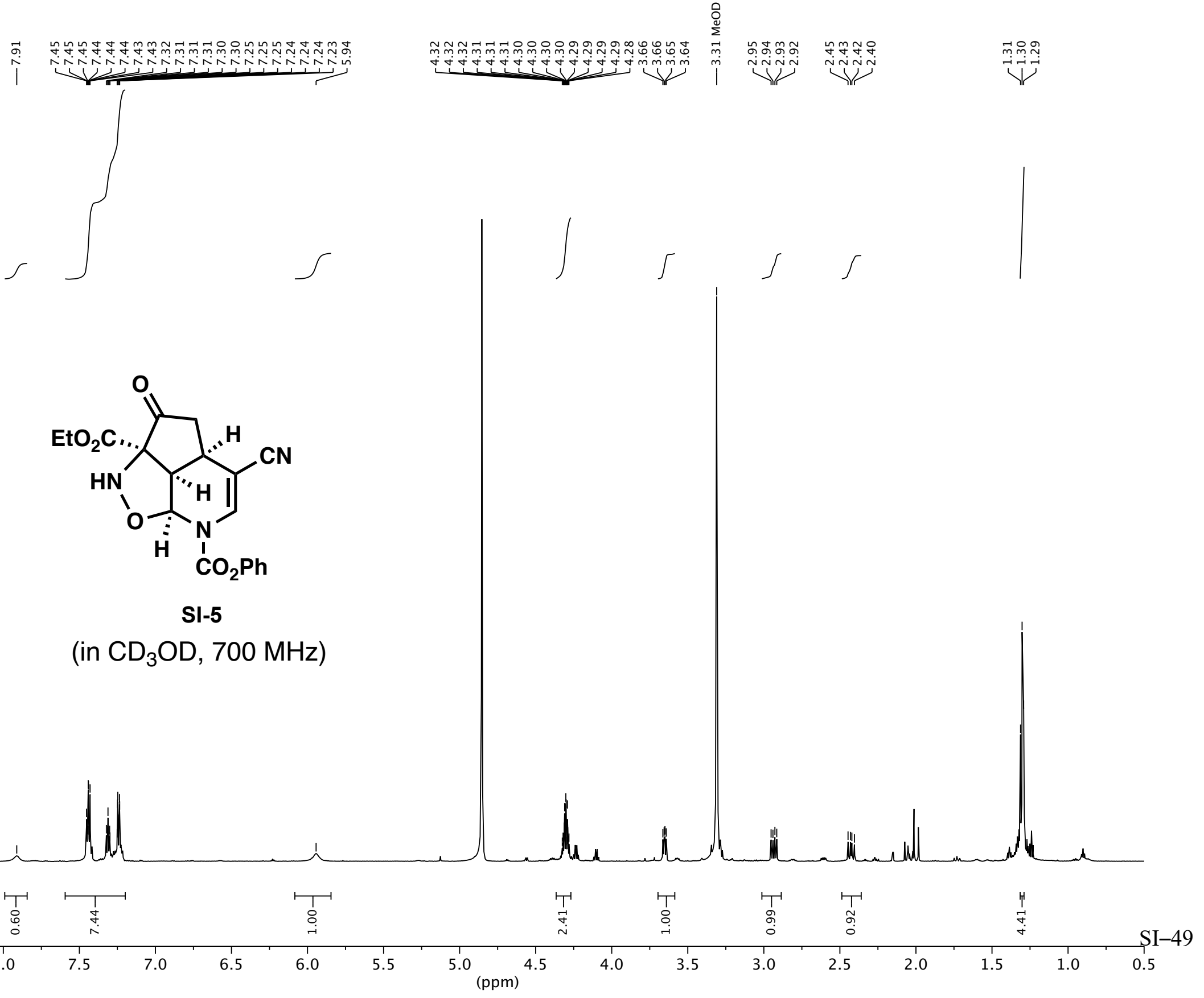




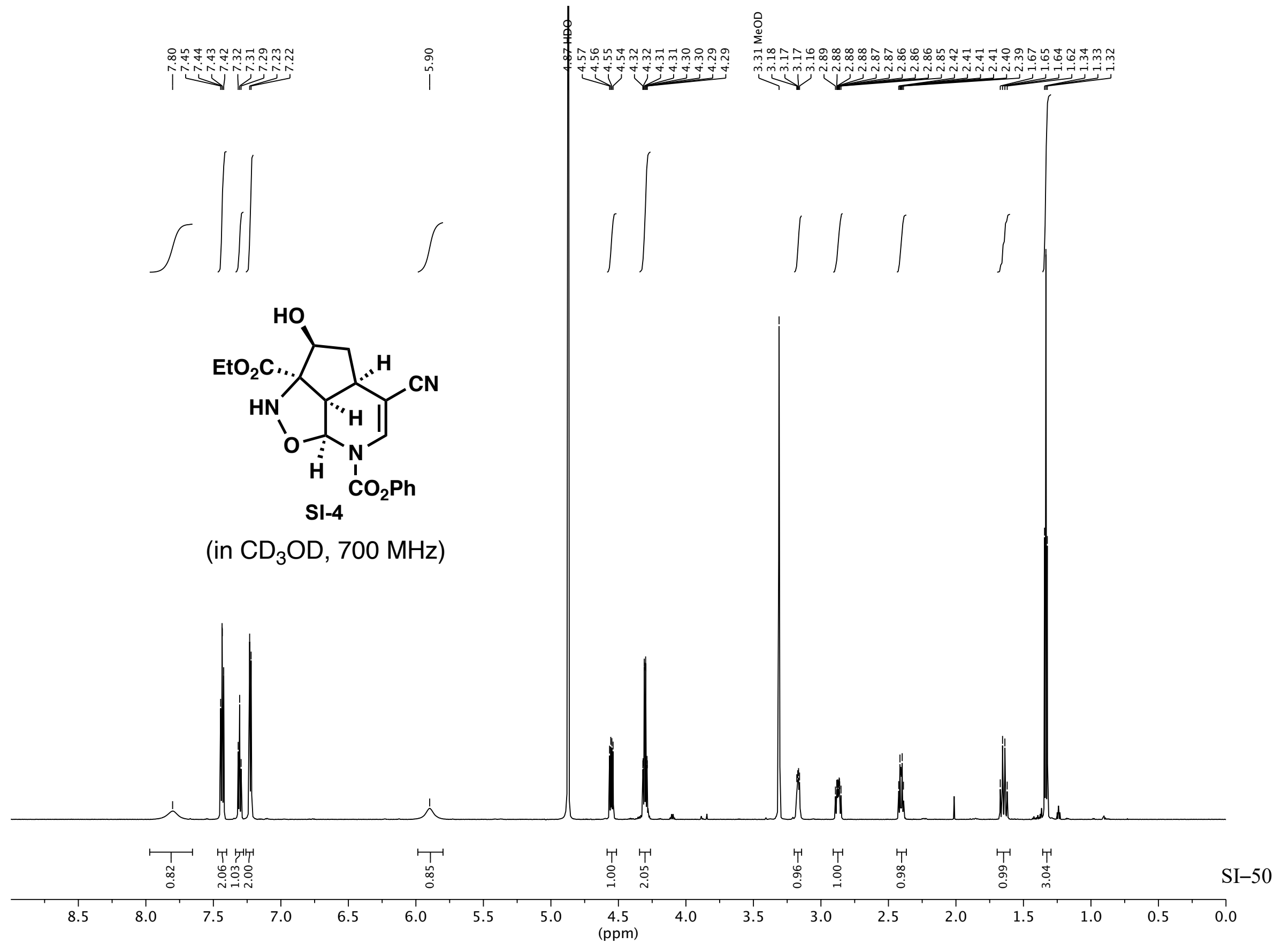



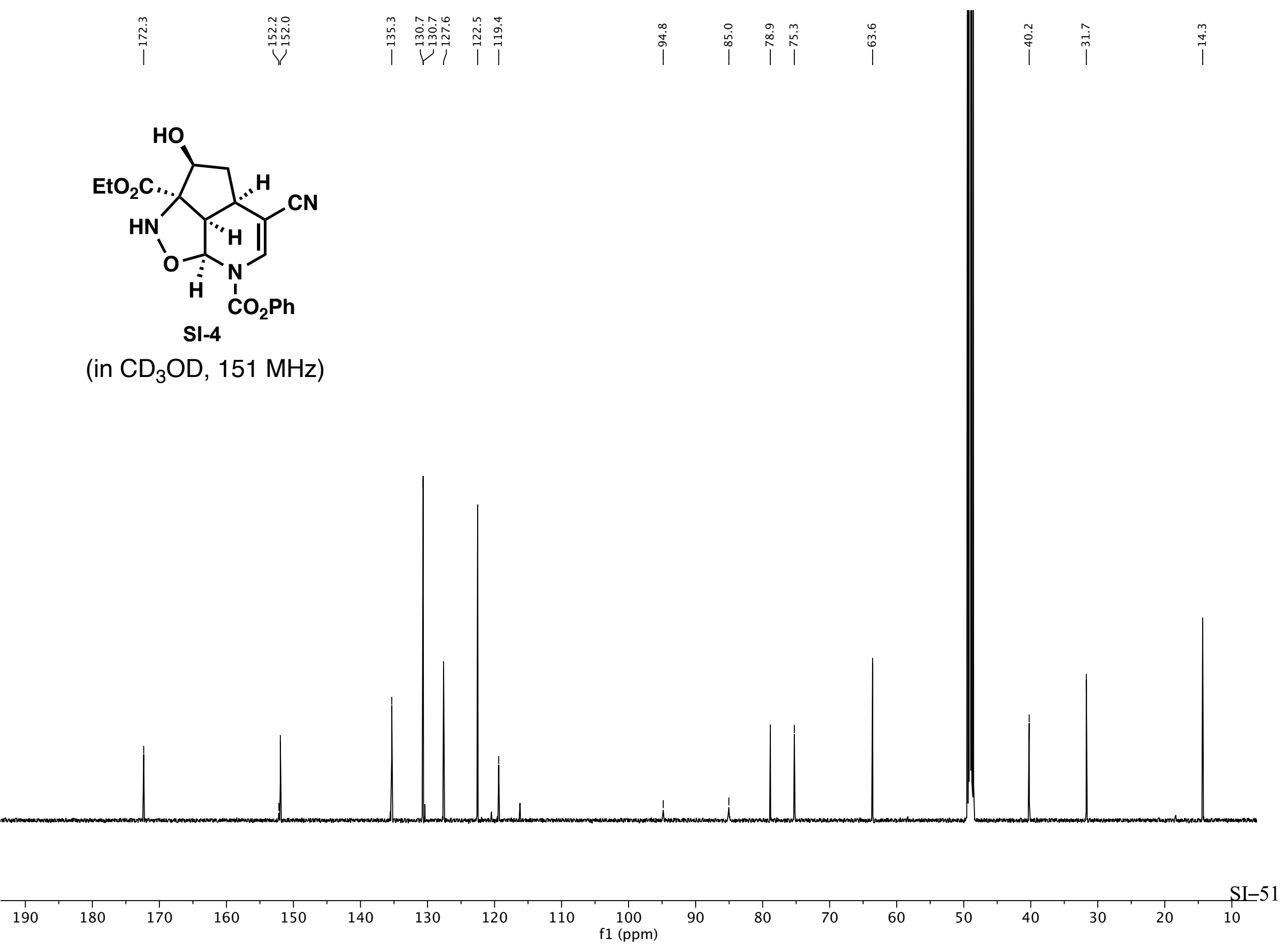


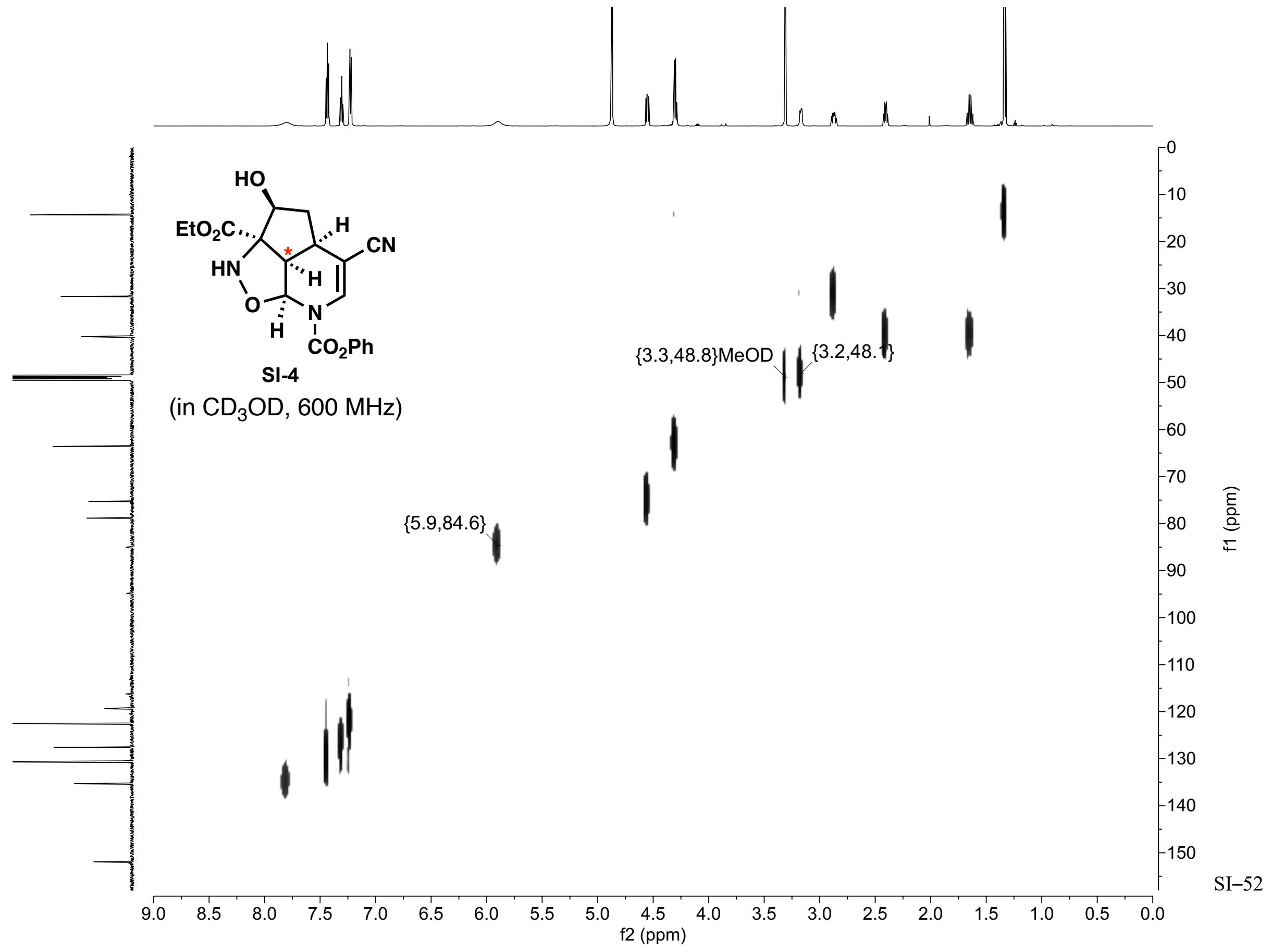




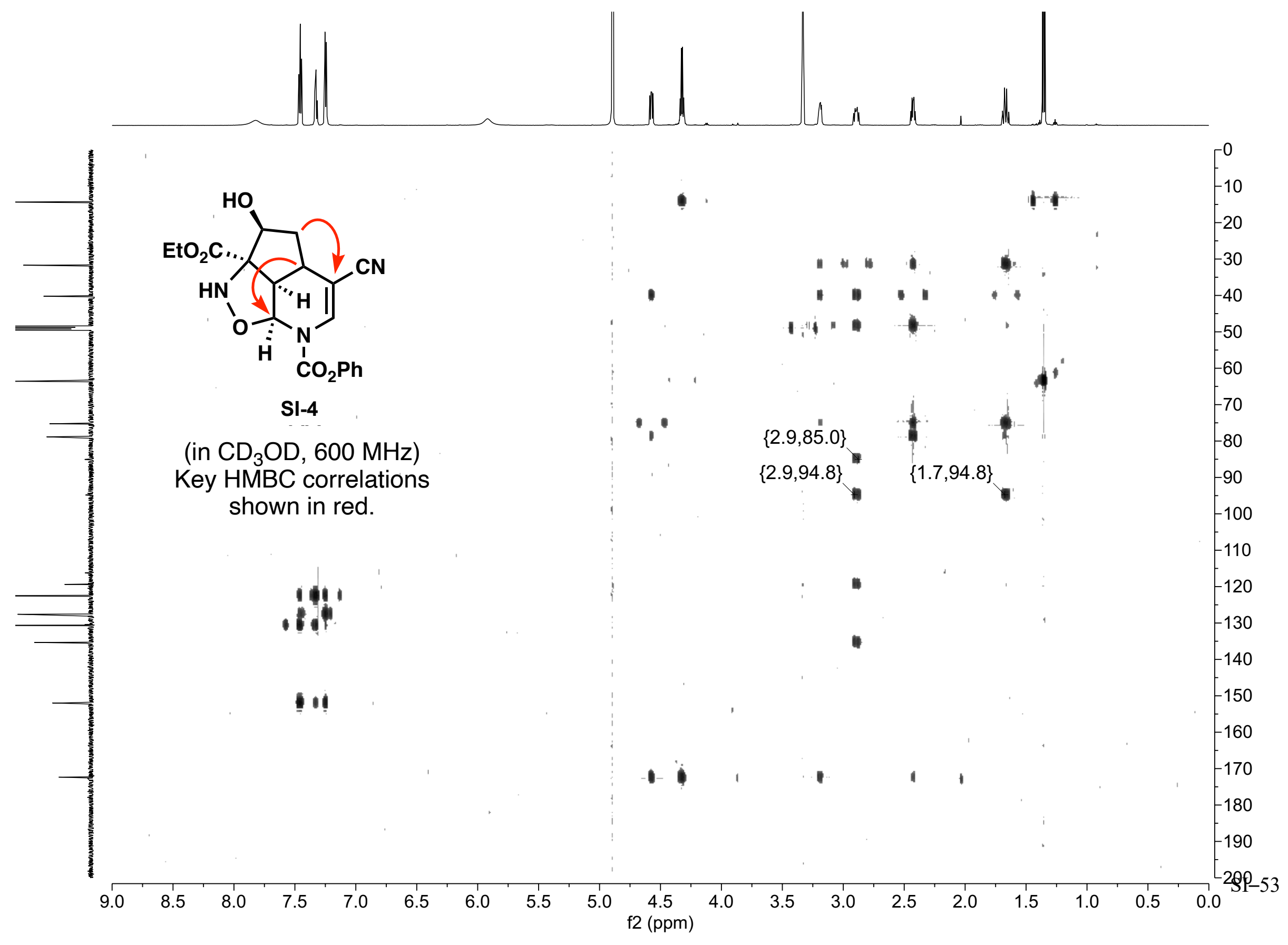




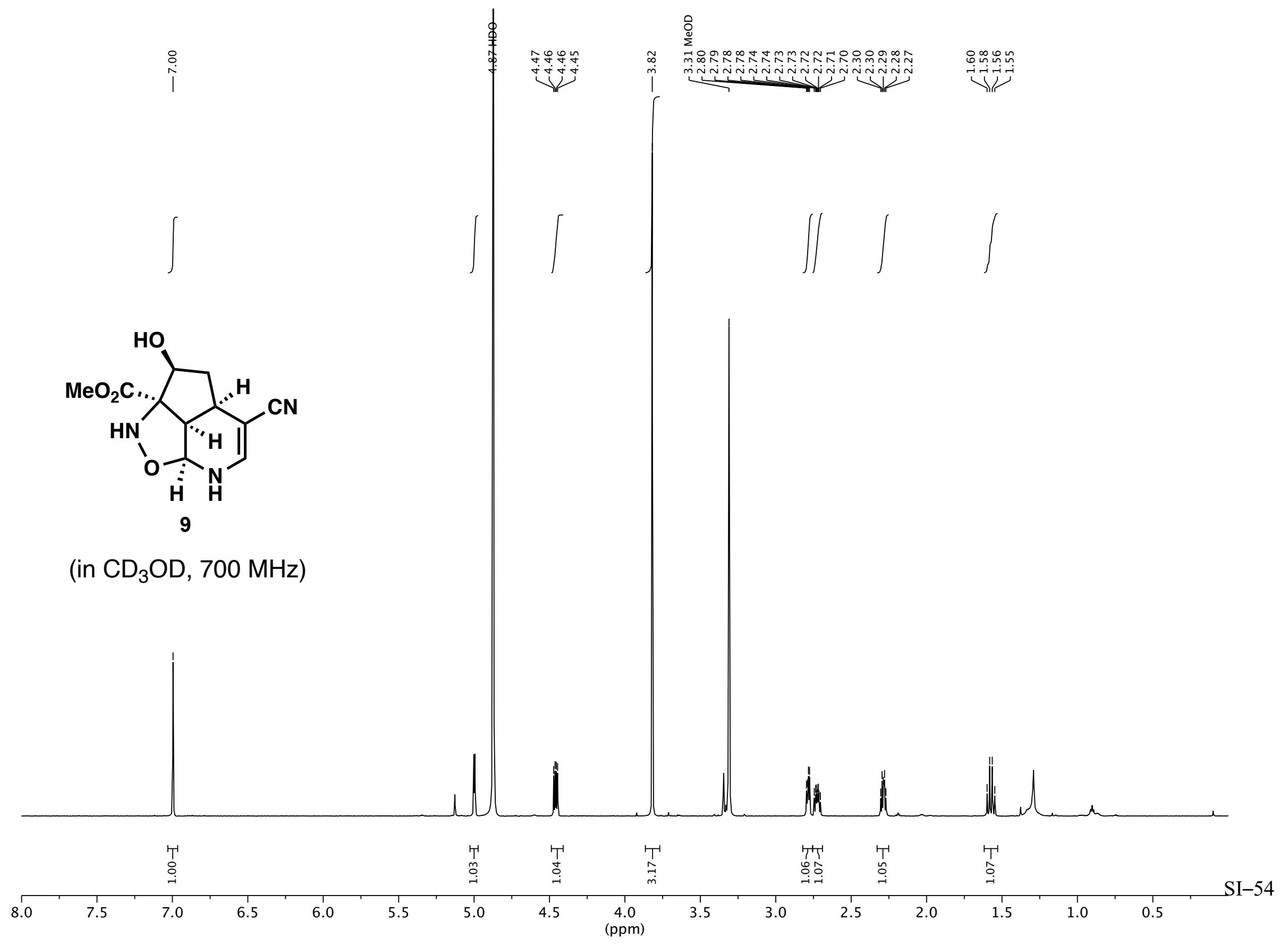



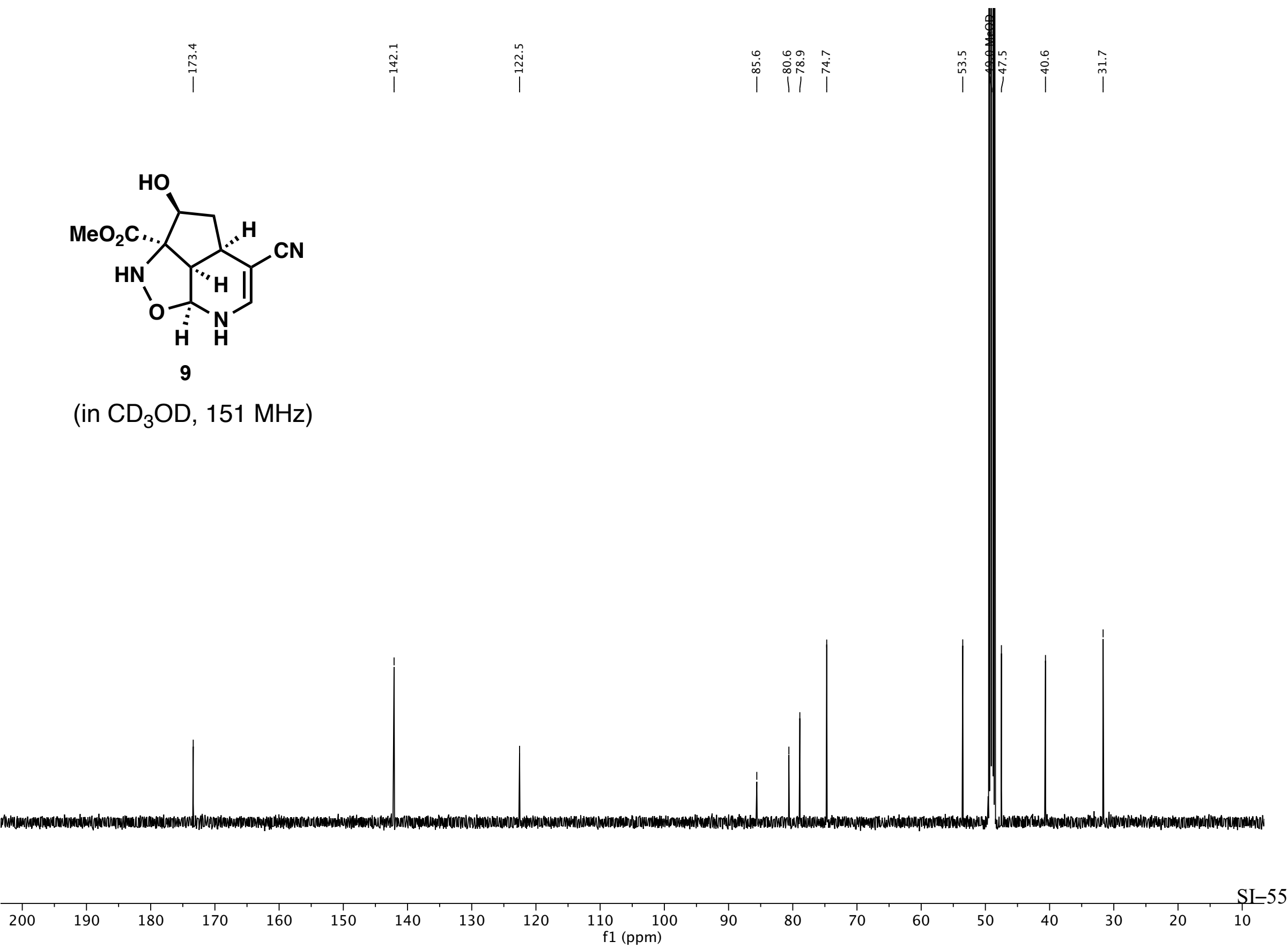


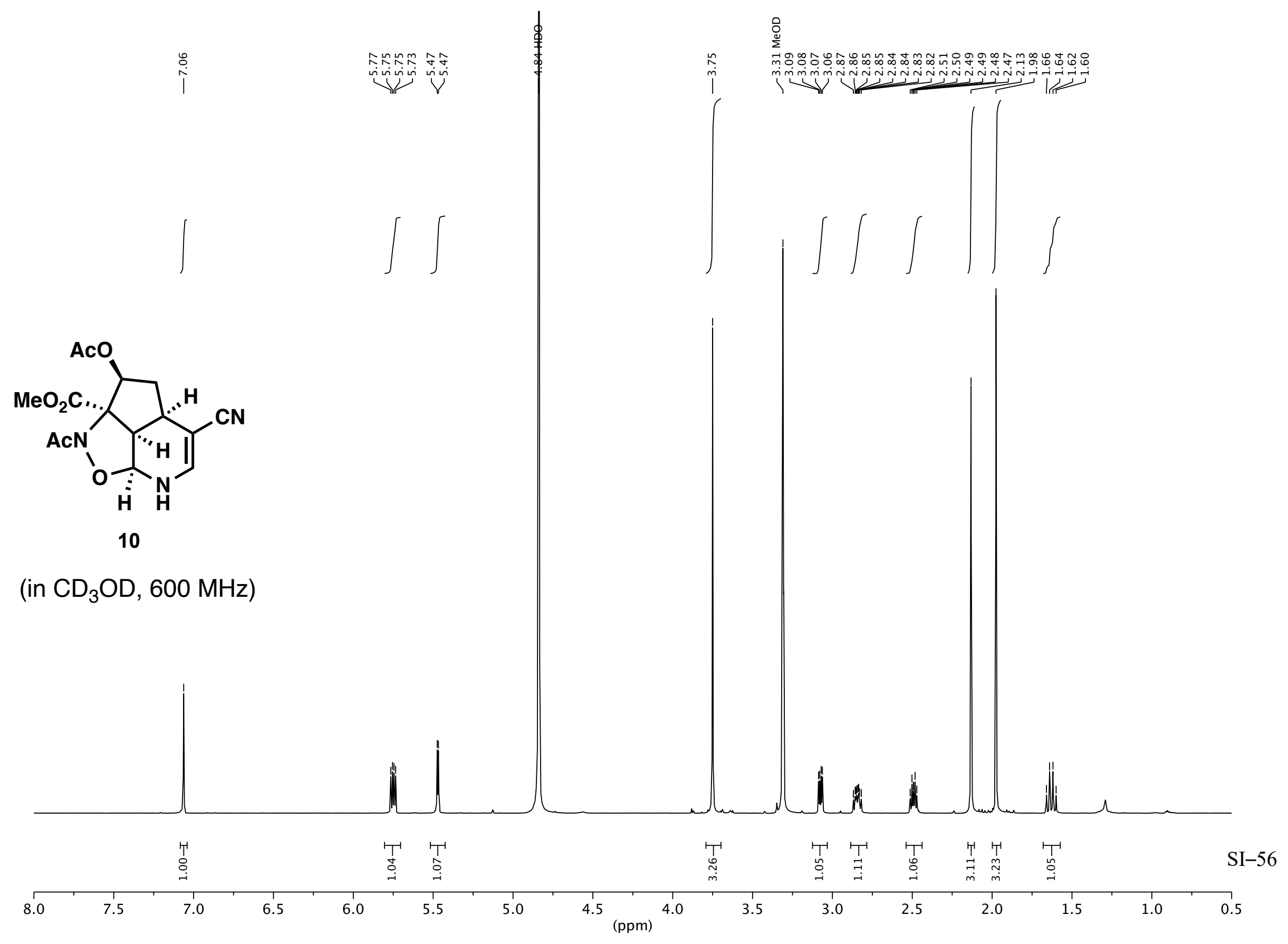




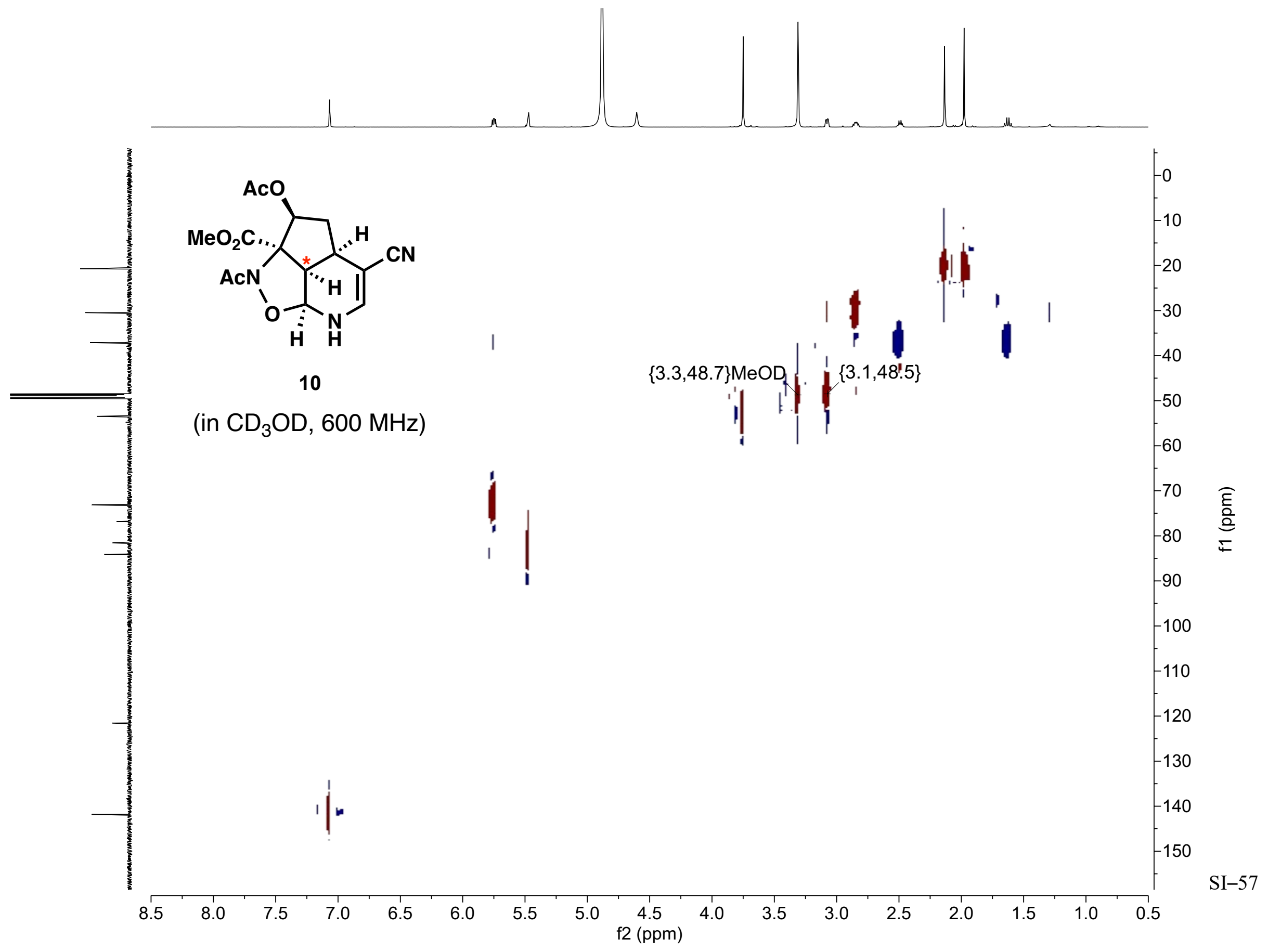



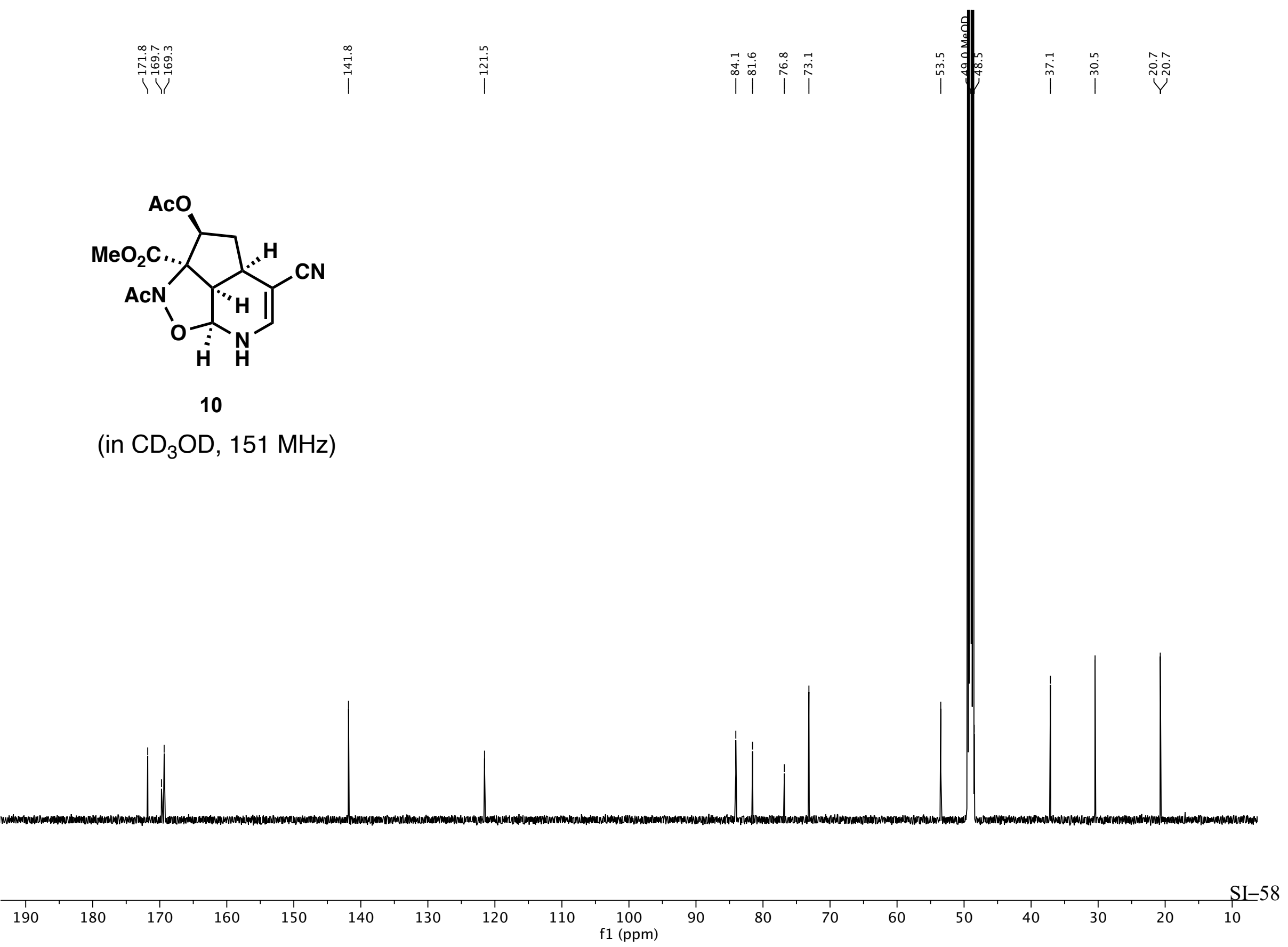


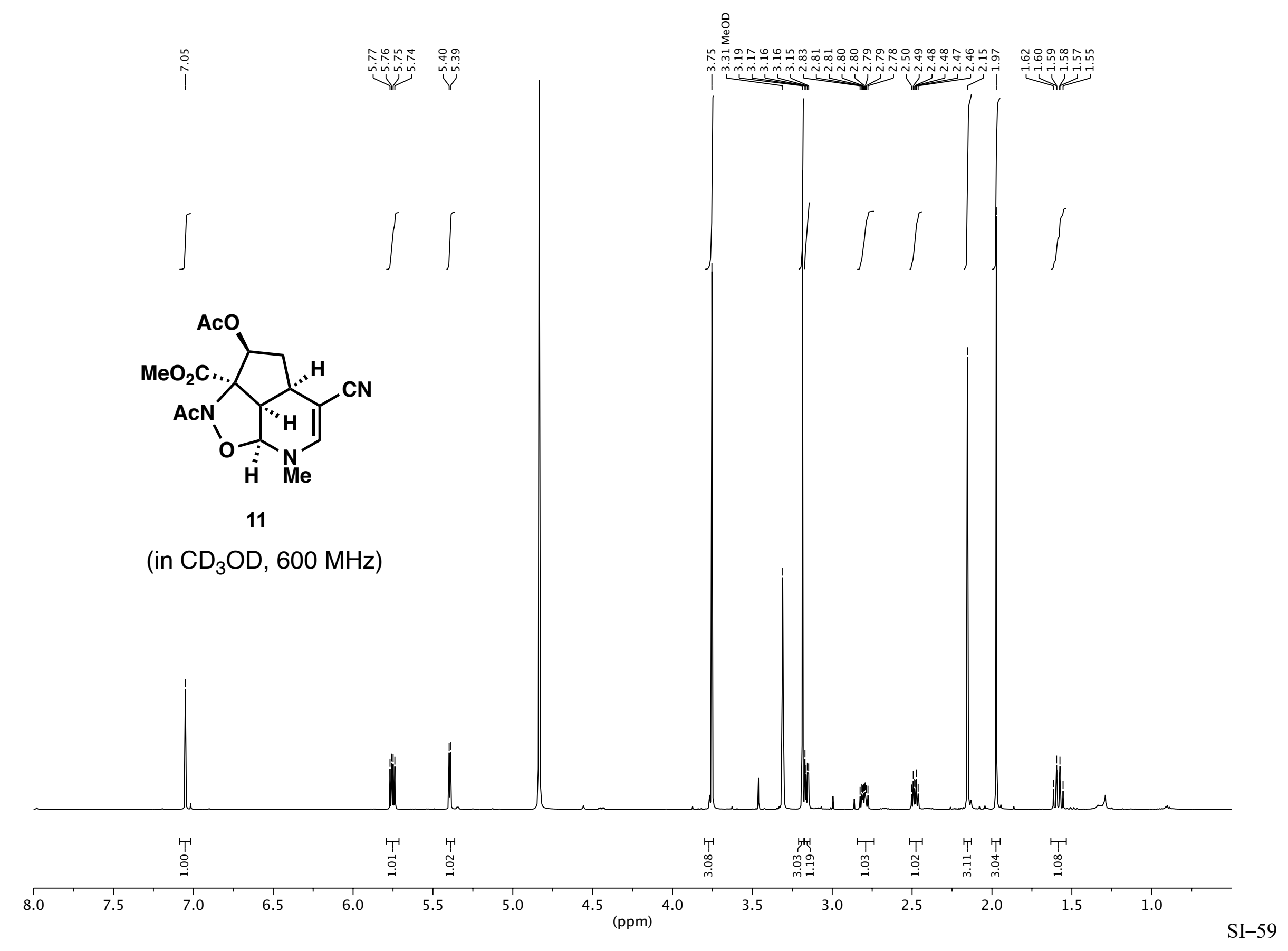




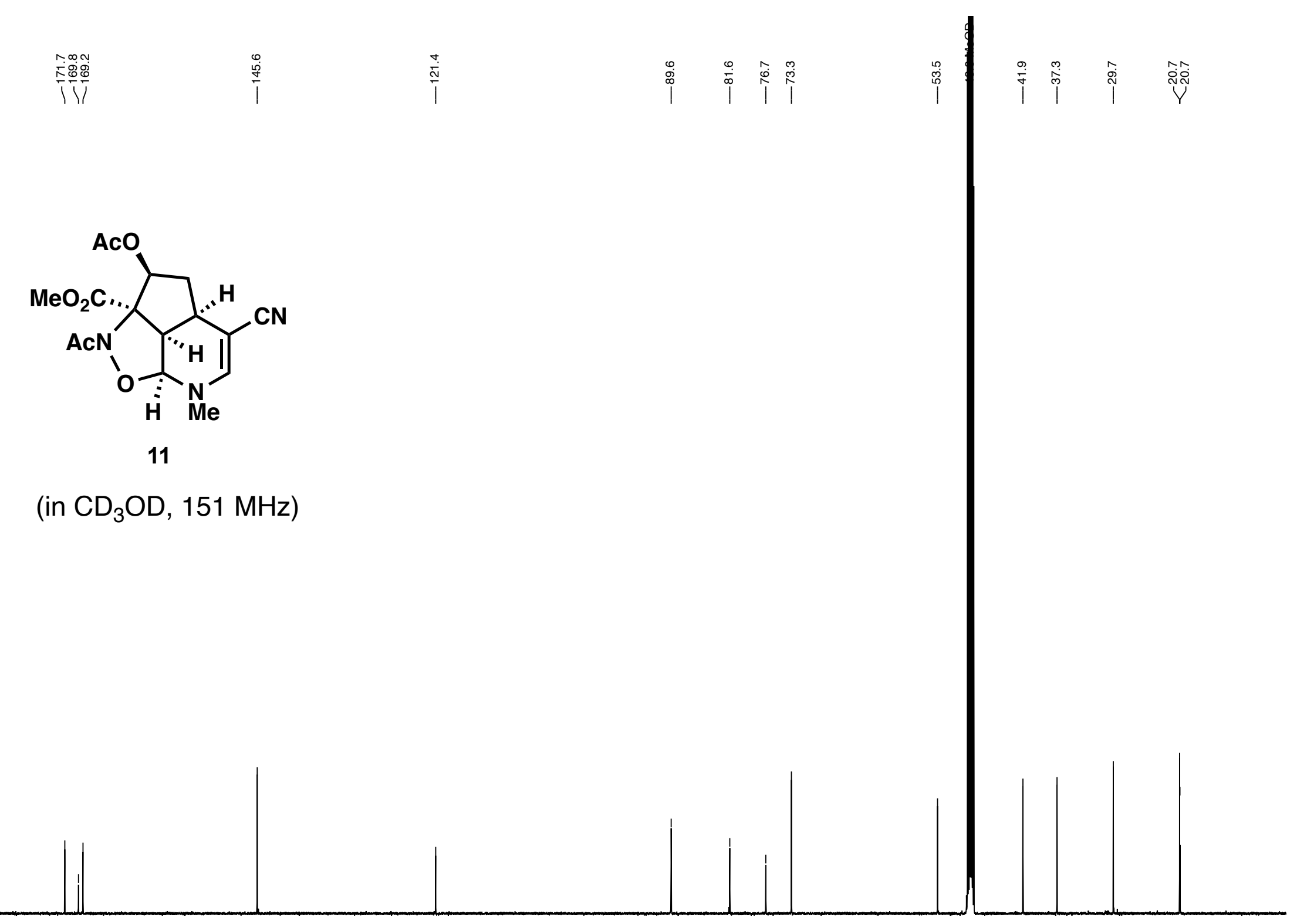

SI-60 


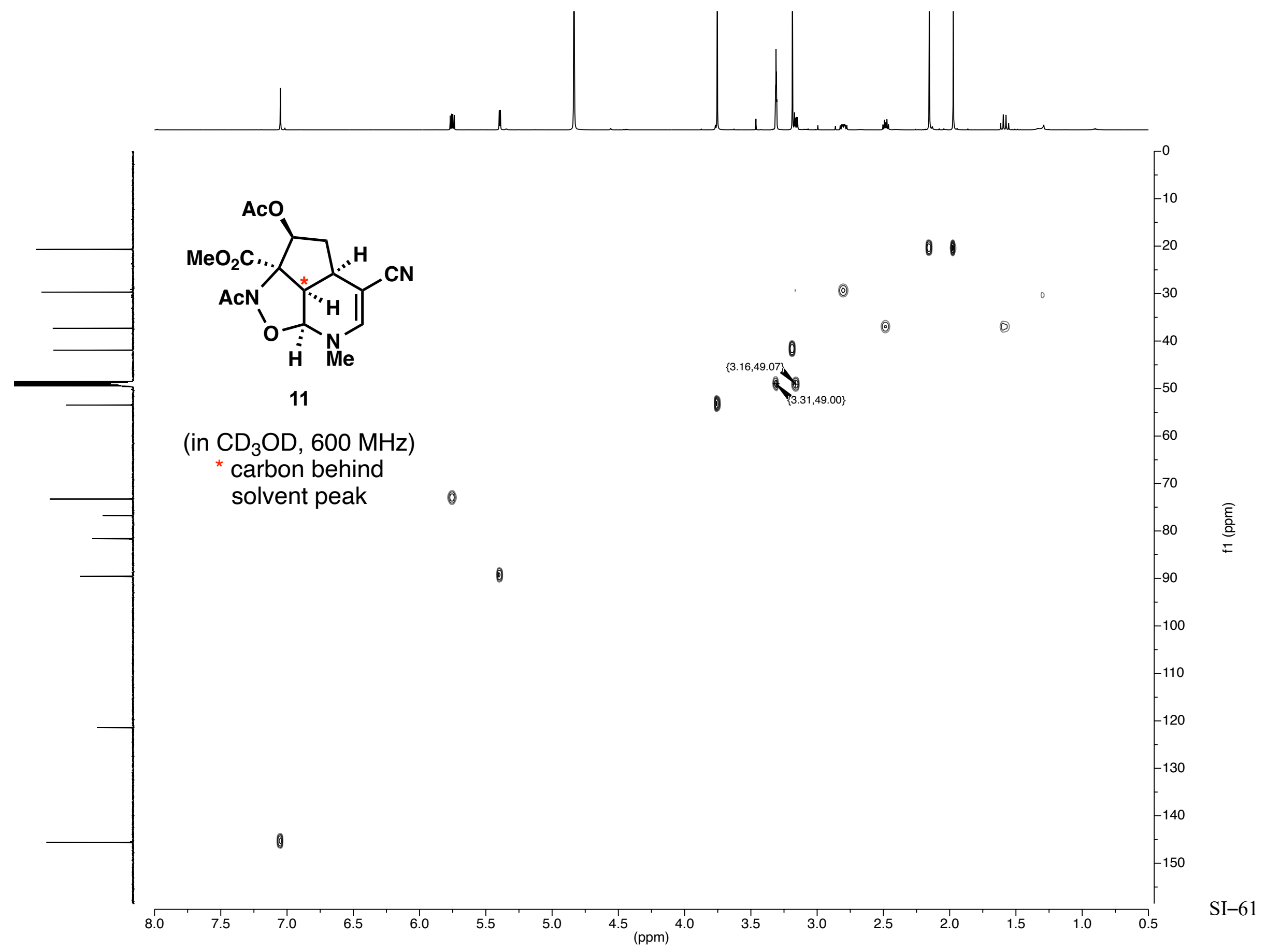




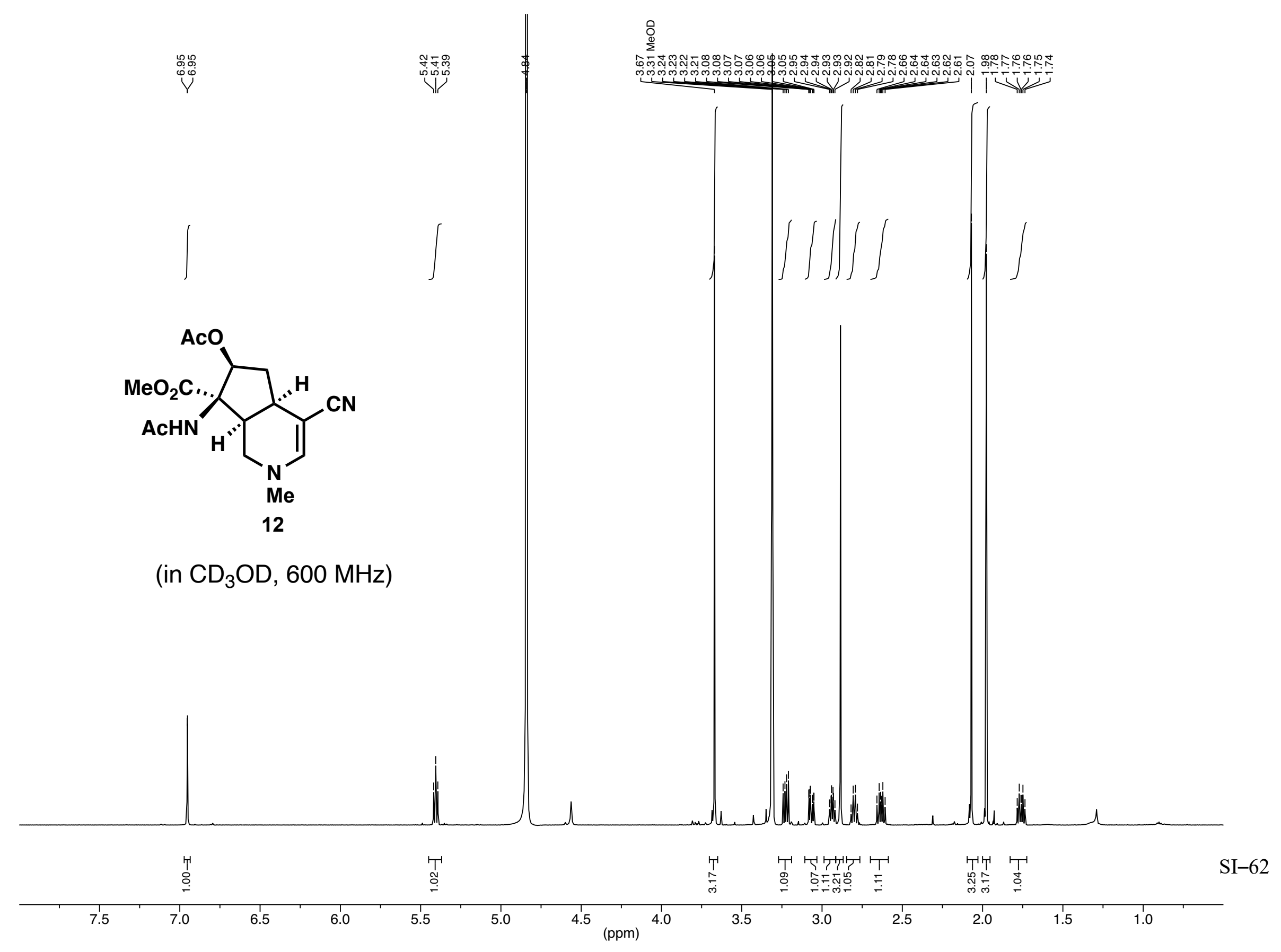




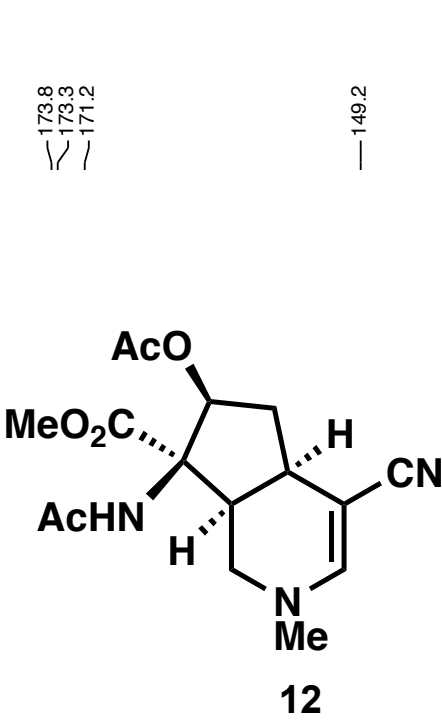

(in $\mathrm{CD}_{3} \mathrm{OD}, 151 \mathrm{MHz}$ )

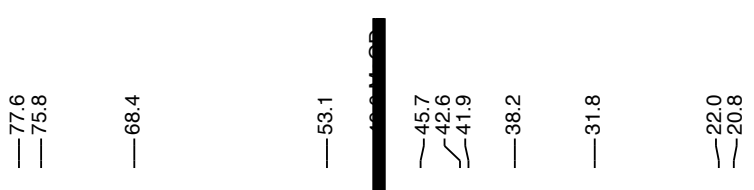

SI-63 

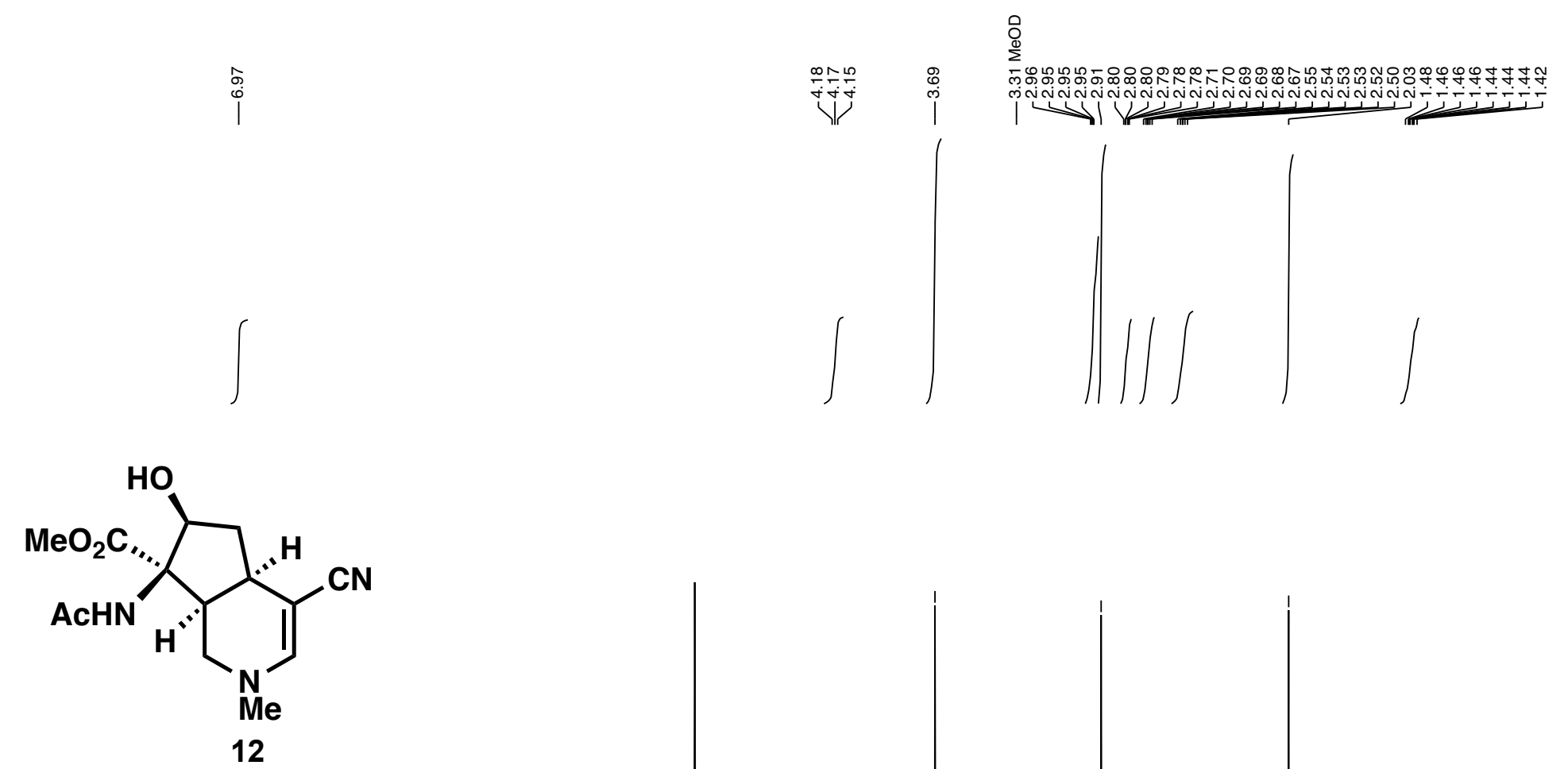

(in $\mathrm{CD}_{3} \mathrm{OD}, 151 \mathrm{MHz}$ )

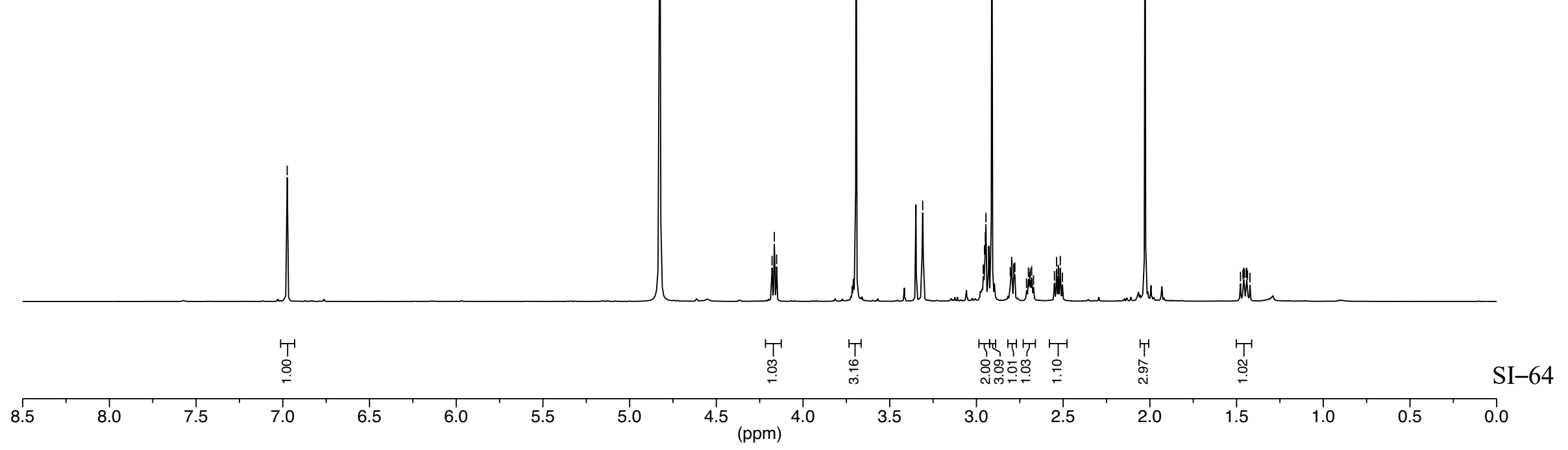




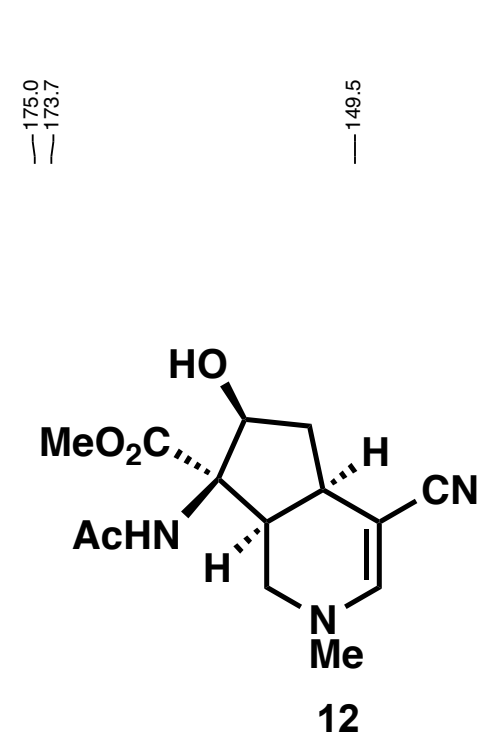

(in $\mathrm{CD}_{3} \mathrm{OD}, 600 \mathrm{MHz}$ )

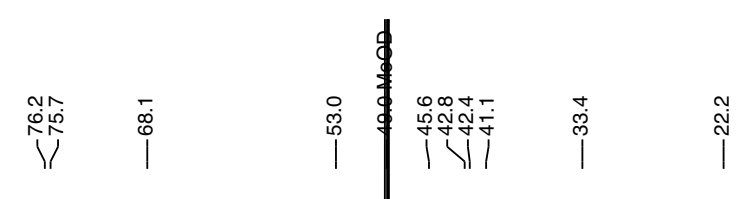




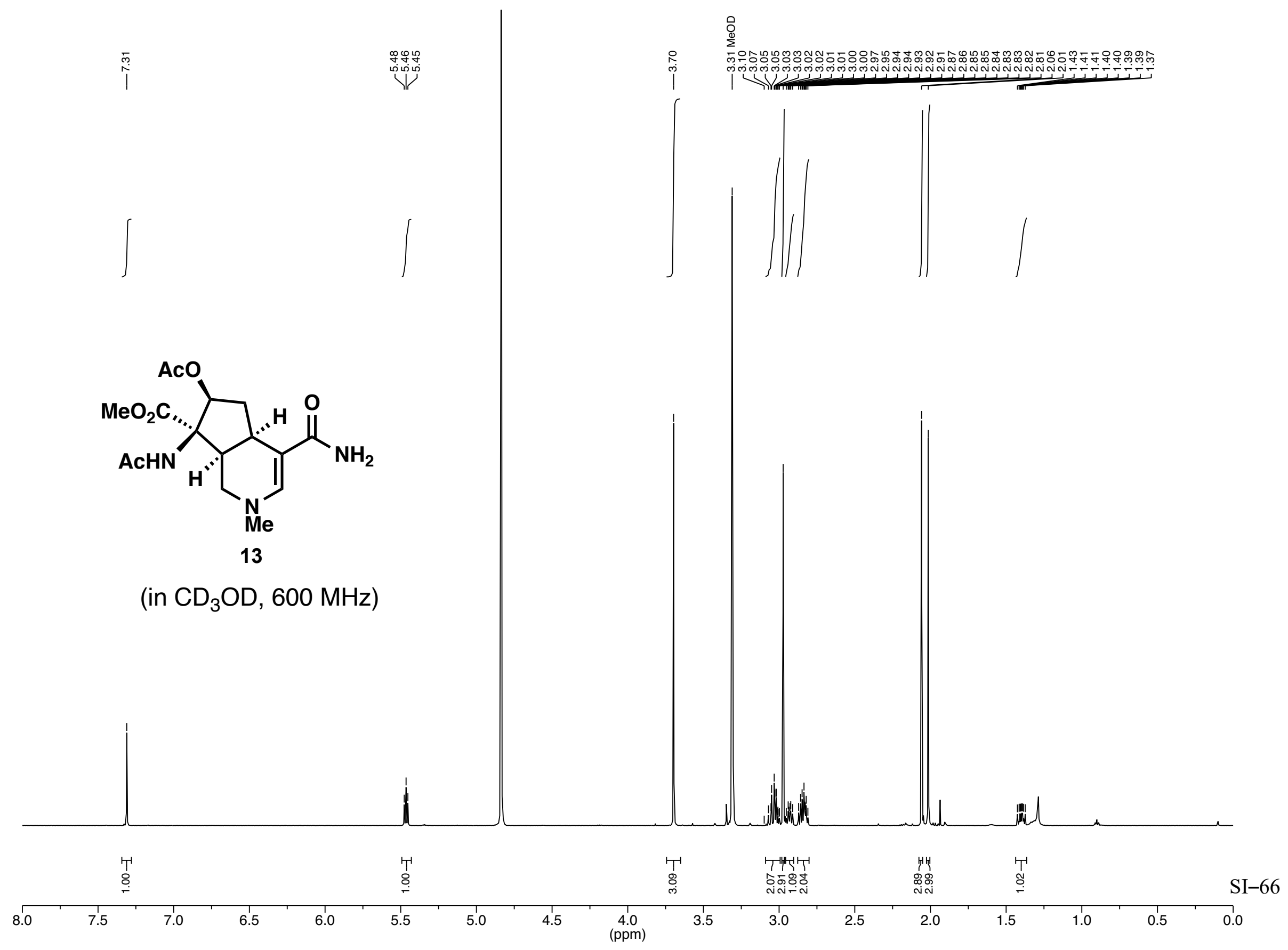




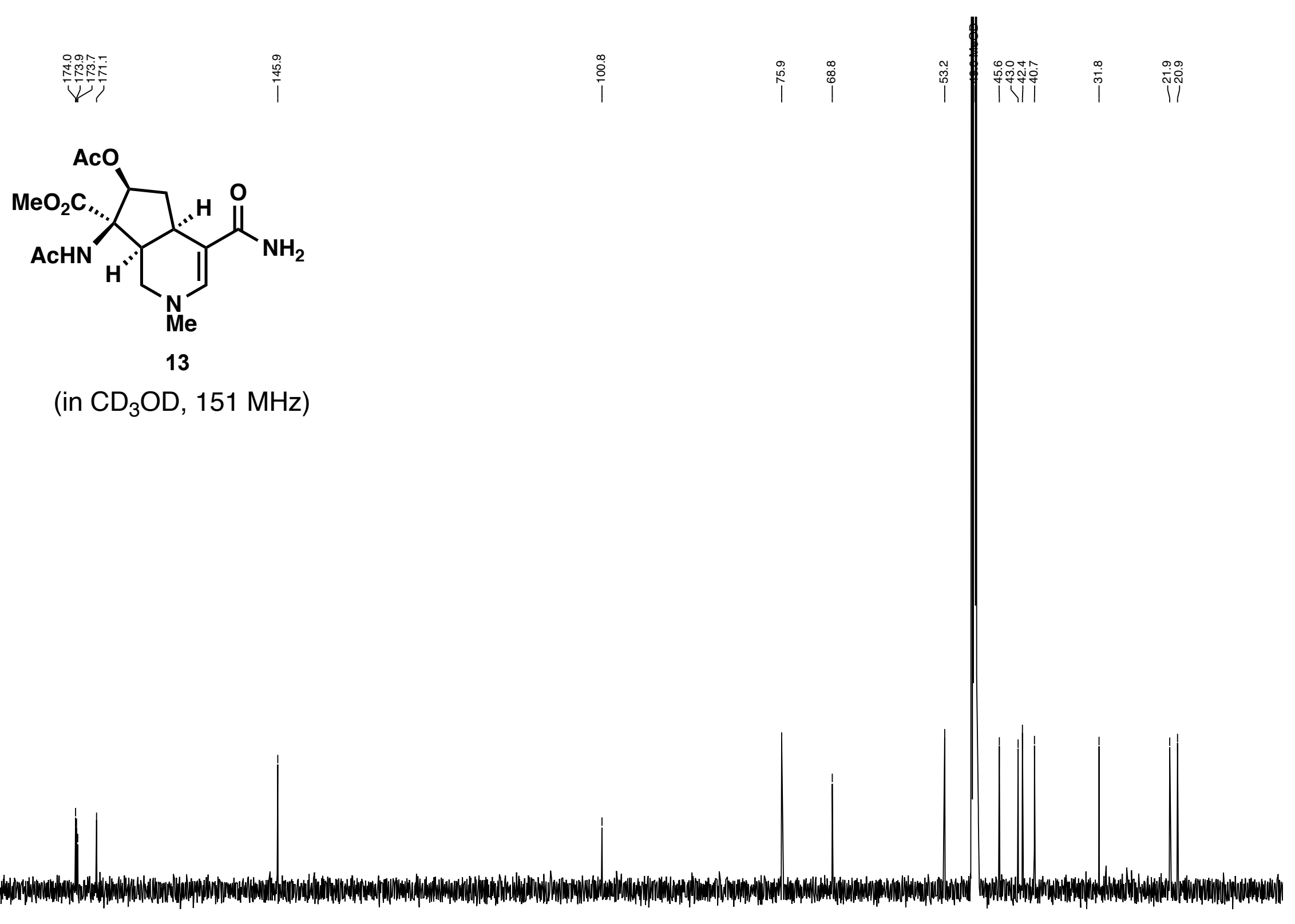

SI-67 


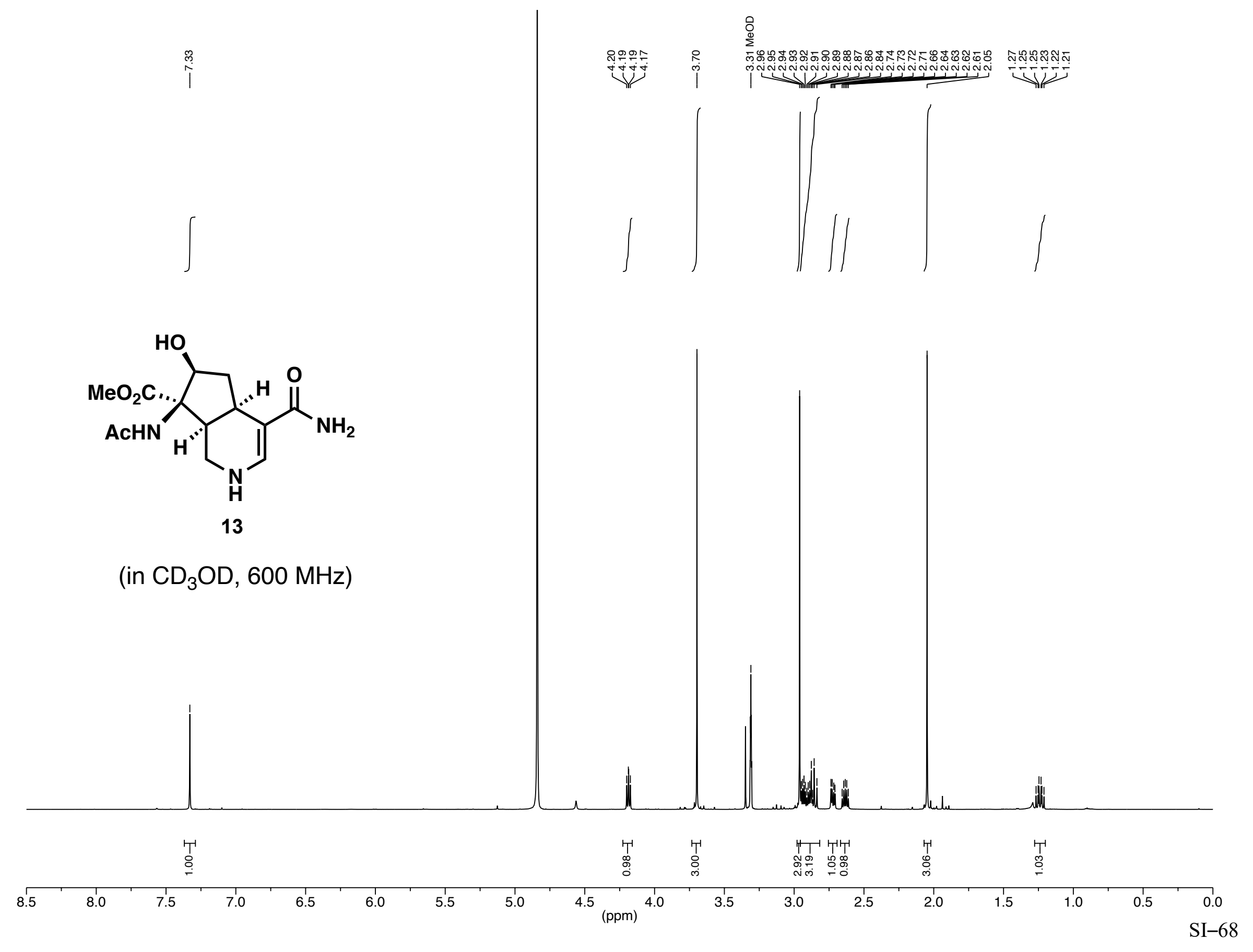



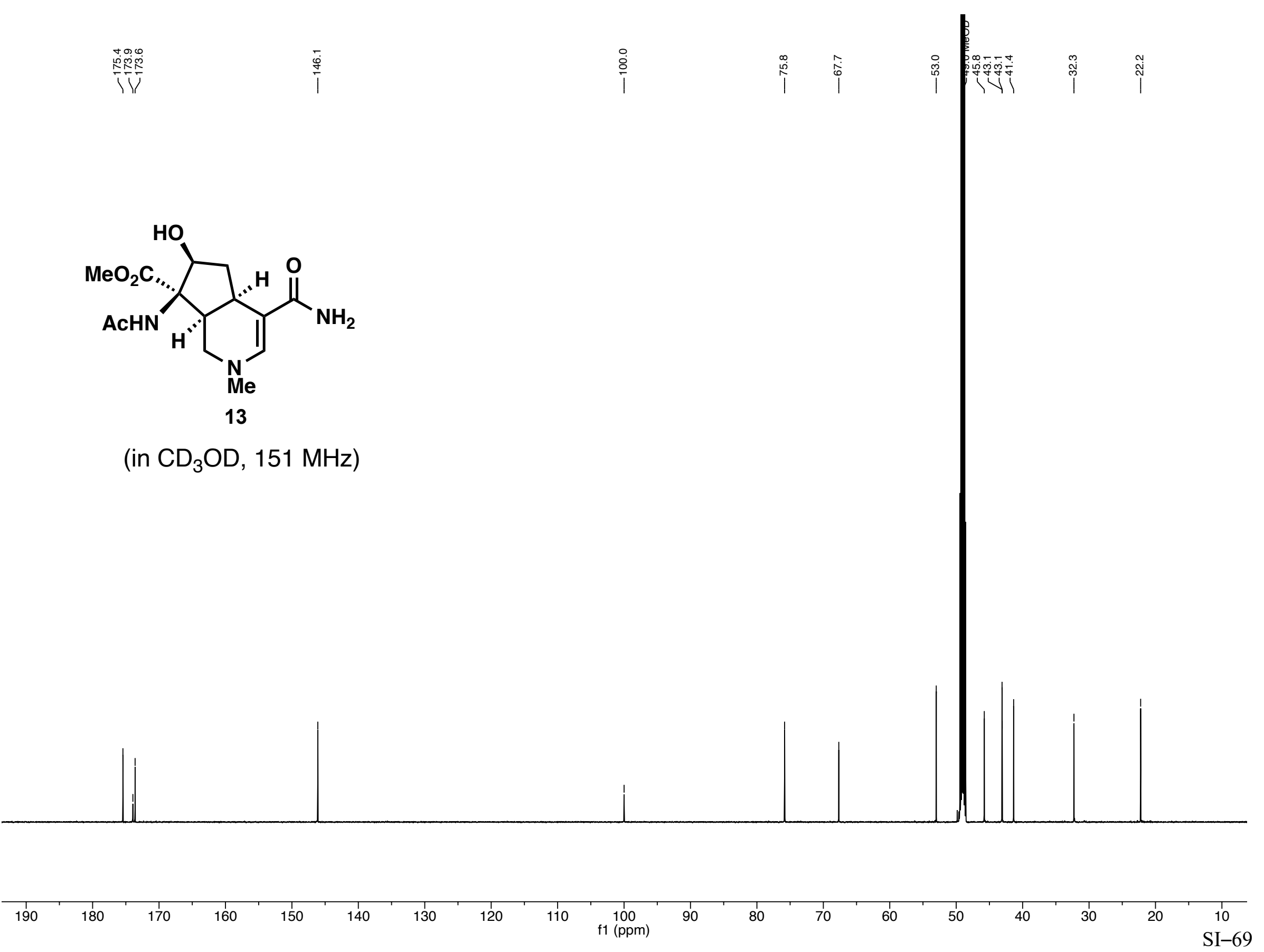


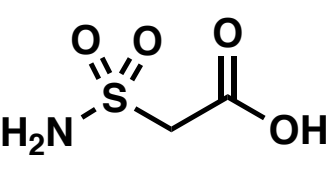

15

(in $\mathrm{CD}_{3} \mathrm{OD}, 600 \mathrm{MHz}$ )
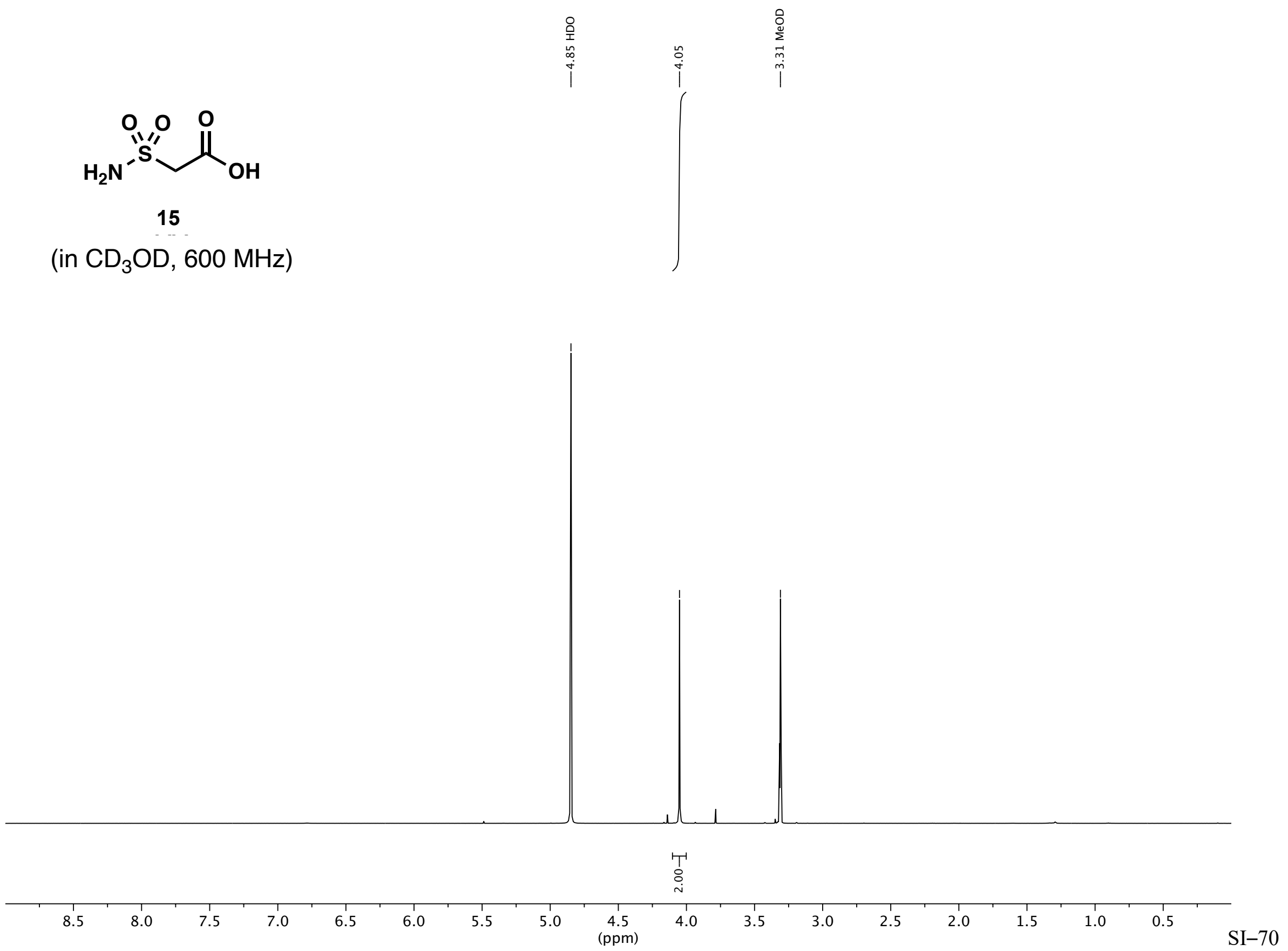
$\stackrel{0}{\stackrel{0}{1}}$

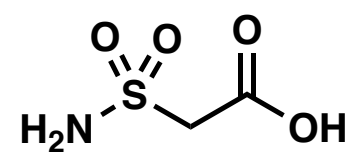

15

(in $\mathrm{CD}_{3} \mathrm{OD}, 151 \mathrm{MHz}$ ) 


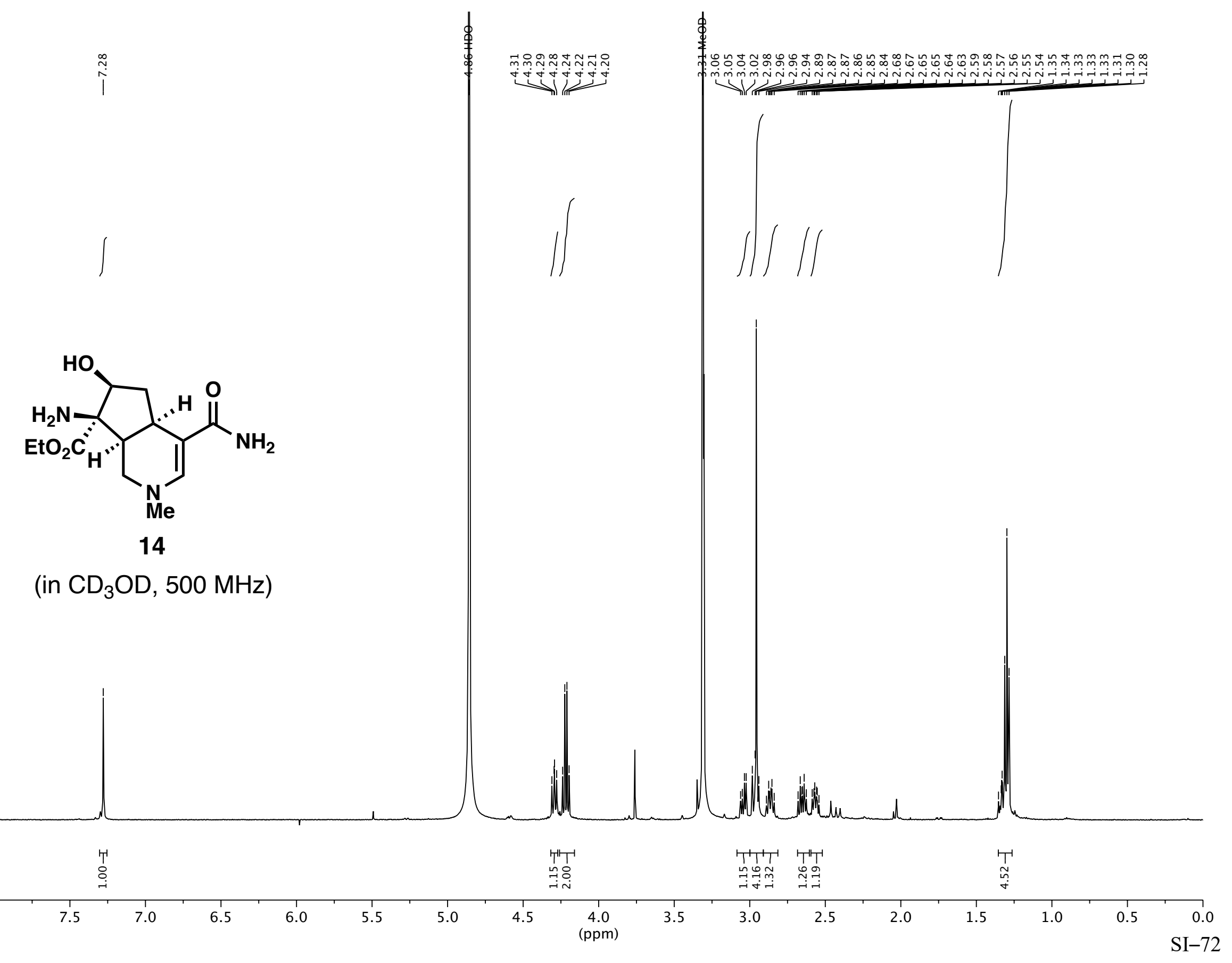




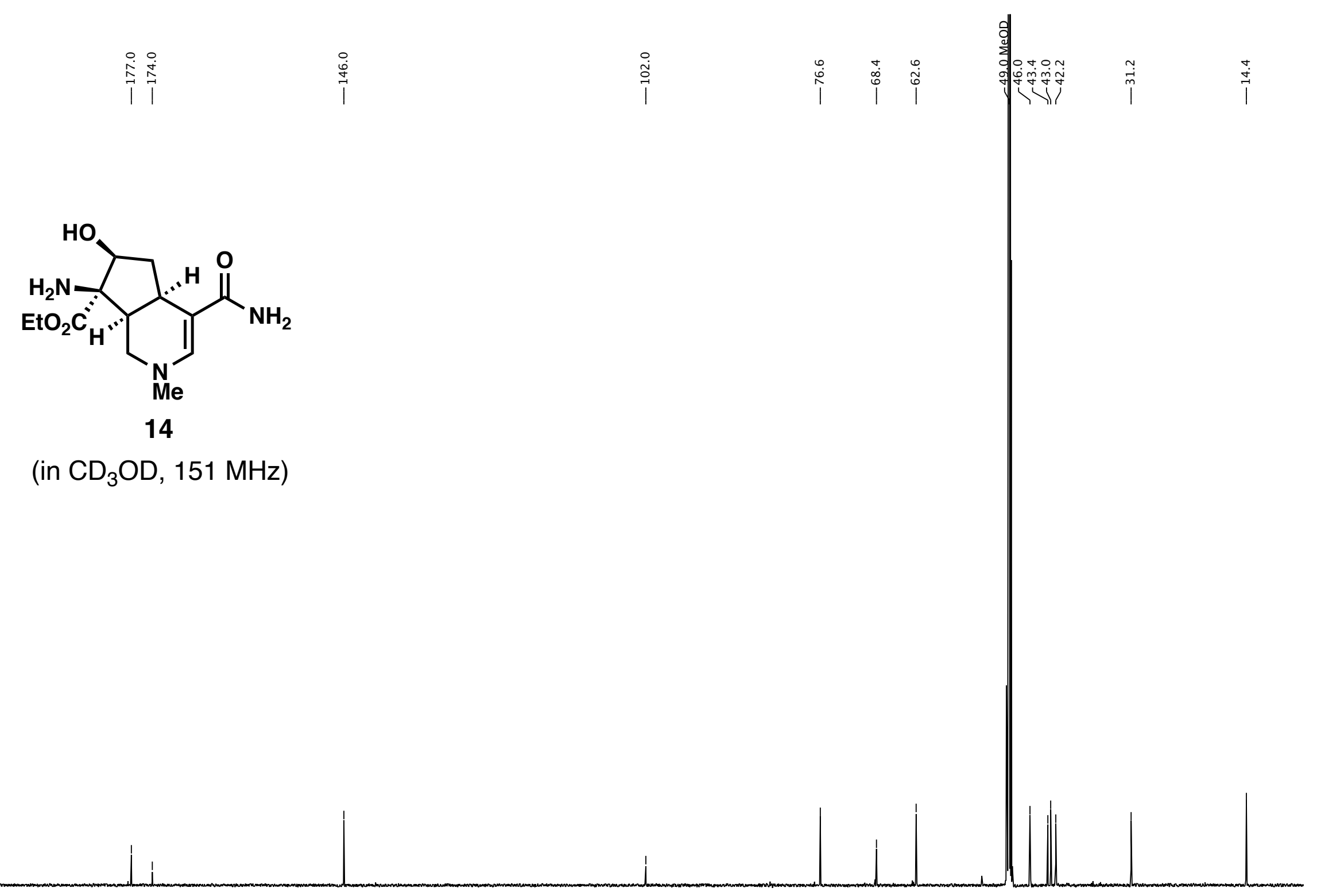

\begin{tabular}{|c|c|c|c|c|c|c|c|c|c|c|c|c|c|c|c|c|c|}
\hline 200 & 190 & $\begin{array}{c}1 \\
180\end{array}$ & 170 & 160 & 150 & 140 & 130 & 120 & $\begin{array}{c}110 \quad 100 \\
\mathrm{f} 1(\mathrm{ppm})\end{array}$ & 90 & 80 & 70 & 60 & 50 & 40 & 30 & 20 \\
\hline
\end{tabular}




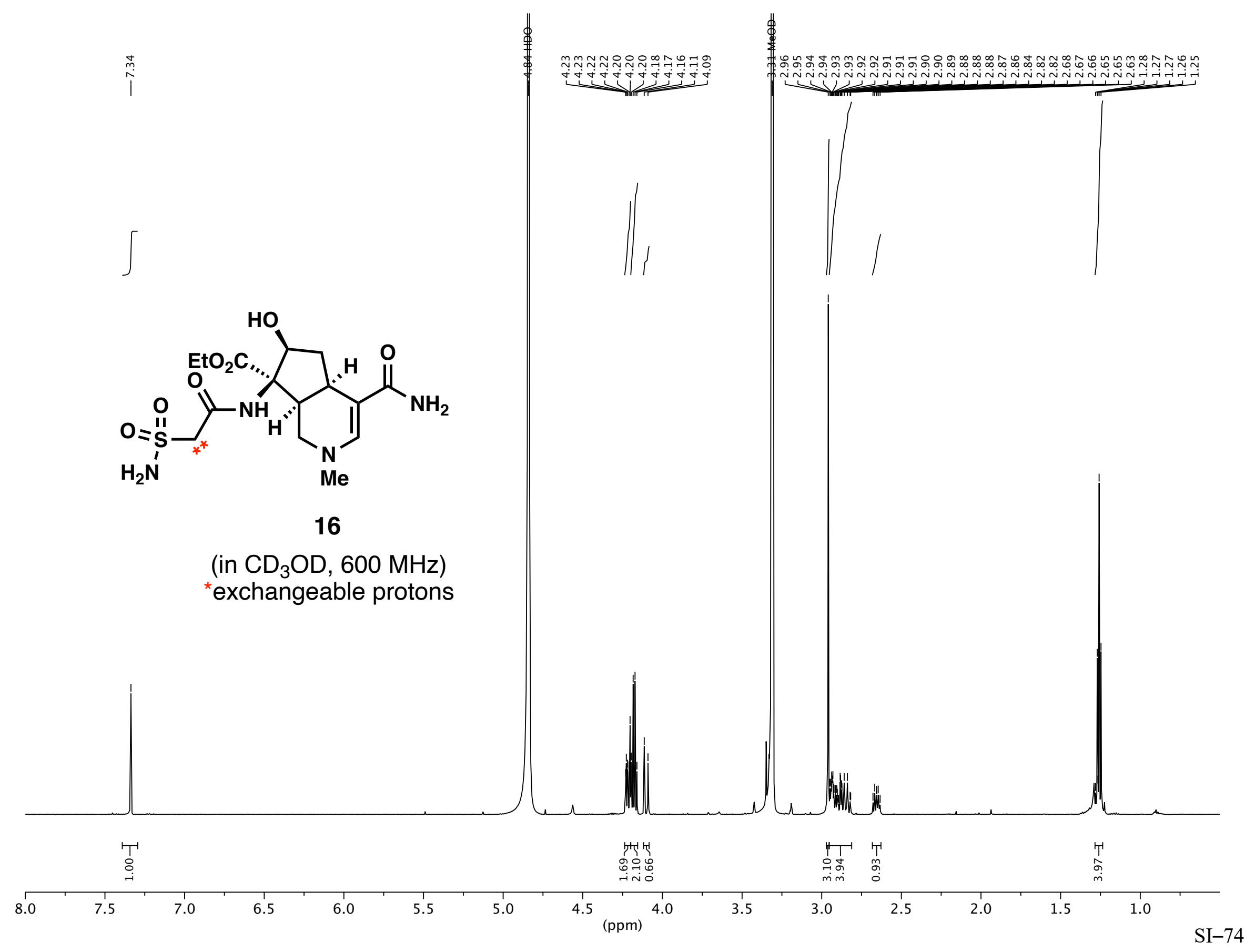




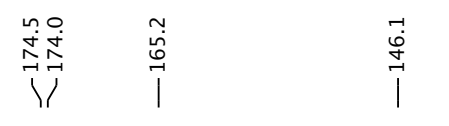

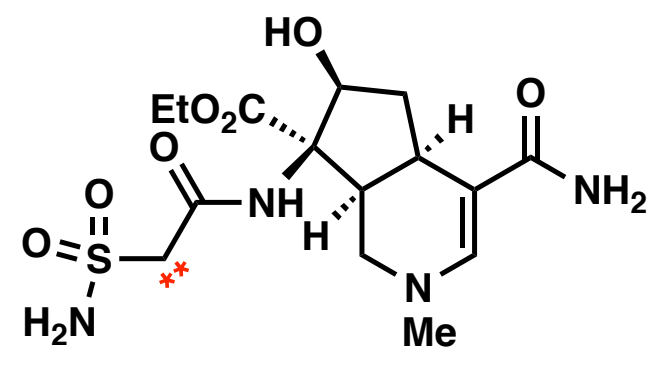

16

(in $\mathrm{CD}_{3} \mathrm{OD}, 151 \mathrm{MHz}$ )

*exchangeable protons

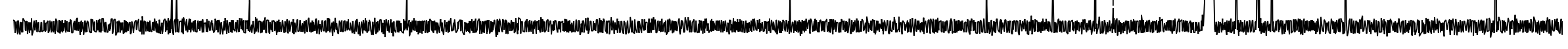

\begin{tabular}{|c|c|c|c|c|c|c|c|c|c|c|c|c|c|c|c|c|c|c|}
\hline 190 & 180 & 170 & 160 & 150 & 140 & 130 & 120 & 110 & $\begin{array}{c}100 \\
\mathrm{f} 1(\mathrm{ppm})\end{array}$ & 90 & 80 & 70 & 60 & 50 & 40 & 30 & 20 & $\begin{array}{r}10 \\
\text { SI-75 }\end{array}$ \\
\hline
\end{tabular}




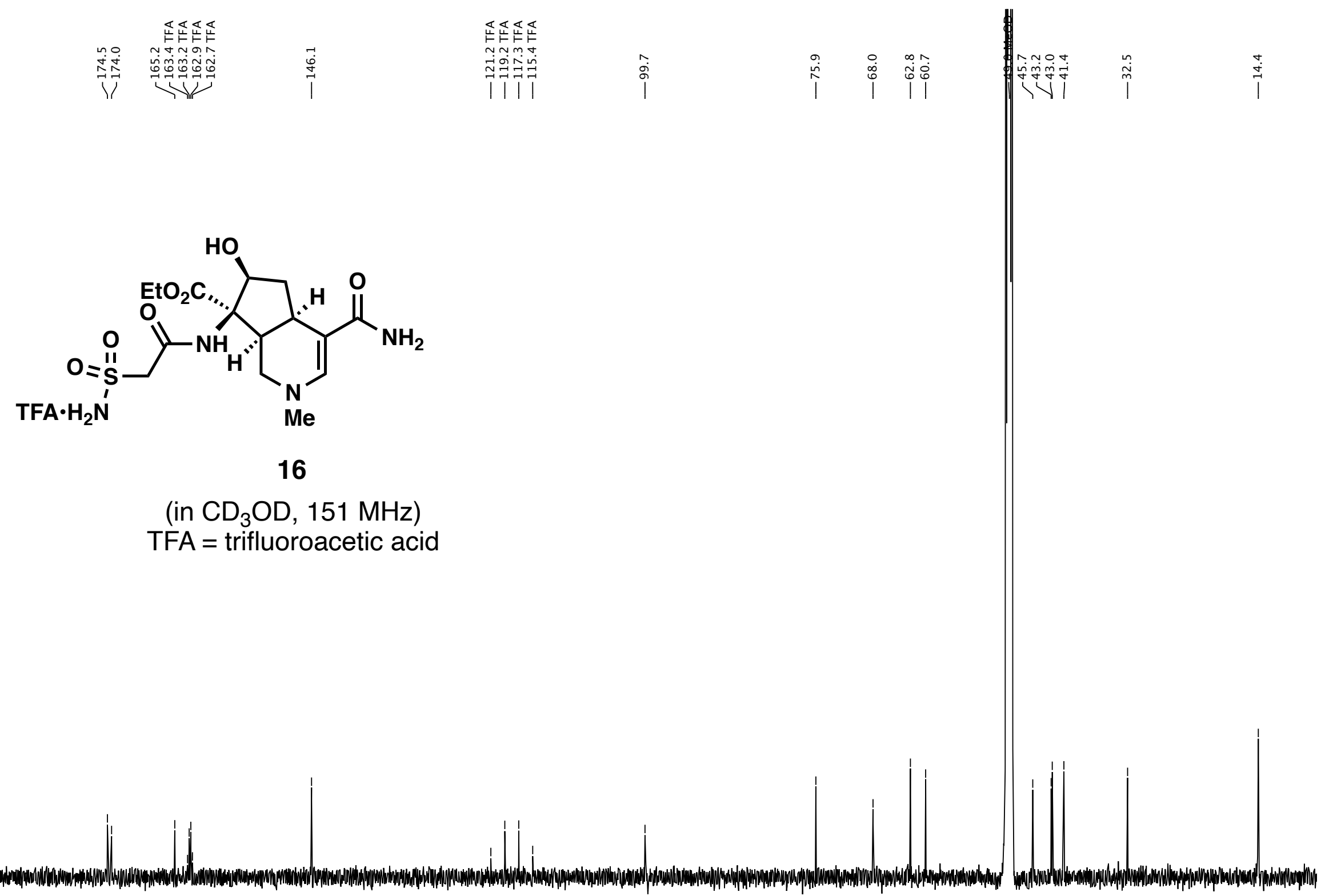

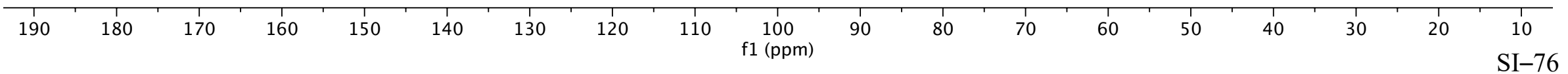




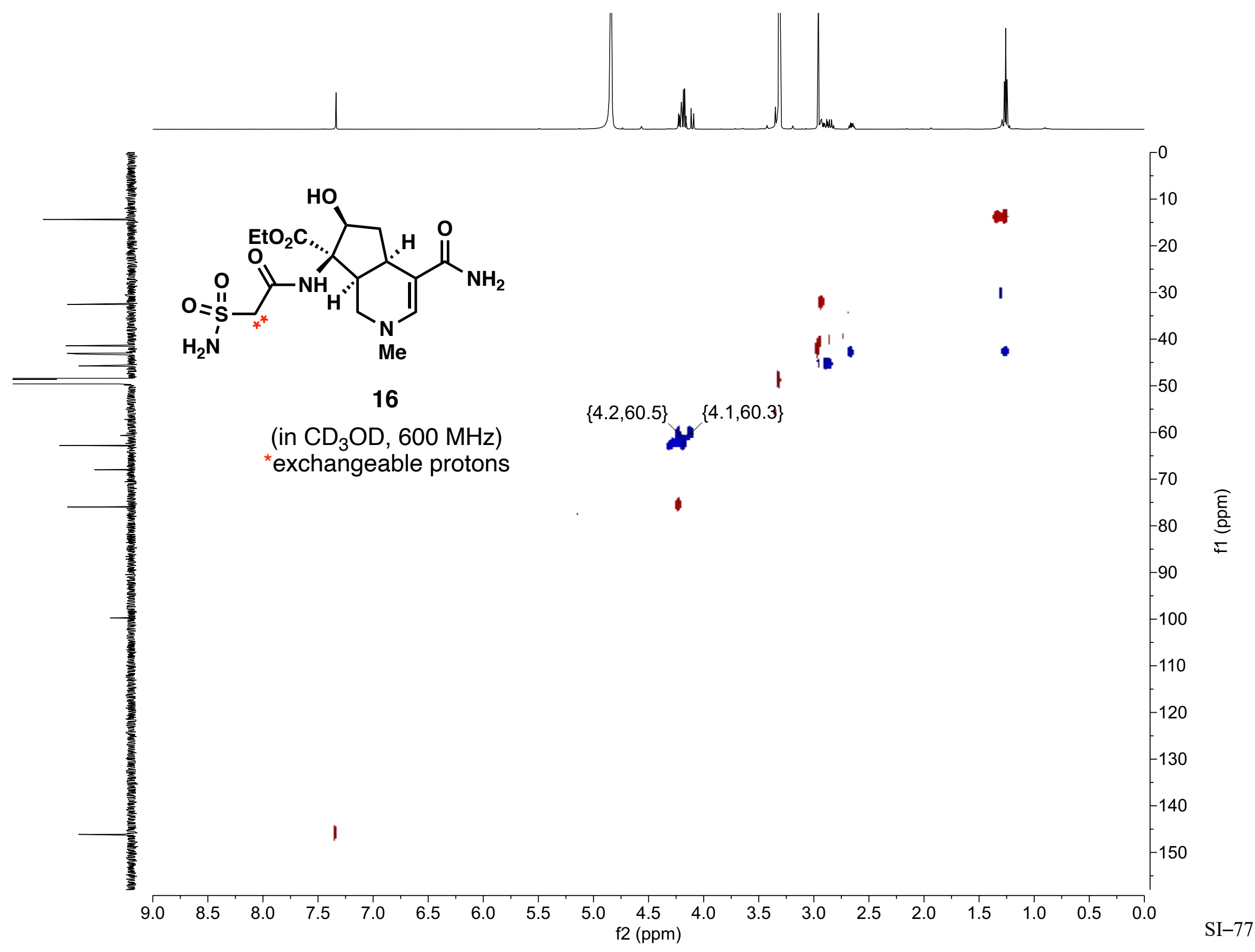




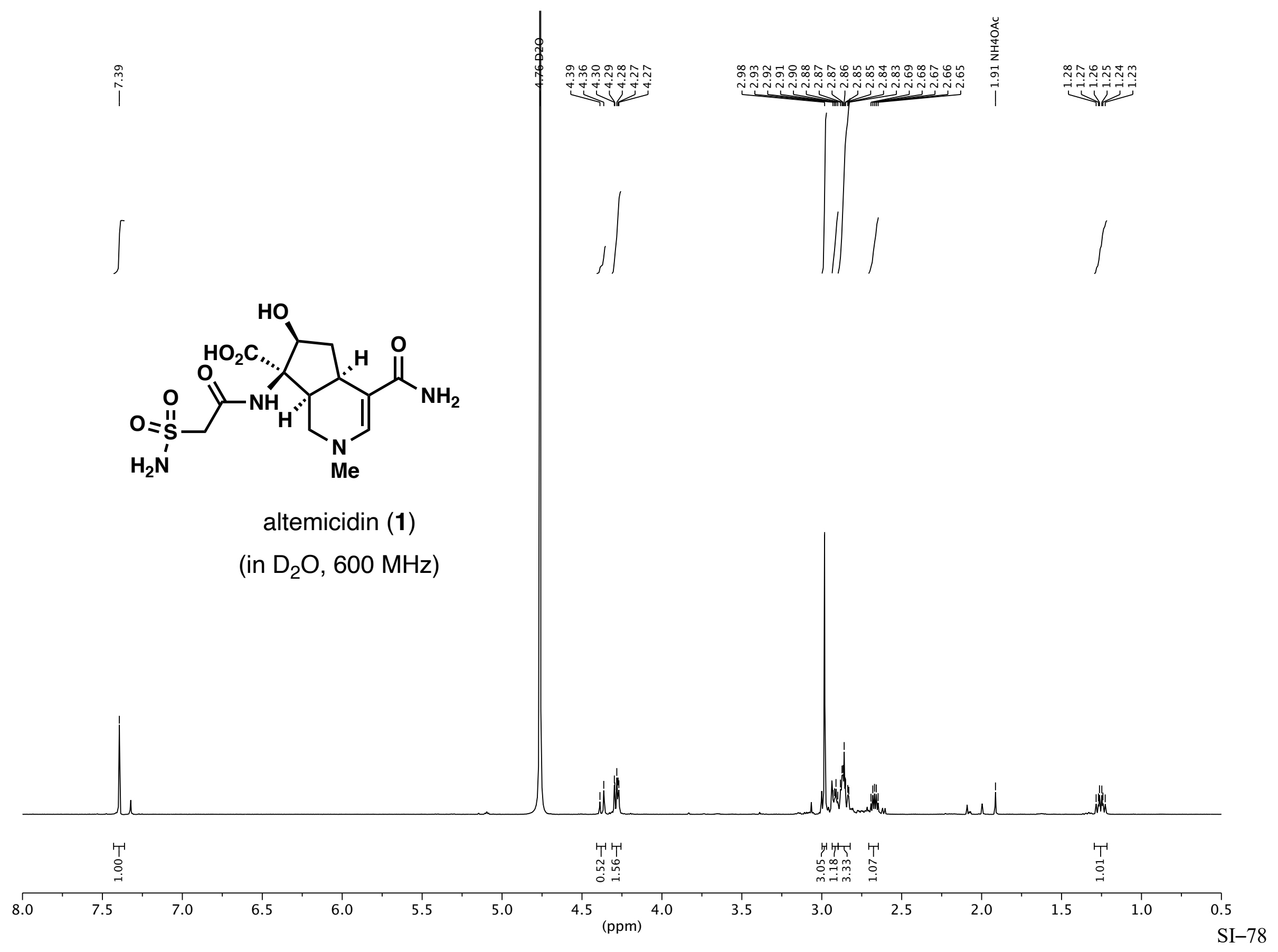




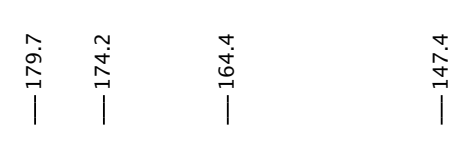

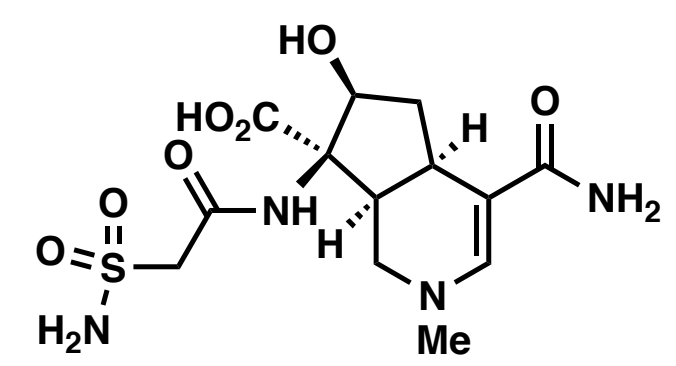

altemicidin (1)

(in $\mathrm{D}_{2} \mathrm{O}, 151 \mathrm{MHz}$ )

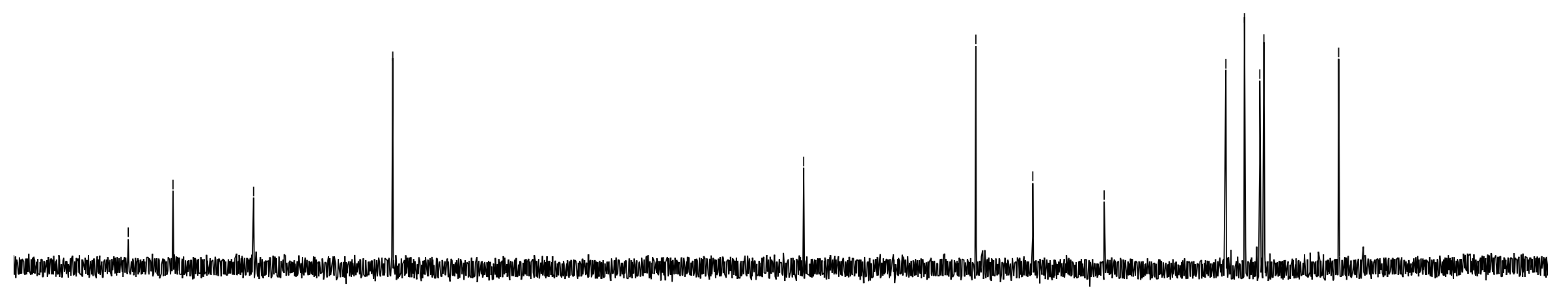

\begin{tabular}{|c|c|c|c|c|c|c|c|c|c|c|c|c|c|c|c|c|}
\hline 190 & 180 & 170 & 160 & 150 & 140 & 130 & 120 & 110 & $\begin{array}{c}100 \\
\text { f1 }(\mathrm{ppm})\end{array}$ & 90 & 80 & 70 & 60 & 50 & 40 & $\begin{array}{c}10 \\
\text { SI-79 }\end{array}$ \\
\hline
\end{tabular}


Synthetic $\left(\mathrm{D}_{2} \mathrm{O}, 151 \mathrm{mHz}\right.$, this work)

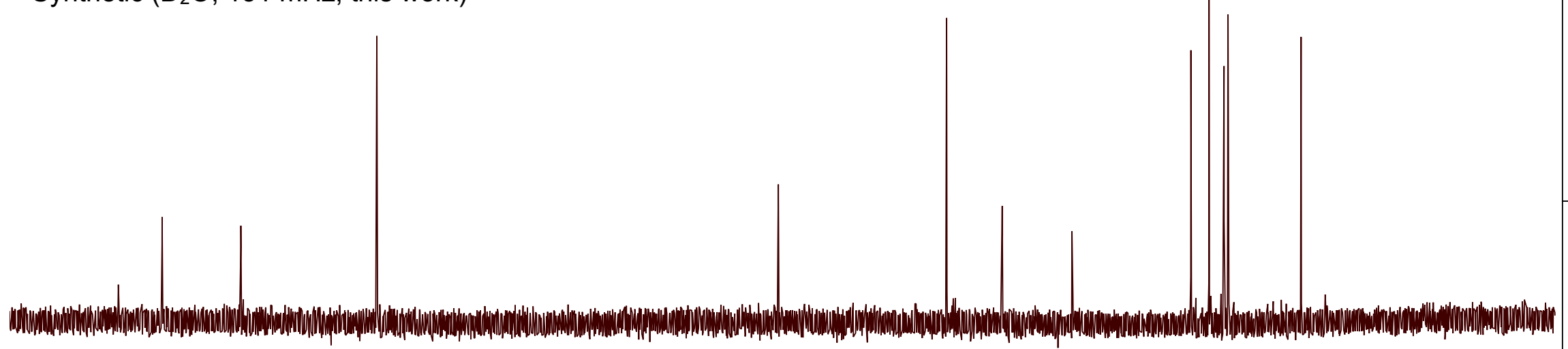

Authentic Sample $\left(\mathrm{D}_{2} \mathrm{O}, 226 \mathrm{mHz}\right.$, provide by Prof. Ikuro Abe, The University of Tokyo)

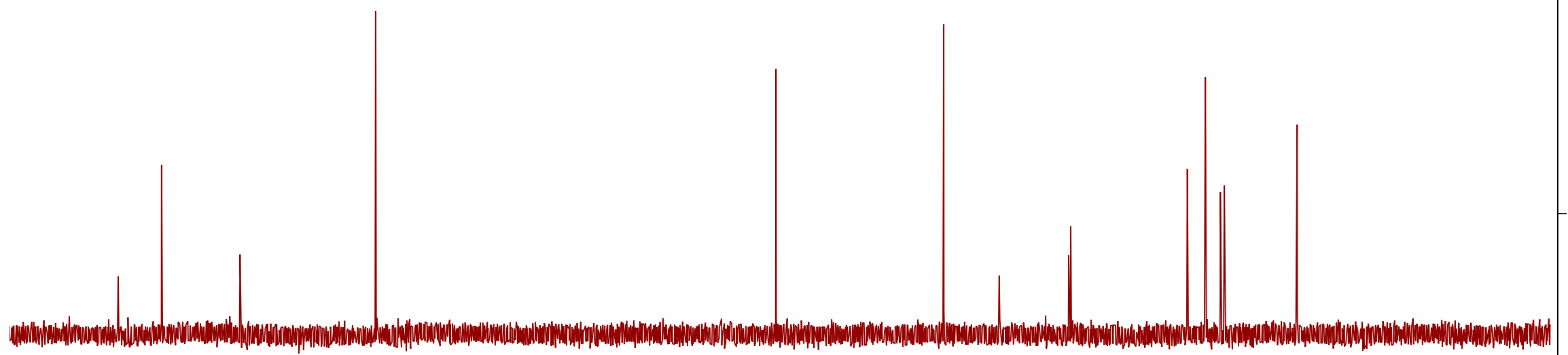




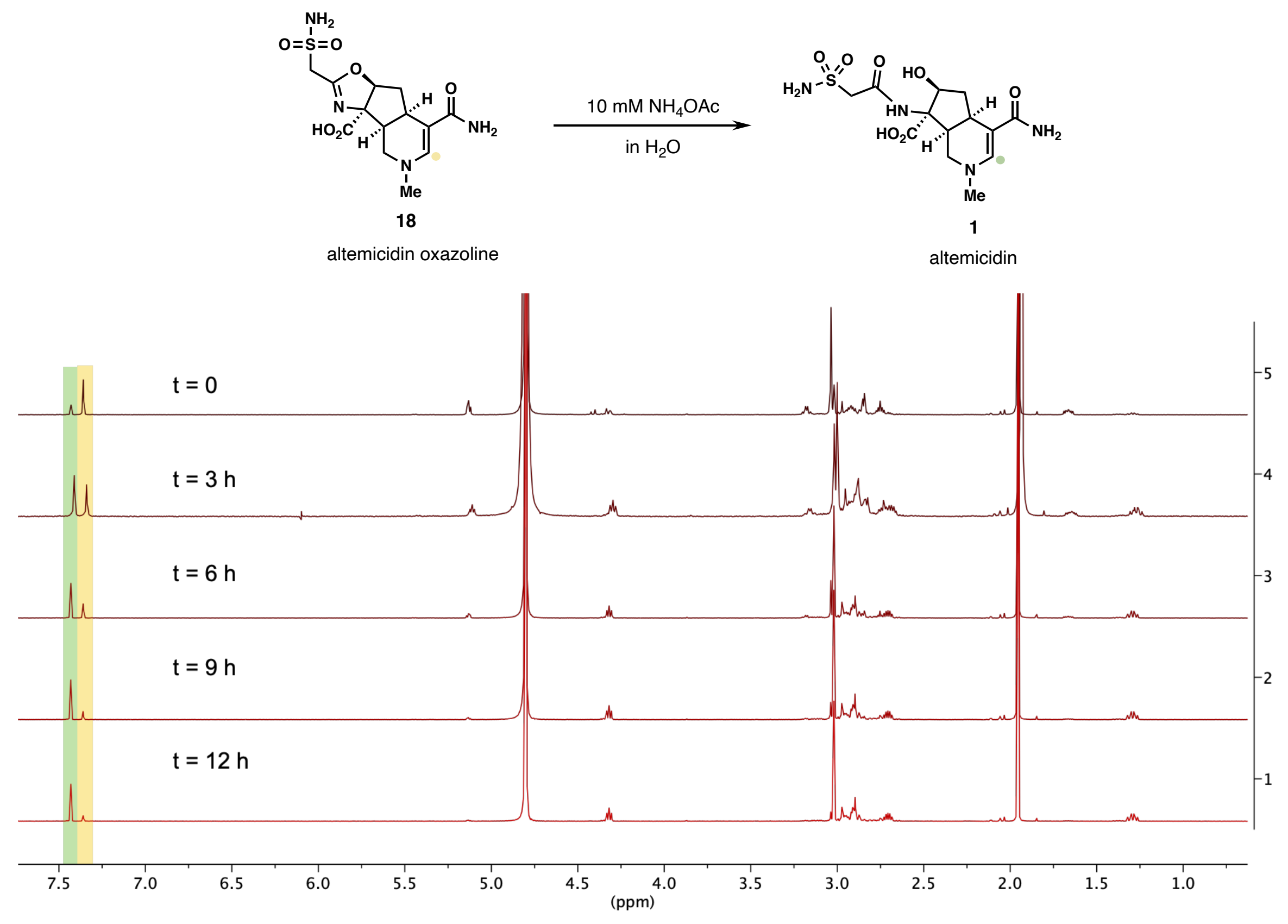




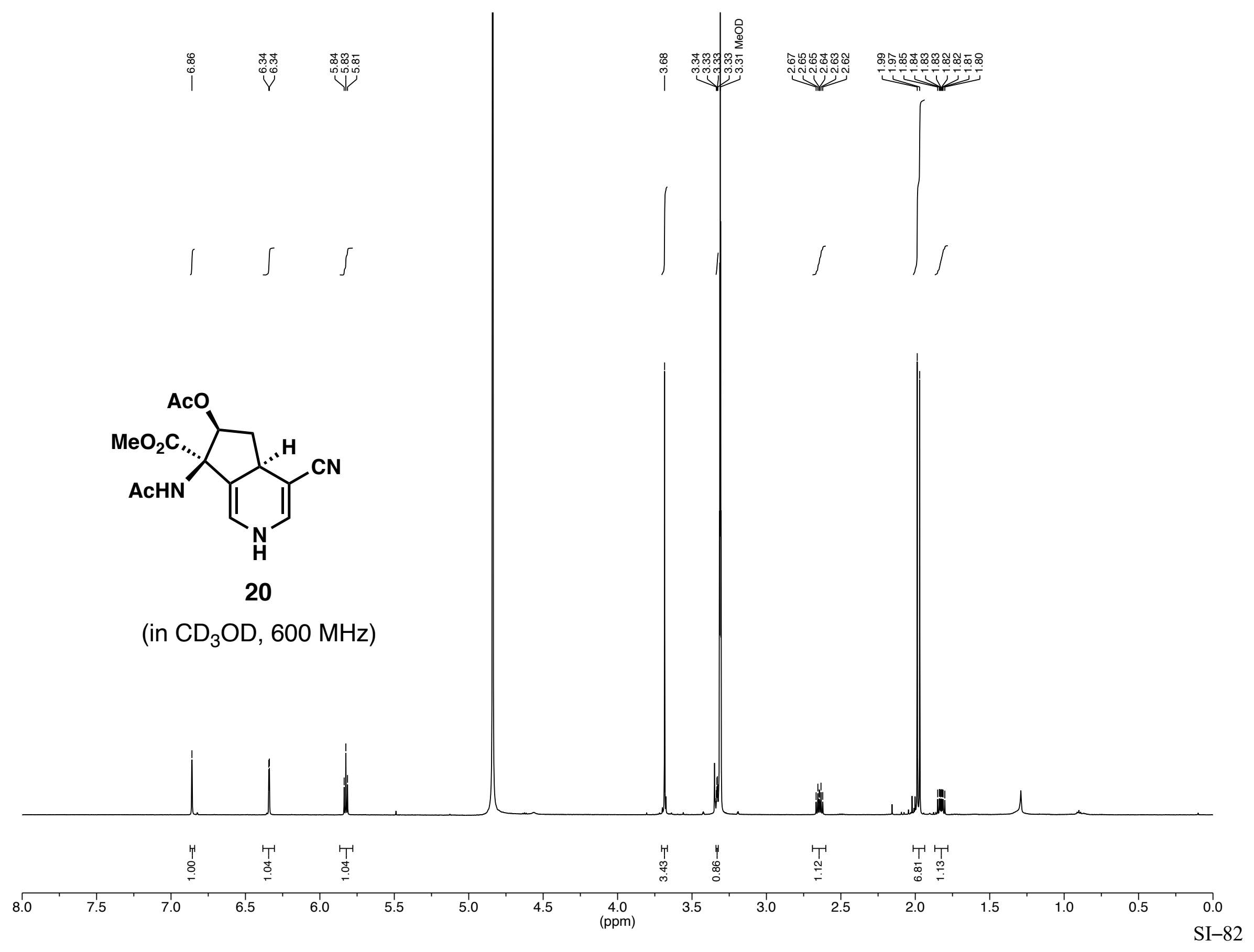




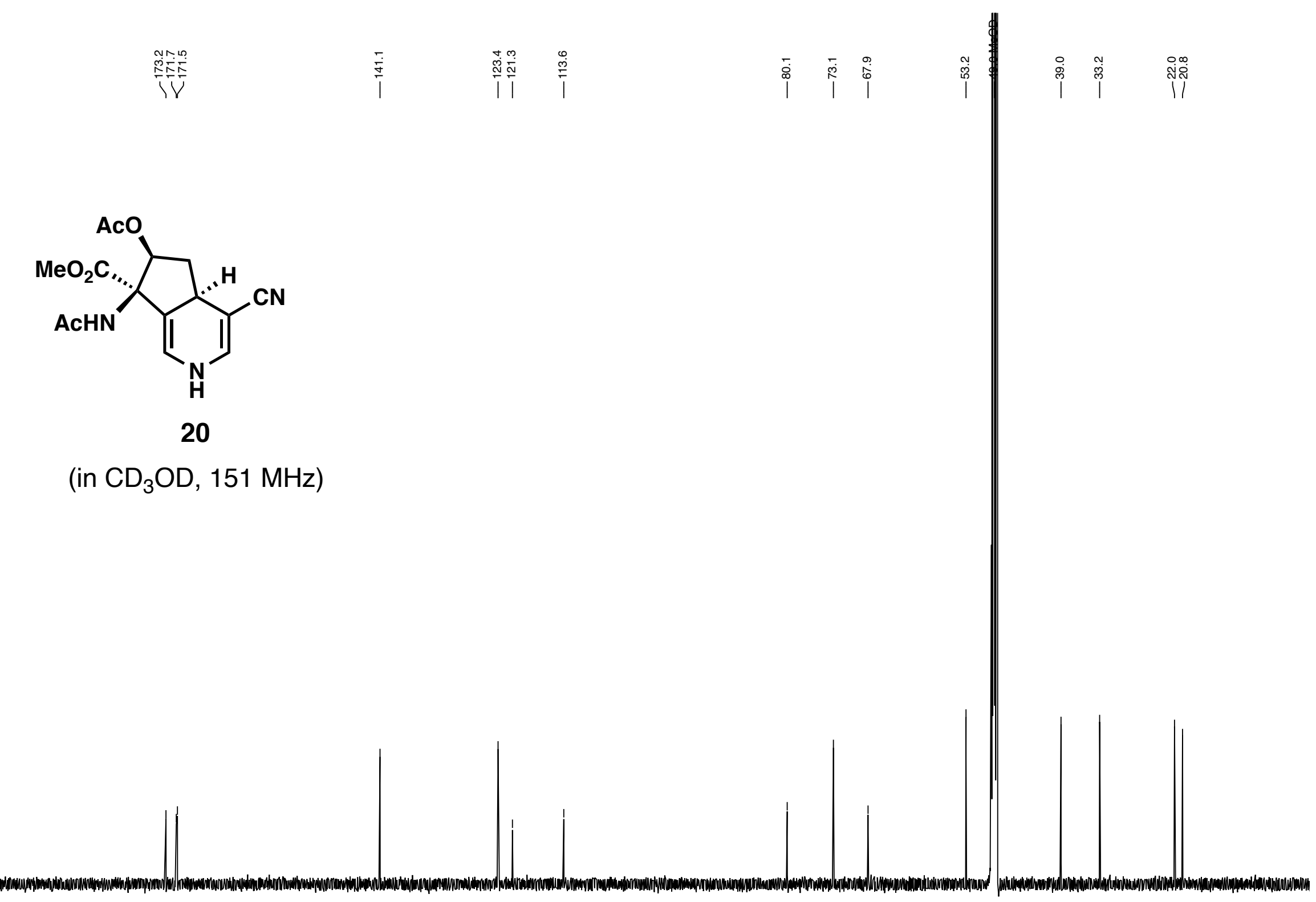




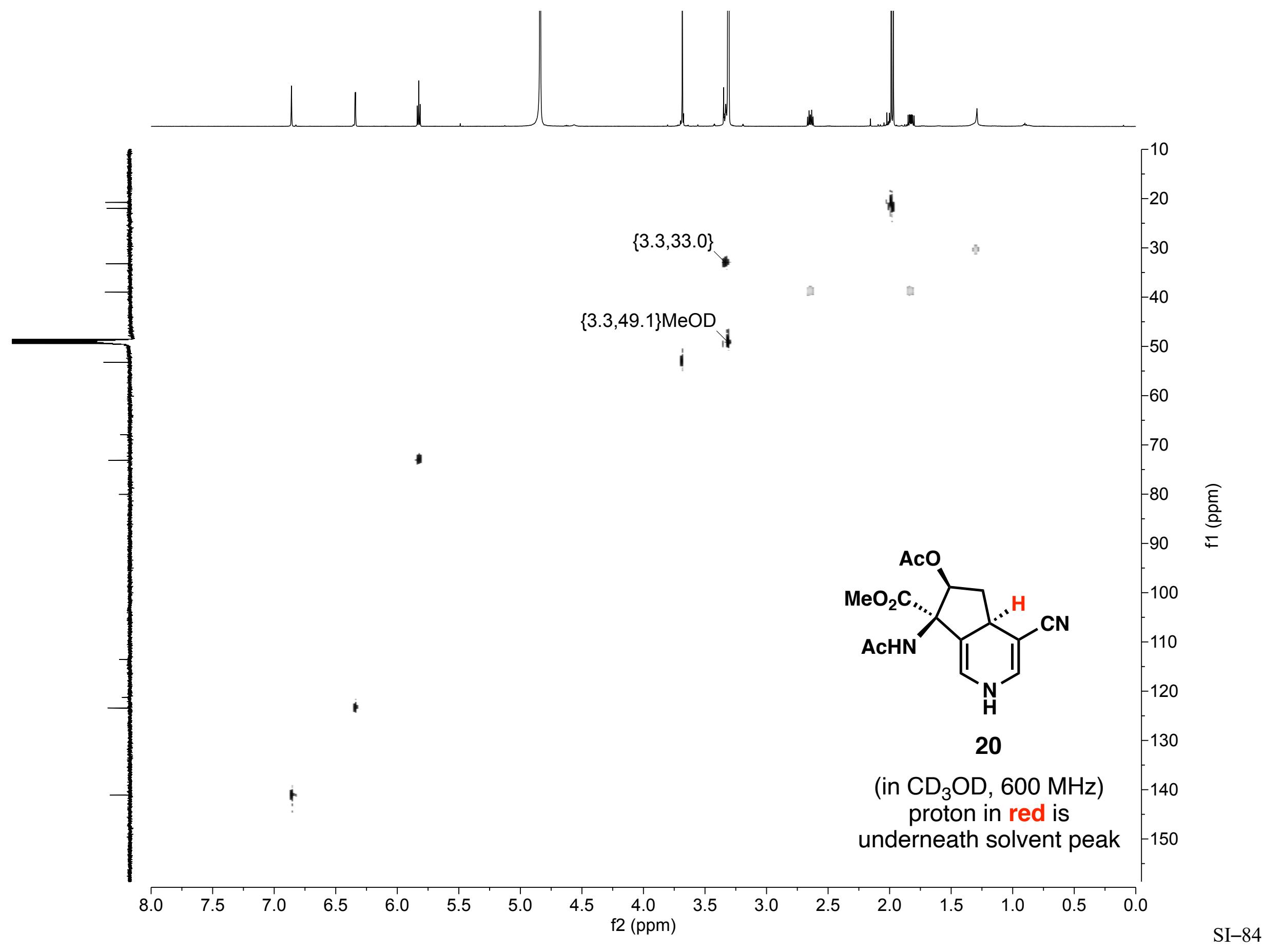




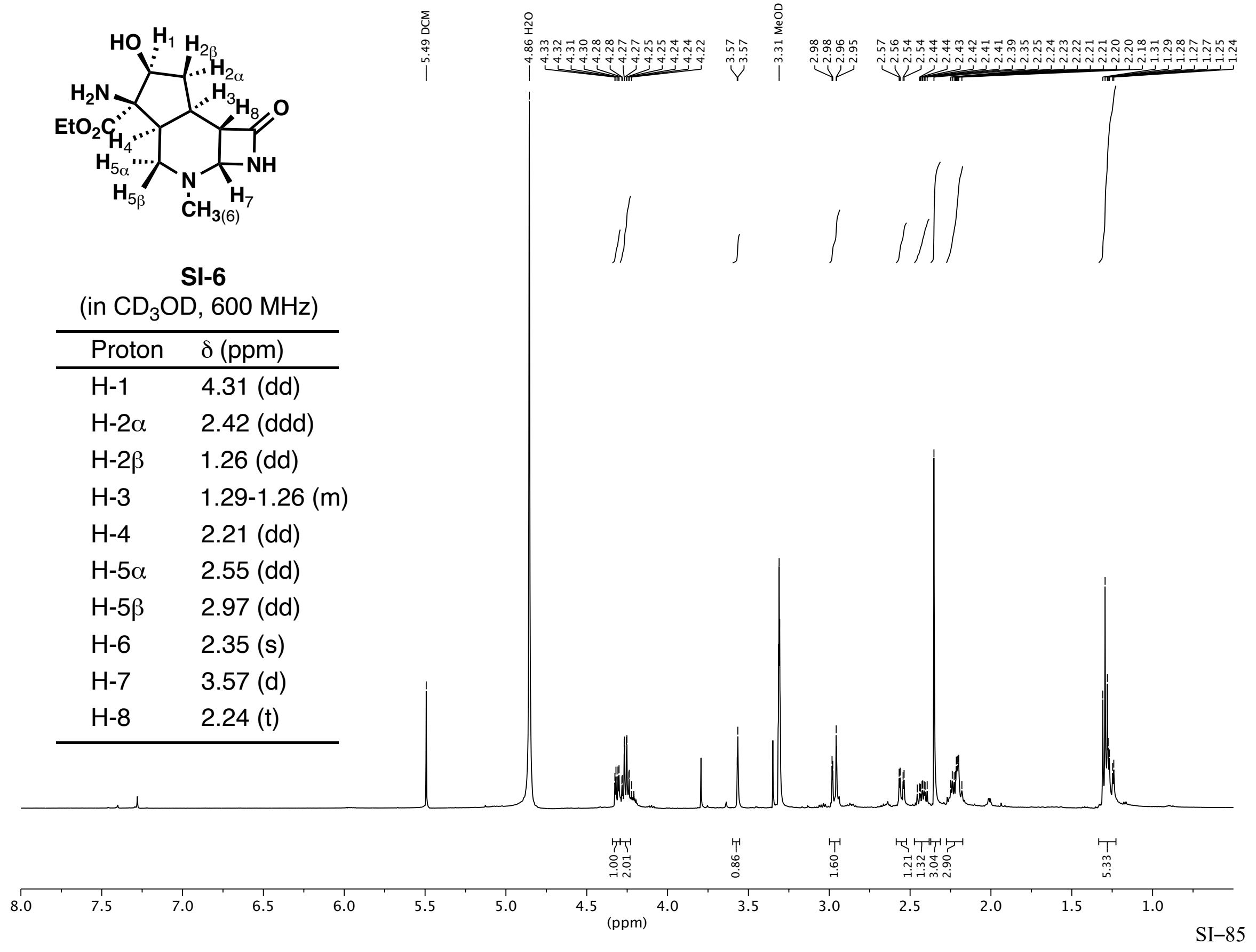




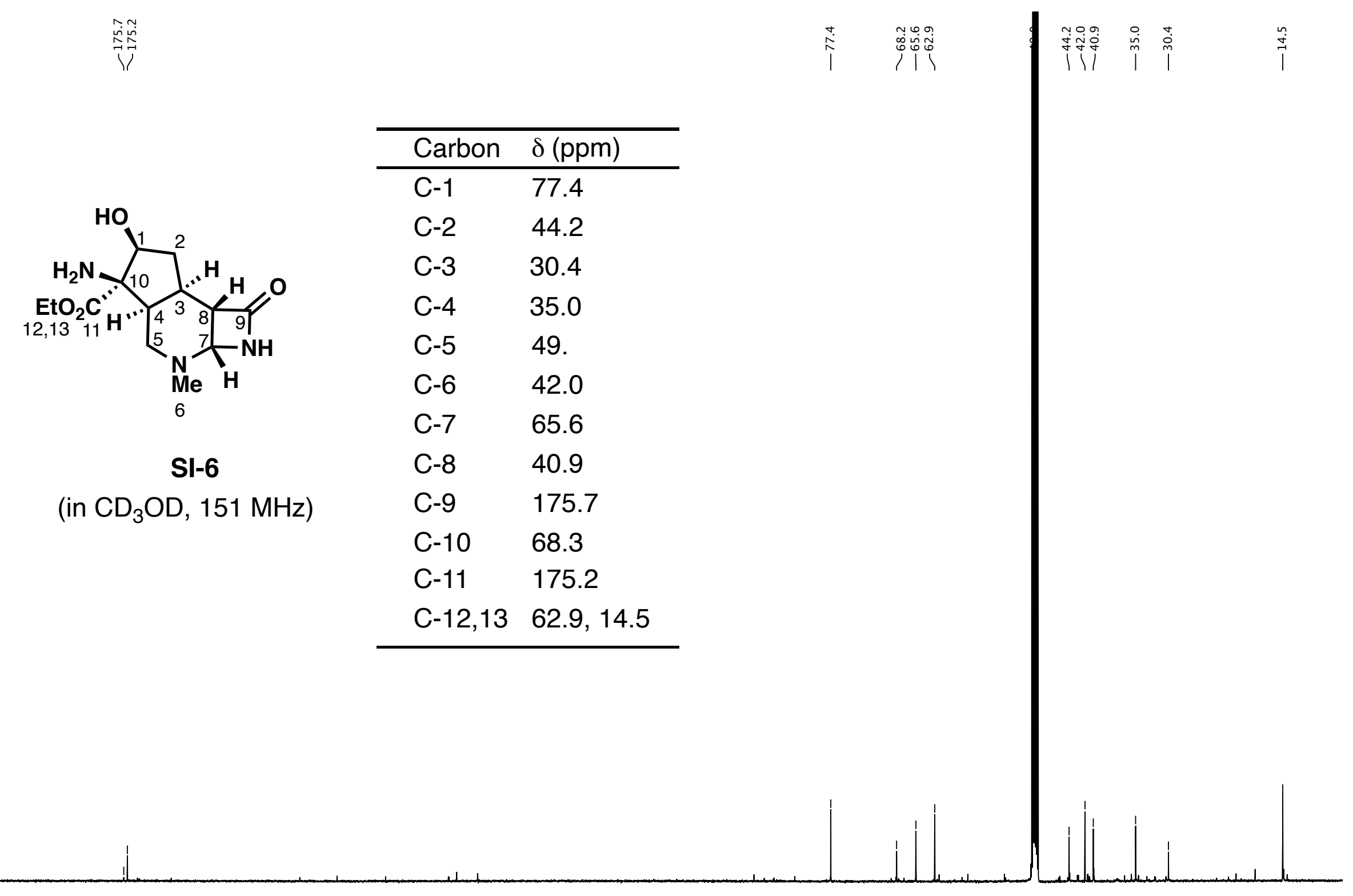

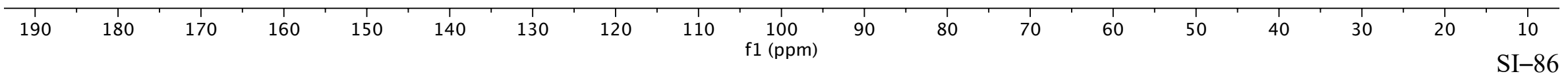




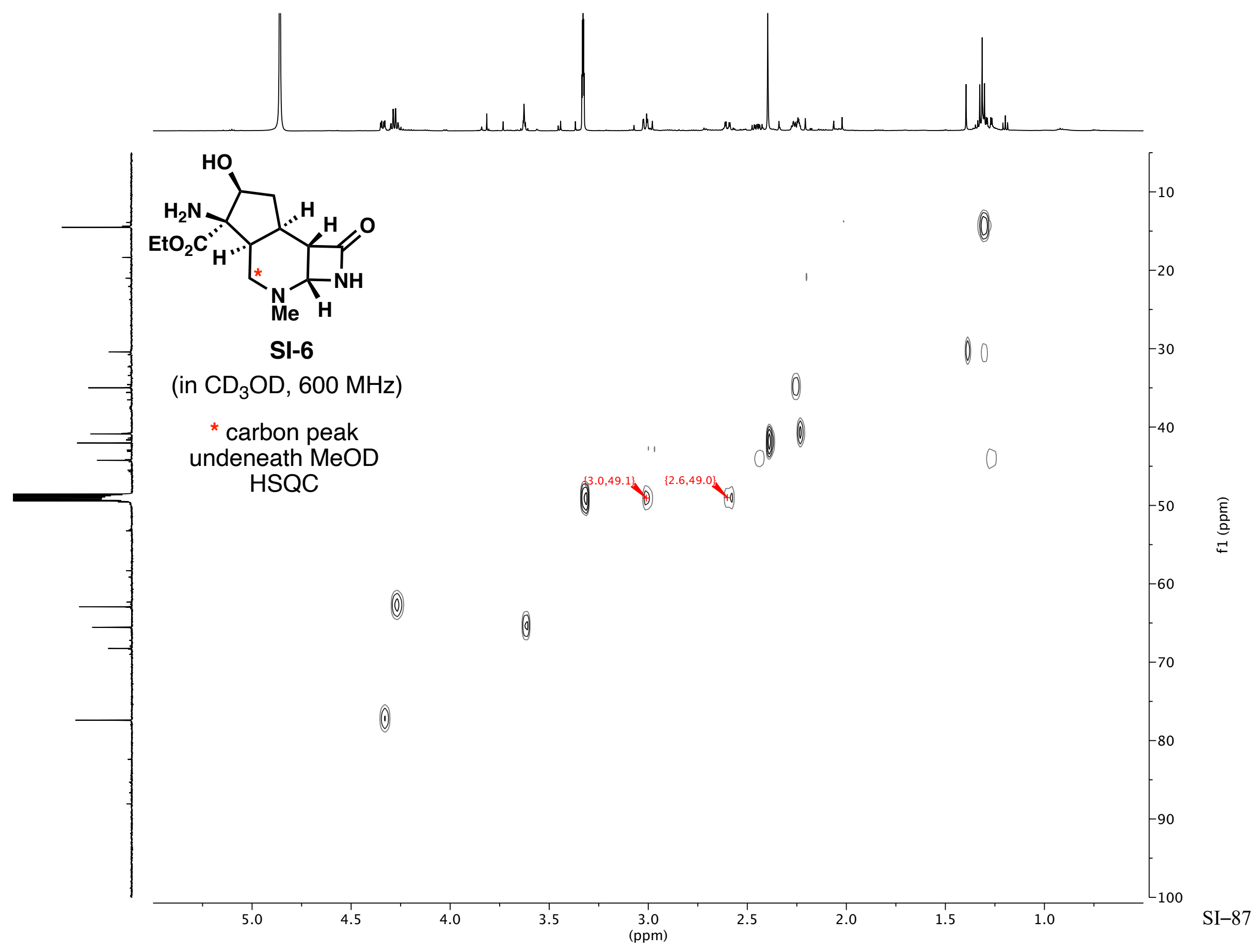




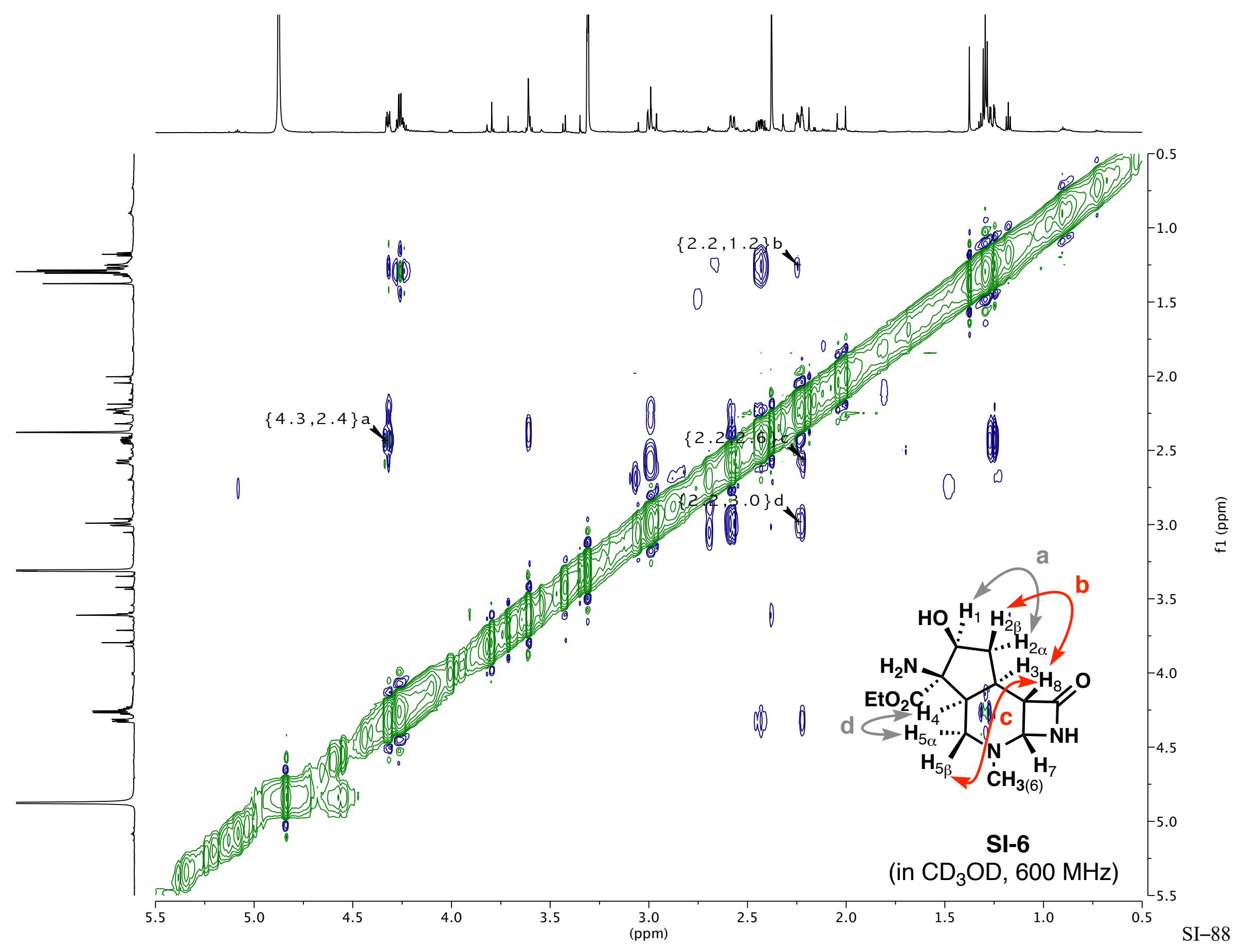




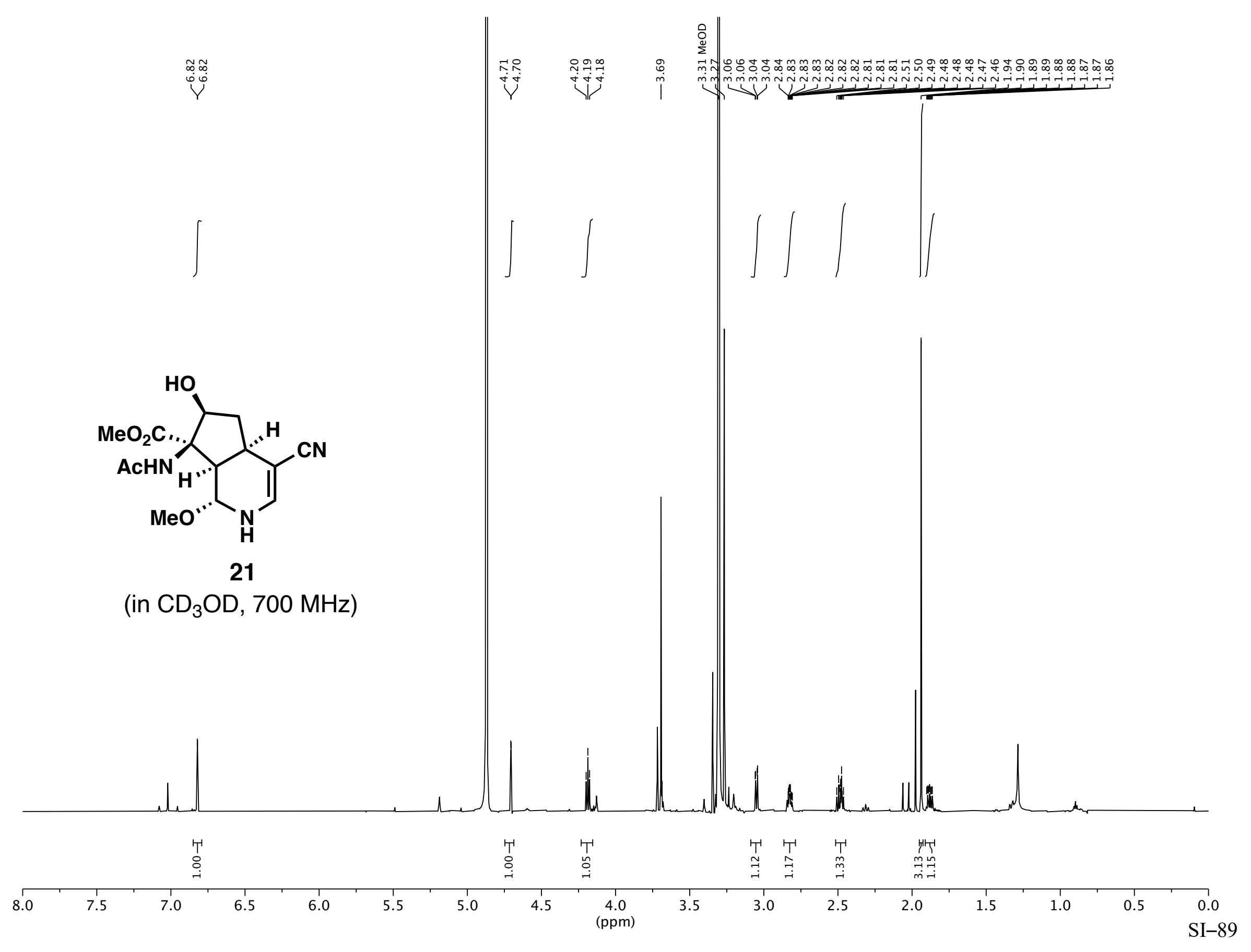




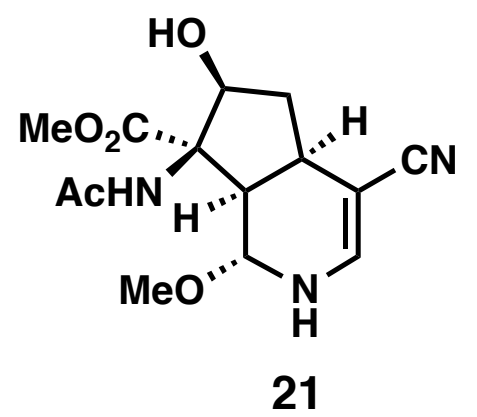

(in $\mathrm{CD}_{3} \mathrm{OD}, 151 \mathrm{MHz}$ )
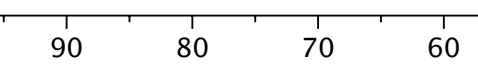

50 


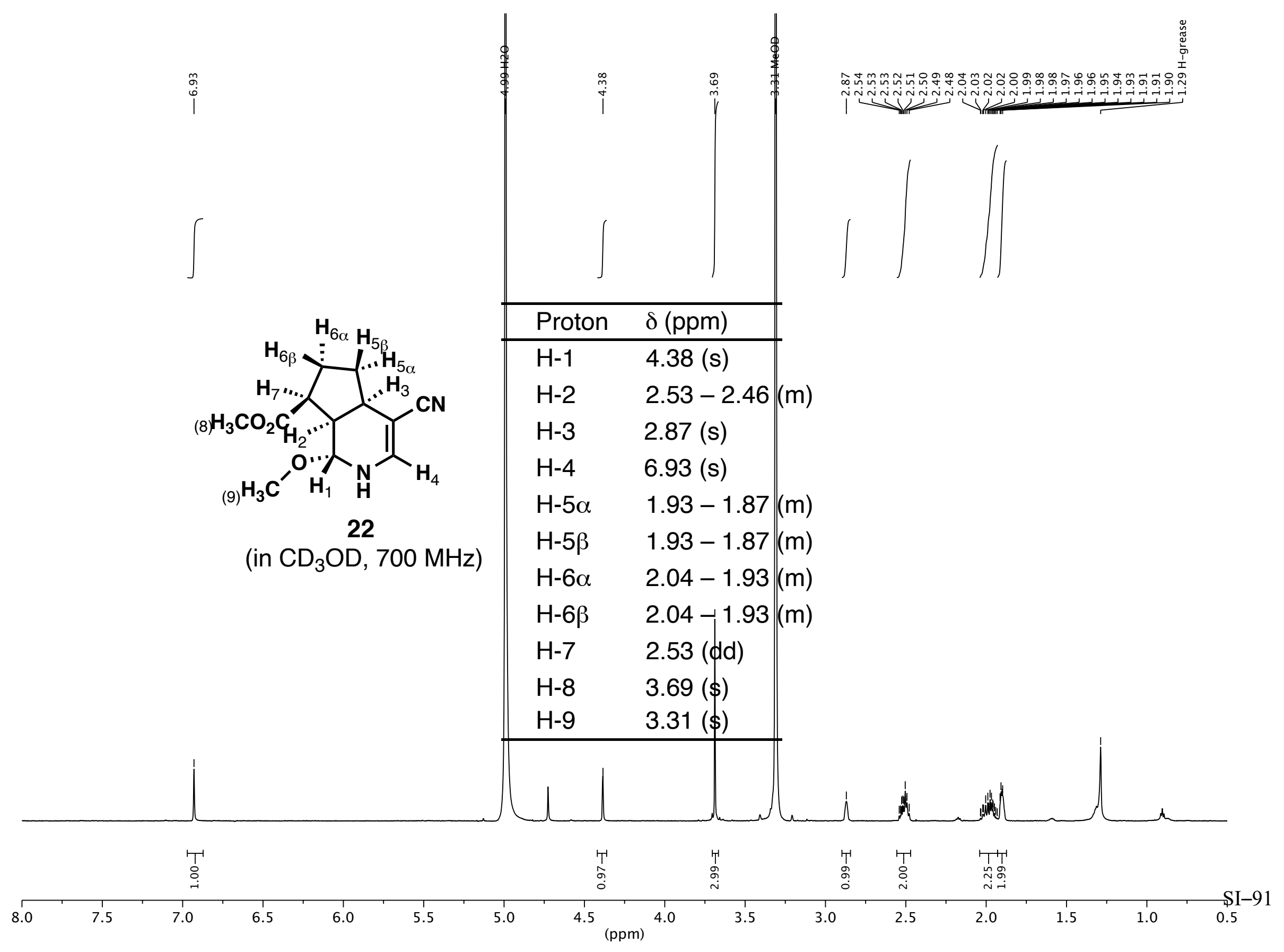




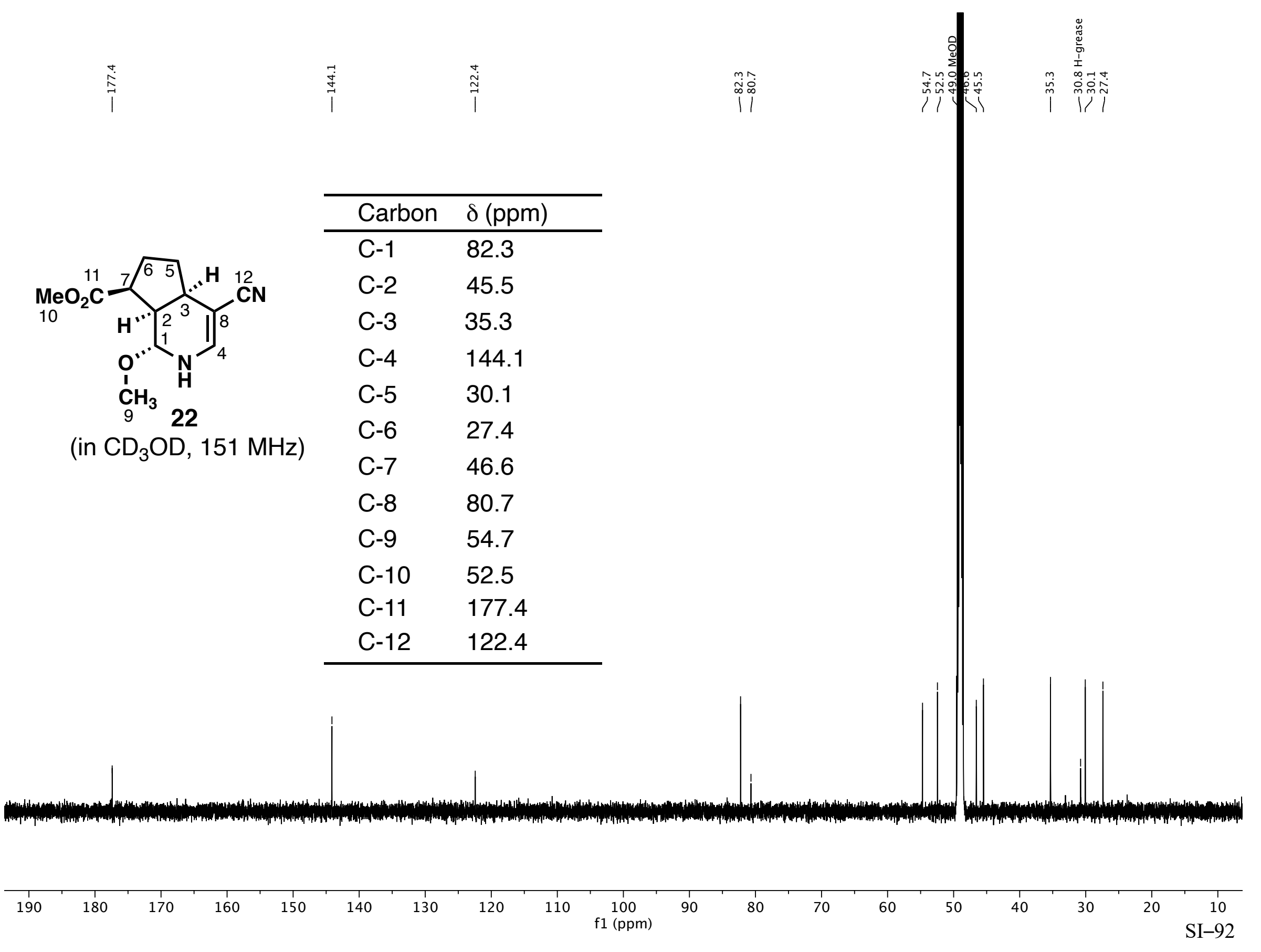




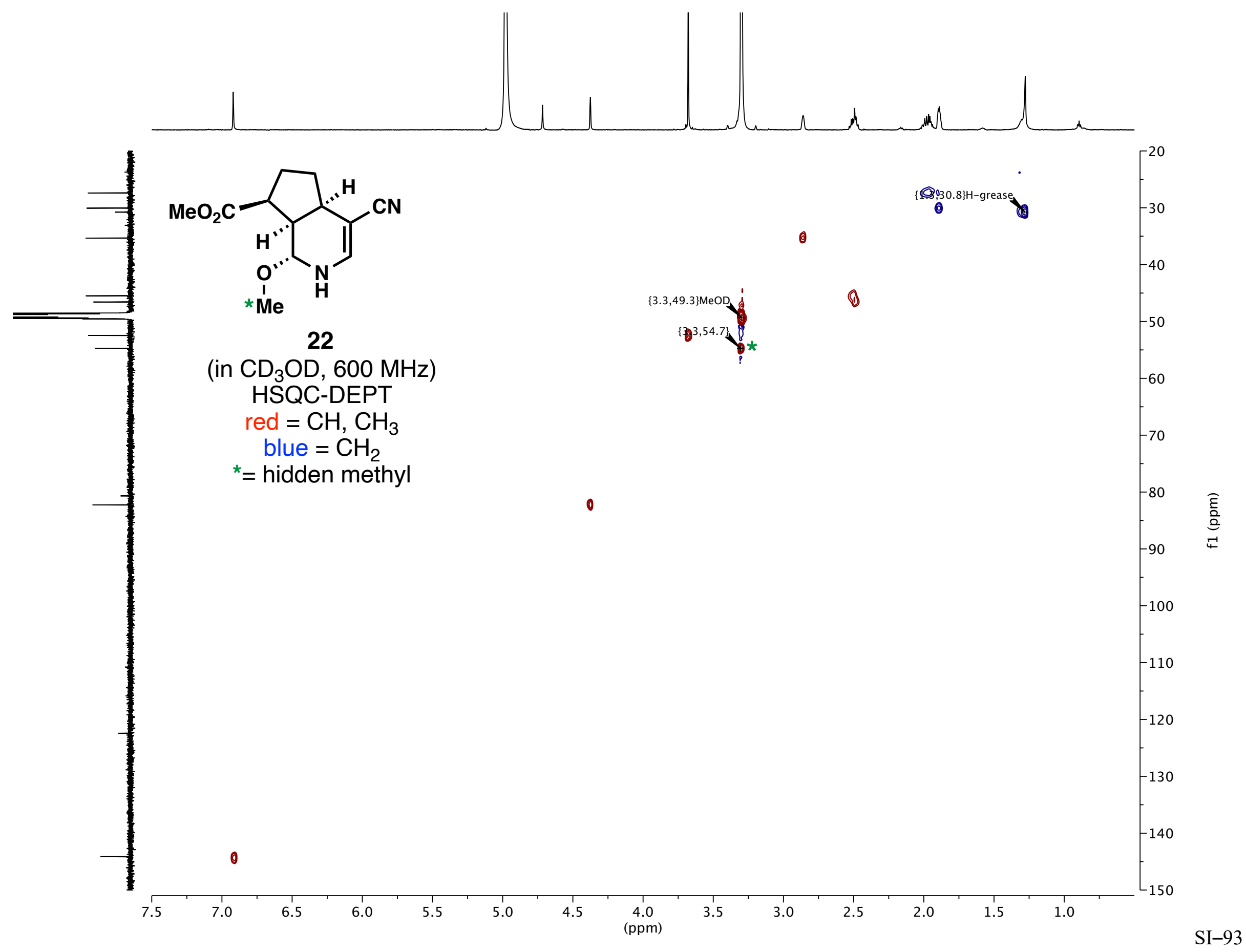




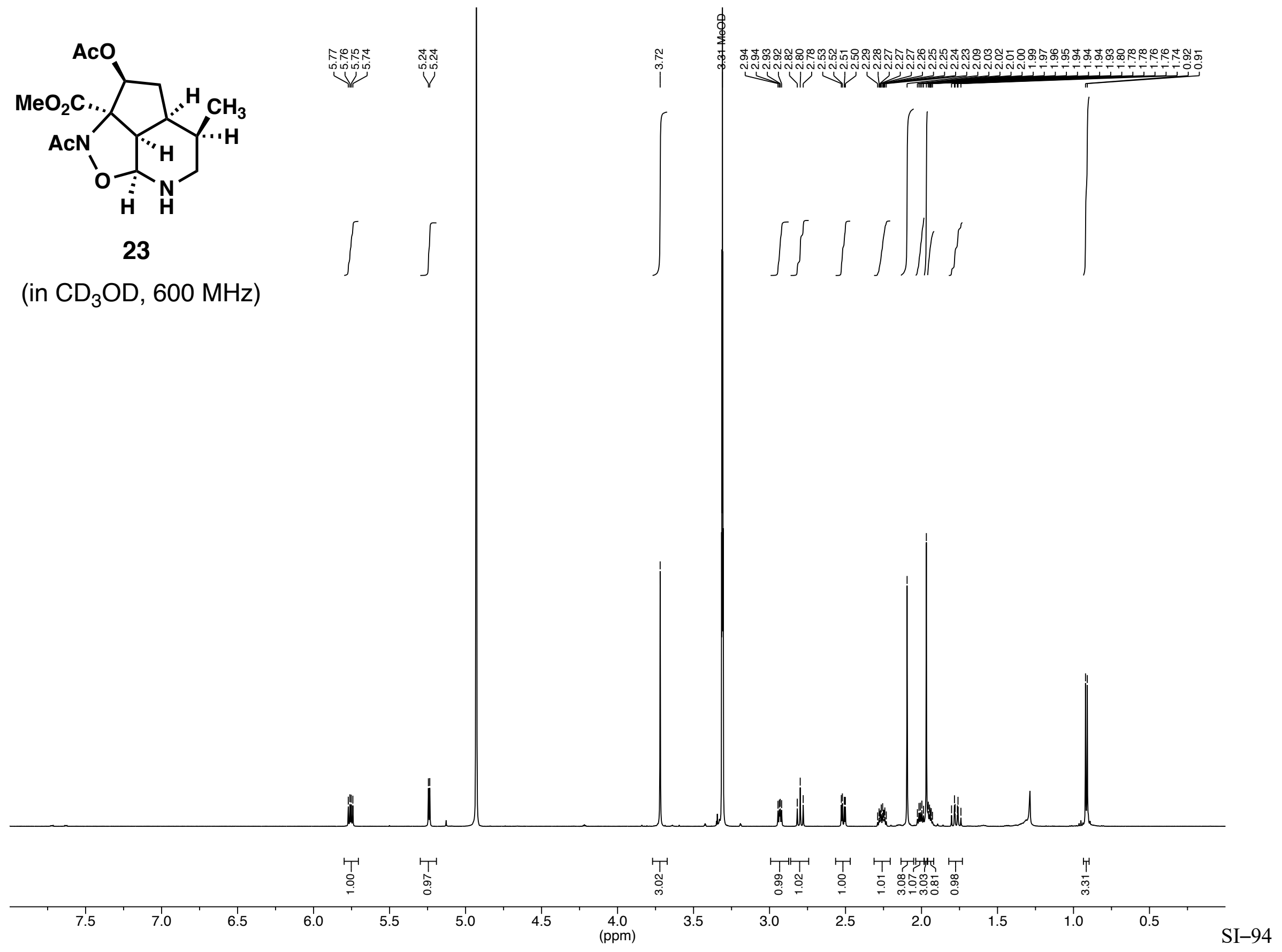




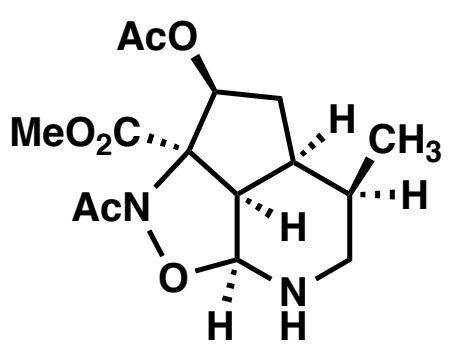

23

(in $\mathrm{CD}_{3} \mathrm{OD}, 151 \mathrm{MHz}$ )

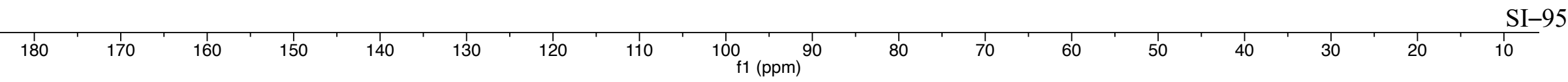




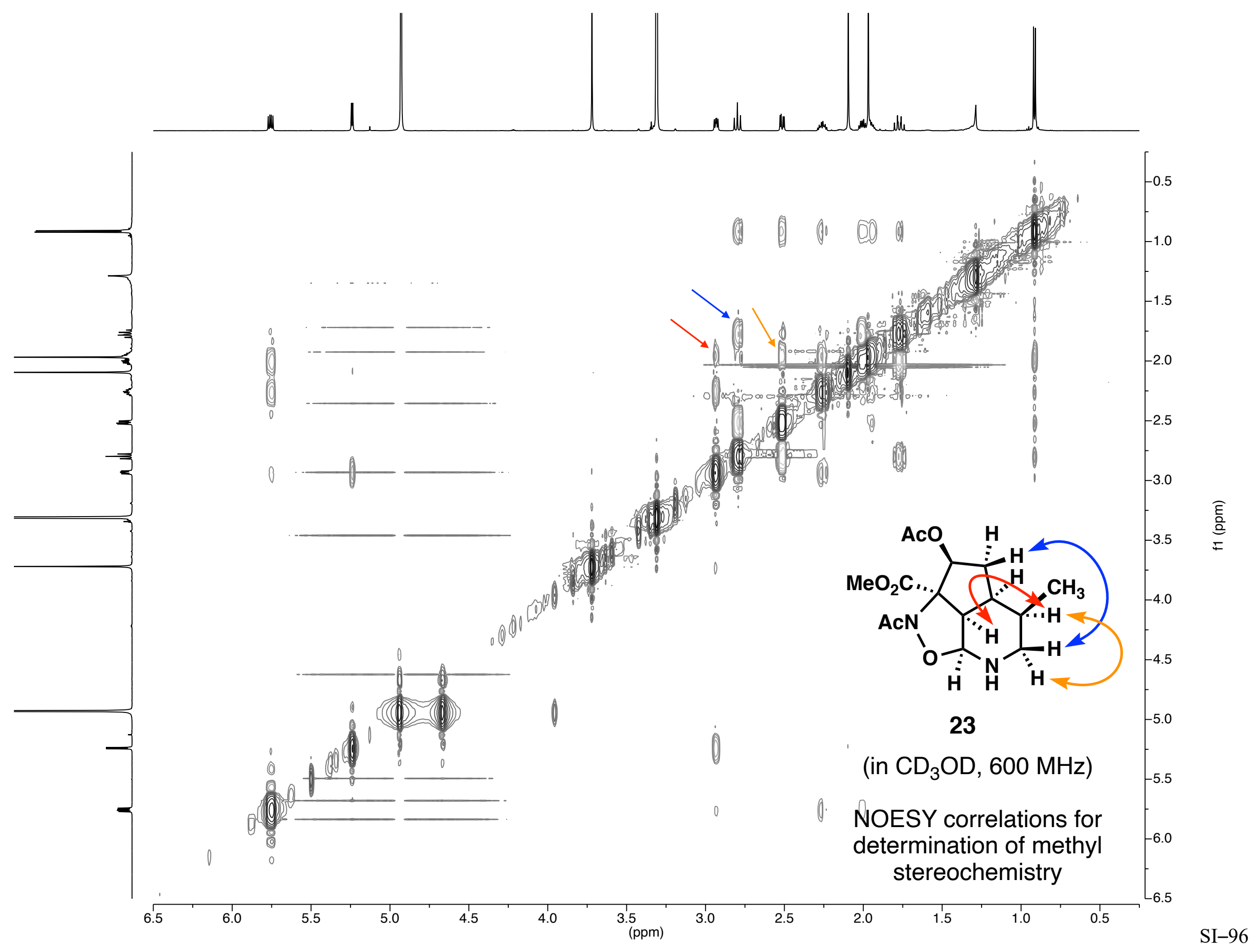

